Aus der Abteilung Klinische Chemie

(Prof.Dr.med.Dr.h.c. M.Oellerich) im Zentrum Innere Medizin

der Medizinischen Fakultät der Universität Göttingen

\title{
Die Pharmakokinetik von Meropenem bei Patienten mit schweren Infektionen
}

\author{
INAUGURAL - DISSERTATION \\ zur Erlangung des Doktorgrades \\ der Medizinischen Fakultät \\ der Georg-August-Universität zu Göttingen
}

vorgelegt von

Sebastian Hoppe

aus

Leinefelde

Göttingen 2010 
Dekan: Prof. Dr. med. C. Frömmel

I. Berichterstatter: Prof.Dr.med.Dr.h.c. Oellerich

II. Berichterstatter: Prof.Dr.med.Dr.rer.nat. Eiffert

III. Berichterstatterin: Prof.Dr.rer.nat. Virsik-Köpp

Tag der mündlichen Prüfung: 09.08.2010 


\section{Inhaltsverzeichnis}

Seite

$1 \quad$ Einleitung $\quad 7$

1.1 Antibiotikatherapie kritisch kranker Patienten 9

1.1.1 Spezielles Erregerspektrum und Resistenzlage $\quad 10$

1.1.2 Wirkkonzentrationen von Antibiotika am Ort der Infektion 11

1.1.3 Pharmakodynamik 14

1.1.4 Pharmakokinetik in Abhängigkeit von pathophysiologischen Einflüssen 15

$\begin{array}{lll}1.2 & \text { Die Eigenschaften von Meropenem } & 17\end{array}$

1.2.1 Antibakterielles Spektrum 17

$\begin{array}{lll}\text { 1.2.2 Pharmakokinetik von Meropenem } & 18\end{array}$

$2 \quad$ Patienten, Material und Methoden $\quad 24$

$2.1 \quad$ Ein- und Ausschlusskriterien der Patienten 24

2.2 Versuchsanordnung und Ablauf 24

2.3 Bestimmung der Antibiotikakonzentrationen mittels HPLC 25

2.4 Pharmakokinetische Parameter 27

2.5 Beurteilung der Schwere der Infektion 27

2.6 Beurteilung des Outcome der Patienten 32

$2.7 \quad$ Statistische Methoden 32

$3 \quad$ Ergebnisse 33

3.1 Demographische Daten 33

3.2 Hämatoonkologische Patienten 34

3.2.1 Demographische Daten der hämatoonkologischen Patienten 34

3.2.2 Diagnosen und weitere Erkrankungen der hämatoonkologischen Patienten 34

3.2.3 Laborparameter der hämatoonkologischen Patienten 34

3.2.4 Infektionsparameter der hämatoonkologischen Patienten 34

3.2.5 Pharmakokinetische Daten der hämatoonkologischen Patienten 36

3.2.6 Pharmakokinetische Daten der febrilen neutropenischen Patienten 38

3.2.7 Outcome der hämatoonkologischen Patienten 41 
$\begin{array}{lll}3.3 & \text { Intensivpatienten } & 43\end{array}$

3.3.1 Demographische Daten der Intensivpatienten 43

3.3.2 Laborparameter der Intensivpatienten 44

3.3.3 Infektionsparameter der Intensivpatienten 44

3.3.4 Pharmakokinetische Daten der Intensivpatienten 45

3.3.5 Pharmakokinetische Daten der Intensivpatienten in Abhängigkeit von der $\begin{array}{ll}\text { Nierenfunktion } & 47\end{array}$

3.3.6 Pharmakokinetische Daten der Intensivpatienten in Abhängigkeit von der Schwere der Infektion $\quad 51$

3.3.7 Outcome der Intensivpatienten $\quad 54$

$4 \quad$ Diskussion $\quad 56$

4.1 Therapieversagen unter Meropenem-Therapie 57

4.2 Unterschreitung erforderlicher Wirkspiegel von Meropenem 58

4.3 Ursachen der Unterschreitung erforderlicher Wirkspiegel von Meropenem 59

4.3.1 Hämatoonkologische Patienten 59

$\begin{array}{lll}\text { 4.3.2 Intensivpatienten } & 62\end{array}$

$\begin{array}{lll}4.4 & \text { Therapeutische Konsequenzen } & 68\end{array}$

$\begin{array}{lll}5 & \text { Zusammenfassung } & \mathbf{7 0}\end{array}$

$\begin{array}{lll}6 & \text { Literaturverzeichnis } & 72\end{array}$

$\begin{array}{lll}7 & \text { Abkürzungsverzeichnis } & 79\end{array}$

$8 \quad$ Anhang: Tabellen 7-25 $\quad 80$ 


\section{Verzeichnis der Abbildungen}

Abbildung 1: 2-Kompartment-Modell zur Beschreibung der Pharmakokinetik von Meropenem

Abbildung 2: Pathophysiologische oder iatrogene Konditionen mit Beeinflussung der Verteilung und Elimination von Antibiotika 16

Abbildung 3: Hämatoonkologische Patienten unterteilt nach Schwere der Infektion 35

Abbildung 4: Plasmakonzentrationsverläufe der hämotoonkologischen Patienten

Abbildung 5: Korrelation zwischen Kreatinin- und Meropenem-Clearance bei den hämatoonkologischen Patienten

Abbildung 6: Meropenem-Minimumkonzentration der hämatoonkologischen Patienten unterteilt in neutropenische Patienten (NP) und nicht-neutropenische Patienten (Nicht-NP)

Abbildung 7: Zeit-Konzentrations-Verlauf (semilog.) und Unterschreitung der Hemmkonzentrationen 2 und $4 \mathrm{mg} / 1$

Abbildung 8: Anzahl der hämatoonkologischen Patienten mit verschiedenen Schweregraden der Infektion unterteilt in nicht-neutropenische und neutropenische Patienten

Abbildung 9: Outcome der hämatoonkologischen Patienten

Abbildung 10: Unterschiede der wichtigsten pharmakokinetischen Parameter zwischen neutropenischen (NP) und nicht-neutropenischen (Nicht-NP) hämatoonkologischen Patienten

Abbildung 11: Intensivpatienten unterteilt nach Schwere der Infektion

Abbildung 12: Zusammenhang zwischen Kreatinin-Clearance und MeropenemClearance bei Intensivpatienten ohne CVVHF oder Z.n.CVVHF

Abbildung 13: Plasmakonzentrationsverläufe der Intensivpatienten in Abhängigkeit von der Dosis und dem Ausmaß der Nierenfunktionseinschränkung

Abbildung 14: Meropenem-Clearance der Intensivpatienten unterteilt nach dem Ausmaß der Nierenfunktionseinschränkung

Abbildung 15: Verteilungsvolumen bei hämatoonkologischen Patienten, Intensivpatienten mit Sepsis und Intensivpatienten mit septischem Schock

Abbildung 16: Anteil der Patienten mit und ohne Nierenfunktionseinschränkung unter den Patienten mit Sepsis und septischem Schock

Abbildung 17: Meropenem-Clearance der Intensivpatienten unterteilt nach Sepsis und septischem Schock

Abbildung 18: Outcome der Intensivpatienten 


\section{Verzeichnis der Tabellen}

Tabelle 1: Outcome-Klassifikation unter Meropenem-Therapie 32

Tabelle 2: Demographische Daten 33

Tabelle 3: Demographische Daten der hämatoonkologischen Patienten 34

Tabelle 4: Schweregrad der Infektion bei hämatoonkologischen Patienten 35

Tabelle 5: Demographische Daten der Intensivpatienten 43

Tabelle 6: Unterteilung der Intensivpatienten nach dem Schweregrad der Infektion 44

Tabelle 7: Diagnosen und weitere relevante Erkrankungen der hämatoonkologischen $\begin{array}{ll}\text { Patienten } & 80\end{array}$

Tabelle 8: Laborparameter der hämatoonkologischen Patienten 81

Tabelle 9: Infektionsparameter der hämatoonkologischen Patienten 82

Tabelle 10: Pharmakokinetische Parameter der hämatoonkologischen Patienten Teil 183

Tabelle 11: Pharmakokinetische Parameter der hämatoonkologischen Patienten Teil 284

Tabelle 12: Pharmakokinetische Parameter der hämatoonkologischen Patienten unterteilt in Neutropeniker und Nicht-Neutropeniker Teil 1

Tabelle 13: Pharmakokinetische Parameter der hämatoonkologischen Patienten unterteilt in Neutropeniker und Nicht-Neutropeniker Teil 2

Tabelle 14: Diagnosen und weitere relevante Erkrankungen der Intensivpatienten Teil1 87

Tabelle 15: Diagnosen und weitere relevante Erkrankungen der Intensivpatienten Teil2 88

Tabelle 16: Laborparameter der Intensivpatienten 89

Tabelle 17: Infektionsparameter der Intensivpatienten Teil 1

Tabelle 18: Infektionsparameter der Intensivpatienten Teil 2

Tabelle 19: Schweregrad der Infektion der Intensivpatienten 92

Tabelle 20: Pharmakokinetische Parameter der Intensivpatienten Teil 1

Tabelle 21: Pharmakokinetische Parameter der Intensivpatienten Teil 2

Tabelle 22: Pharmakokinetische Parameter der Intensivpatienten ohne Nierenfunktionseinschränkung Teil 1

Tabelle 23: Pharmakokinetische Parameter der Intensivpatienten ohne Nierenfunktionseinschränkung Teil 2

Tabelle 24: Pharmakokinetische Parameter der Intensivpatienten mit Nierenfunktionseinschränkung Teil $1 \quad 97$

Tabelle 25: Pharmakokinetische Parameter der Intensivpatienten mit Nierenfunktionseinschränkung Teil 2 


\section{$1 \quad$ Einleitung}

Das Carbapenem-Antibiotikum Meropenem ist gekennzeichnet durch sein breites antibakterielles Spektrum und seine gute Verträglichkeit. Im klinischen Alltag findet es unter anderem Anwendung zur empirischen Therapie schwerer Infektionen bei Intensivpatienten und bei febrilen hämatoonkologischen Patienten. Infektionen haben in diesen Patientengruppen eine hohe Inzidenz und sind eine der häufigsten Todesursachen. Die Anwendung von Meropenem-,,Standarddosierungen“ führt jedoch bei diesen kritisch kranken Patienten häufig trotz nachgewiesener Empfindlichkeit der Keime zum Therapieversagen. Die Ursachen dieses pharmakodynamischen Therapieversagens bei Patienten mit schweren Infektionen und die resultierenden therapeutischen Konsequenzen waren Inhalt der vorliegenden Studie.

Neben der grundsätzlichen Wirksamkeit eines Antibiotikums, also der Sensibilität des zu bekämpfenden Erregers, sind ausreichend hohe Konzentrationen des Therapeutikums am Ort der Infektion zwingende Voraussetzung einer erfolgreichen antiinfektiösen Behandlung.

Die meisten Antibiotika zeigen eine dosisabhängige Wirkung und können ohne Zuhilfenahme von Plasmaspiegelbestimmungen körpergewichtsbezogen dosiert werden. Dosis, Wirkspiegel und therapeutischer Effekt zeigen eine gute Korrelation. Die therapeutische Breite des Medikamentes ist ausreichend, so dass ein verminderter therapeutischer Effekt aufgrund Unterdosierung sowie ein unerwünschter toxischer Effekt durch Überdosierung vermieden werden kann. Krankheitsbedingte Veränderungen der Pharmakokinetik des verwendeten Antibiotikums können jedoch die Dosis-Wirkungsbeziehung abschwächen oder gar aufheben.

Zahlreiche Untersuchungen zur antibiotischen Therapie bei Intensivpatienten haben gezeigt, dass die Ergebnisse gesunder Probanden oder leicht erkrankter Patienten nicht ohne weiteres auf kritisch kranke Patienten übertragen werden dürfen (Gomez et al. 1999, Joukhadar et al. 2001). Schwere Infektionen, Sepsis und septischer Schock führen häufig zu Veränderungen der pharmakokinetischen und pharmakodynamischen Parameter verschiedener Medikamente (Boucher et al. 2006). Ihre Wirkung unterliegt somit einer interindividuellen Variabilität. Bei einigen Medikamenten, wie z.B. Vasopressoren, ist die Wirkung direkt am klinischen Erfolg abzulesen, was eine Individualisierung der Dosierung ermöglicht. Um auch bei den kausal wirkenden Antibiotika, deren Therapieerfolg häufig erst anhand von Fieberverlauf, klinischer Symptomatik, bildgebenden Verfahren oder Akutphaseproteinen nach einem Zeitintervall von einigen Tagen abgeschätzt werden kann, trotz aller Variabilität eine effektive Therapie zu gewährleisten und Resistenzentwicklungen zu vermeiden, sind Untersuchungen zur Dosisindividualisierung auch in dieser Patientengruppe erforderlich. 
Da immer weniger neue Antibiotika mit neuen Wirkmechanismen auf den Markt kommen und gleichzeitig eine Zunahme der Resistenzentwicklung bestimmter Keime beobachtet wird, ist eine Optimierung der Therapie mit den vorhandenen Medikamenten entscheidend.

Das Carbapenem-Antibiotikum Meropenem ist seit vielen Jahren insbesondere bei der Therapie schwerer Infektionen mit unbekannten Erregern in klinischem Gebrauch. Es wird üblicherweise ohne Drug Monitoring eingesetzt, da publizierte Studien eine gute Korrelation zwischen Dosis und therapeutischem Effekt zeigen konnten. Die überwiegende Anzahl der veröffentlichten Untersuchungen bezieht sich jedoch auf Patienten mit leichten oder mittelschweren Infektionen. Studien mit Risikopatienten, bei denen eingreifende Veränderungen der Dosis-Konzentrations-Wirkungsrelation zu erwarten sind, liegen nur wenige vor. Insbesondere bei hämatoonkologischen Patienten mit Fieber, als auch bei nicht-hämatoonkologischen Intensivpatienten mit schweren Infektionen existiert keine ausreichende Datenlage.

Die klinische Erfahrung zeigt jedoch, dass gerade bei diesen Patienten Therapieversagen auch bei nachgewiesener Empfindlichkeit der Keime gegen das eingesetzte Antibiotikum auftreten kann. Ursächlich müssen ausgeprägte Veränderungen der pharmakokinetischen Parameter durch pathophysiologische Einflussfaktoren im Rahmen der Infektion, welche die antibiotisch wirksame Fraktion des Medikamentes beeinflussen, in Betracht gezogen werden.

Die Vielzahl und Unterschiedlichkeit der bei schweren Infektionen und Sepsis auftretenden Einflussfaktoren können den für den Therapieerfolg entscheidenden Pharmakonspiegel in nicht vorhersehbarer Weise verändern. Die resultierende Aufhebung der Dosis-Wirkungsbeziehung macht eine körpergewichtsbezogene Dosierung unmöglich und stellt eine Indikation zur Bestimmung der Medikamentenspiegel dar.

In der vorliegenden Arbeit wurden ausgewählte mit Meropenem behandelte febrile hämatoonkologische Risikopatienten und internistische Patienten auf Intensivstation mit schweren und schwersten Infektionen untersucht.

Ziel der Untersuchung war:

(a) die Analyse der Pharmakokinetik von Meropenem bei febrilen hämatoonkologischen Patienten und bei nicht-hämatoonkologischen Intensivpatienten mit schweren Infektionen, 
(b) die Klärung der Frage, ob bei diesen kritisch kranken Patienten unter der üblichen Meropenem-Dosierung subtherapeutische Plasmakonzentrationen auftreten können, welche zu einem Therapieversagen führen,

(c) die Analyse verschiedener pathophysiologischer Veränderungen in Bezug auf die Infektion als Ursachen eines möglichen Therapieversagens während der antibiotischen Therapie mit Meropenem und

(d) das Aufzeigen von Möglichkeiten einer individuellen Dosierung in diesen Patientengruppen.

\subsection{Antibiotikatherapie kritisch kranker Patienten}

Bei der Antibiotikatherapie kritisch kranker Patienten müssen zum Erreichen eines guten klinischen Responses unter Vermeidung von Toxizität oder Resistenzentwicklung eine Vielzahl von klinischen, mikrobiologischen, pharmakologischen, epidemiologischen und pharmakodynamischen Faktoren beachtet werden:

- spezielles Erregerspektrum und Resistenzlage

- erreichbare Wirkkonzentration am Ort der Infektion

- die spezielle Pharmakodynamik (zeitabhängig/konzentrationsabhängig) des verwendeten Antibiotikums

- die Pharmakokinetik des verwendeten Antibiotikums in Abhängigkeit von den individuellen pathophysiologischen Konditionen des zu behandelnden Patienten.

Es sollte also das „richtige“ Antibiotikum am Ort der Infektionen in ausreichend hoher Konzentration über einen ausreichend langen Zeitraum wirken. Dabei sind die individuellen pathophysiologischen Gegebenheiten des zu behandelnden Patienten zu berücksichtigen.

Im Folgenden werden diese speziellen Aspekte unter besonderer Berücksichtigung der Antibiotikatherapie mit Meropenem erläutert. 


\subsubsection{Spezielles Erregerspektrum und Resistenzlage}

Die Auswahl eines Antibiotikums orientiert sich in erster Linie an der in-vitro-Sensibilität des nachgewiesenen Erregers. Bei Patienten mit schweren Infektionen kann jedoch häufig kein Keimnachweis abgewartet werden und in einigen Fällen ist kein Keimnachweis möglich. Die Antibiotikatherapie muss oft empirisch beginnen. Dabei sind das vermutete Erregerspektrum und die aktuelle Resistenzlage zu berücksichtigen. Auf Intensivstationen und auf hämatoonkologischen Stationen liegen vermehrt Antibiotikaresistenzen vor (Bodmann et al. 2001).

Meropenem hat ein breites antibakterielles Spektrum, welches die meisten grampositiven, gramnegativen inklusive Beta-Lactamase-Produzenten und Pseudomonaden sowie anaeroben Bakterien einschließt (Moellering, Jr. et al. 1989). Resistenzen gegen die meisten Keime sind eher ungewöhnlich. Es eignet sich nach allgemeiner Datenlage aufgrund seines breiten Spektrums in Bezug auf die in-vitro-Sensibilität zur empirischen Therapie von schweren nosokomialen Infektionen bei hämatoonkologischen Patienten (Cometta und Glauser 1996, Feld et al. 2000) und bei Intensivpatienten (Hurst und Lamb 2000, Schuster und Werdan 2000).

Bei hämatoonkologischen Patienten besteht oft eine erkrankungs- oder therapiebedingte Infektabwehrschwäche. Insbesondere zytostatische Therapie führt über Neutropenie zu einer Insuffizienz des Immunsystems. Fieber und Infektion sind die häufigsten Komplikationen während einer Neutropenie und ein bedeutsames klinisches Problem (Klastersky 1998). Patienten mit Leukämien und Lymphomen, die hinsichtlich ihrer Grunderkrankung eine kurative Heilungschance haben, versterben in signifikanter Zahl an schweren Infektionen infolge Chemotherapie-bedingter Neutropenie.

Bei febrilen hämatoonkologischen Patienten in Neutropenie gelingt häufig kein Keimnachweis. $40 \%$ dieser Patienten haben weder eine mikrobiologisch, noch eine klinisch definierte Infektion (Klastersky 1998). Fieber ist häufig das einzige Zeichen einer Infektion. Letzteres wird als "Fever of unknown origin" (FUO) bezeichnet. Bei den mikrobiologisch gesicherten Infektionen kam es im Laufe der letzten Jahrzehnte in dieser Patientengruppe zu einem Wandel des Erregerspektrums von gramnegativen Keimen zu grampositiven Kokken. Infektionen mit grampositiven Kokken sind häufig durch koagulase-negative Staphylokokken oder Corynebakterium jeikeum bedingt, bei denen die klinische Situation im Gegensatz zu Infektionen durch gramnegative Erreger weniger fulminant verläuft. Staphylococcus aureus, Viridans-Streptokokken und Pneumokokken können hingegen rasch progrediente Infektionen hervorrufen, die ohne adäquate Therapie infaust verlaufen können (Link et al. 2003). Grampositive Keime repräsentieren $70 \%$ der mikrobiologisch nachgewiesenen Infektionen bei 
neutropenischen Patienten. In verschiedenen Studien wurde die Effektivität von Meropenem bei der Behandlung von Infektionen neutropenischer Patienten demonstriert. Es ist geeignet zur empirischen Mono- oder Kombinationstherapie febriler Episoden neutropenischer Hochrisikopatienten (Behre et al. 1998, Cometta et al. 1996, de la Camara R. et al. 1997).

Infektionen auf Intensivstationen haben ihrerseits spezifische Merkmale. Das Risiko einer Infektion steigt mit der Dauer des Aufenthalts und der Anzahl der invasiven Eingriffe (Jarvis et al. 1991, Richards et al. 1999, Vincent et al. 1995).

Die häufigsten Infektionen, die auf der Intensivstation erworben werden, sind Pneumonien, Harnwegsinfektionen und Blutstrom-Infektionen (Richards et al. 1999). Weitere häufige Infektionen sind gastrointestinale Infekte, nicht-pneumonische Infektionen der unteren Atemwege, Wundinfekte sowie Infektionen der Haut und Weichteile. Richards et al. zeigten, dass $87 \%$ der Patienten mit Blutstrominfektionen einen zentralen Zugang hatten, 86 \% der Patienten mit nosokomialen Pneumonien künstlich beatmet wurden und 95 \% der Harnwegsinfektionen mit Urinkathetern assoziiert waren (Richards et al. 1999). Ein signifikant erhöhtes Infektionsrisiko wurde bei Patienten mit Polytrauma, Verbrennungen, großen operativen Eingriffen und Stressulkusprophylaxe beobachtet (Vincent et al. 1995).

Die häufigsten Infektionserreger sind Enterobacteriaceae (34,4 \%), Staphylococcus aureus $(30,1 \%)$, Pseudomonas aeruginosa $(28,7 \%)$, koagulase-negative Staphylokokken $(17,1 \%)$ und Pilze (17,1\%), wobei auch Mehrfachinfektionen berücksichtigt wurden (Vincent et al. 1995). Eine kalkulierte Antibiotikatherapie muss so ausgewählt werden, dass gegen die beim jeweiligen Krankheitsbild häufig auftretenden Erreger eine sichere bakterizide Wirkung erwartet werden kann. Carbapeneme sind geeignet zur Therapie nosokomialer Infektionen auf Intensivstation. Sie werden sowohl als Monotherapeutika als auch bei schweren Infektionen und Sepsis in Kombination mit anderen Antibiotika eingesetzt (Hurst und Lamb 2000, Schuster und Werdan 2000).

\subsubsection{Wirkkonzentrationen von Antibiotika am Ort der Infektion}

Neben der in-vitro-Sensibilität des zu bekämpfenden Erregers sind ausreichend hohe Wirkkonzentrationen des Antibiotikums am Ort der Infektion essentielle Voraussetzung für eine effektive Keimelimination unter Vermeidung von Resistenzentwicklung. Ein Antibiotikum sollte also so hoch dosiert werden, dass es am Infektionsherd eine therapeutisch effiziente Konzentration erreicht (Sauermann et al. 2005). Sollte das verwendete Antibiotikum das infizierte Gewebe nicht erreichen oder können keine ausreichenden Konzentrationen aufrecht- 
erhalten werden, so kann es trotz in-vitro-Empfindlichkeit der Keime zu einem Therapieversagen kommen (Tomaselli et al. 2004).

Nach der intravenösen Gabe eines hydrophilen Antibiotikums wie Meropenem folgt die schnelle initiale Verteilung des Pharmakons im peripheren Gefäßsystem (zentrales Kompartiment). Von dort kommt es dem Konzentrationsgradienten folgend zum Übertritt des Pharmakons über die Kapillarwand in den interstitiellen Raum der Gewebe (peripheres Kompartiment). Das Interstitium stellt häufig den Wirkort der meist extrazellulär vorliegenden Erreger dar. Das Penetrationsverhalten von Antibiotika ins interstitielle Gewebe ist von der Molekülgröße, der Lipophilie, dem Ausmaß der Proteinbindung, dem pH-Wert von Körperflüssigkeiten, der Beschaffenheit der Kapillarwände, der Organdurchblutung und dem Vorhandensein von aktiven Transportmechanismen an physiologischen Barrieren abhängig (Sauermann et al. 2005). Diese physiologischen Einflussgrößen können unter pathologischen Konditionen oft unvorhersehbar verändert sein und das Plasma-Gewebs-Äquilibrium stark verzögern. So kann ein verminderter Proteingehalt im Rahmen einer Sepsis, ein verminderter pH-Wert bei Infektion oder eine reduzierte Durchblutung im entzündeten Gewebe den wirksamen lokalen Medikamentenspiegel stark verändern. Die pharmakokinetischen Daten aus gesundem Gewebe können also nicht unreflektiert auf entzündetes Gewebe übertragen werden.

Meropenem wird in der Regel in Standarddosierung als intravenöse Kurzinfusion verabreicht. Aufgrund der überwiegend renalen Elimination sollte bei Nierenfunktionseinschränkung eine Dosisreduktion oder Verlängerung des Dosierungsintervalls erfolgen. Meropenem hat ein niedriges Molekulargewicht von 383,5 Da. Es existiert keine wesentliche Proteinbindung ( $2 \%$ ), so dass es überwiegend in freier Form vorliegt (Robatel et al. 2003). Die Pharmakokinetik von Meropenem folgt einem 2-Kompartment-Modell. In der initialen Phase nach Infusion fällt die Konzentration im zentralen Kompartiment aufgrund des hohen Konzentrationsgradienten zum peripheren Kompartiment sehr stark ab (Verteilungsphase). Die Verteilung in das periphere Kompartiment vollzieht sich bis ein Steady-state erreicht ist, d.h. bis die freien Konzentrationen im zentralen und im peripheren Kompartiment etwa gleich groß sind. Nach Erreichen des Steady-state wird die Konzentration im zentralen Kompartiment durch die Elimination über die Niere bestimmt. Durch den weiteren Abfall der Konzentration im zentralen Kompartiment kommt es nach Erreichen des Steady-state zu einer Umkehr des Konzentrationsgradienten, d.h. es kommt zu einer Rückverteilung des Pharmakons aus dem peripheren in das zentrale Kompartiment, wo es schließlich eliminiert wird. Ein Teil des 
Medikamentes wird wahrscheinlich durch unspezifische Hydrolasen in seinen inaktiven Metaboliten abgebaut.

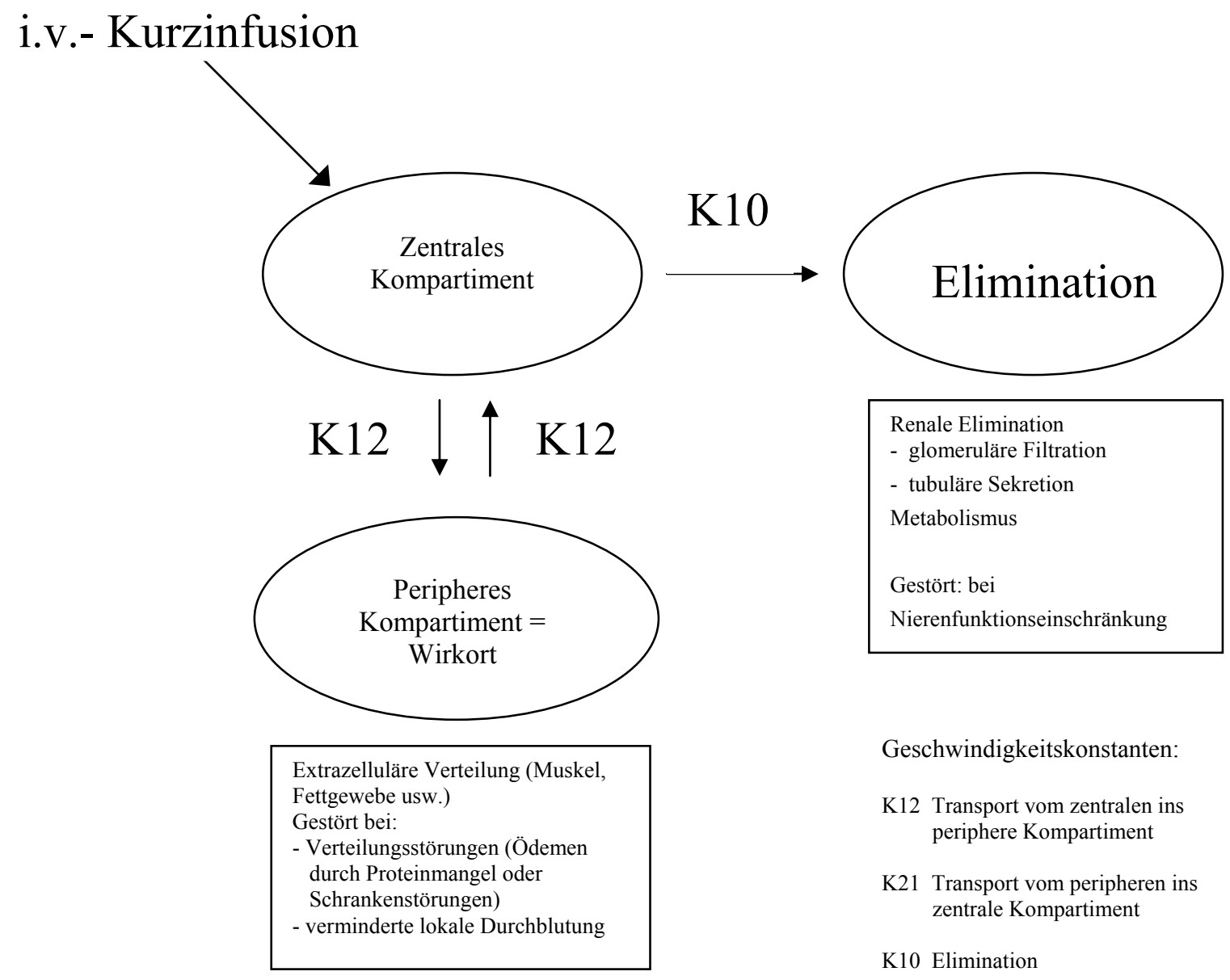

Abbildung1: 2-Kompartment-Modell zur Beschreibung der Pharmakokinetik von Meropenem

Unter physiologischen Bedingungen besitzt Meropenem eine gute Gewebspenetration (Hutchison et al. 1995). Nach der Gabe von 1000 mg Meropenem wurden nach Literaturangaben im Respirationstrakt Spiegel zwischen 0,54 bis 6,39 mg/l (Byl et al. 1999), in intraabdominellen Geweben von 2,01 bis $14,6 \mathrm{mg} / \mathrm{l}$ und in der Haut von 5,3 bis 8,76 mg/l gemessen. Im Liquor werden aufgrund der physiologischen Barriere (Blut-Hirn-Schranke) niedrige Konzentrationen erreicht, allerdings sind die Konzentrationen bei entzündlichen Meningen deutlich erhöht $(1 \mathrm{mg} / \mathrm{l})$. Auch in anderen Geweben mit entzündlichen Veränderungen konnten ausreichend hohe Medikamentenkonzentrationen gefunden werden. Neue Verfahren der Mikrodialyse zur direkten Messung der interstitiellen Medikamentenkonzentration haben gezeigt, dass die Meropenem-AUC in der Peritonealflüssigkeit bei Patienten mit schwerer Peritonitis und septischem Schock $73 \pm 15 \%$ der Plasma-AUC erreichten. In der üblichen Dosierung von 3-mal $1000 \mathrm{mg} / \mathrm{d}$ wurden in dieser Patientengruppe ausreichende 
Spiegel zur Bekämpfung sensibler, aber nicht immer für intermediäre Keime beobachtet (Karjagin et al. 2008). Das Penetrationsverhalten von Meropenem in die Lunge bei Patienten mit Pneumonie und Pleuraempyem wurde ebenfalls mit dem Mikrodialyseverfahren untersucht. Es zeigte sich auch hier eine gute Gewebspenetration. Im Lungeninterstitium wurden 40-60 \% der Serum-AUC erreicht (Tomaselli et al. 2004).

Meropenem besitzt somit aufgrund seiner chemischen Eigenschaften ein gutes Penetrationsverhalten in entzündliches Gewebe. Nach Erreichen des Steady-state entspricht die Wirkkonzentration im Interstitium nahezu der Medikamentenkonzentration im Plasma. Plasmakonzentrationen sind somit als Surrogatparameter für Wirkspiegel im entzündlichen Gewebe geeignet (Cars 1981, Tomaselli et al. 2004) und wurden auch in der vorliegenden Studie verwendet.

\subsubsection{Pharmakodynamik}

Als weiterer Einflussfaktor zur effektiven antimikrobiellen Therapie ist die Pharmakodynamik des verwendeten Antibiotikums zu berücksichtigen. Bezüglich der verschiedenen Arten der antibiotischen Wirksamkeit werden 2 Hauptgruppen unterschieden:

- Antibiotika mit konzentrationsabhängiger Wirkung

- Antibiotika mit zeitabhängiger Wirkung.

Bei Antibiotika mit konzentrationsabhängiger Wirkung sind eine ausreichend hohe Spitzenkonzentration $\left(\mathrm{C}_{\max } / \mathrm{MIC}\right)$ oder Fläche unter der Zeit-Konzentrations-Kurve (AUC/MIC) am Ort der Infektion Voraussetzung für die maximale Wirksamkeit (Aminoglykoside und Fluorchinolone) (Craig 1998, Forrest et al. 1993, Freeman et al. 1997). Das heißt höhere Konzentrationen führen zu einer rascheren und intensiveren bakteriziden Wirkung.

Bei den zeitabhängigen Antibiotika sind ausreichend lange Zeiträume, in denen die Konzentration des Therapeutikums die minimale Hemmkonzentration (MIC) des zu bekämpfenden Keims überschreitet ( $\% \mathrm{~T}>\mathrm{MIC})$, Voraussetzung für einen ausreichenden antibiotischen Effekt (Beta-Lactam-Antibiotika, Carbapeneme) (Turnidge 1998).

Meropenem gehört zur Gruppe der Antibiotika mit zeitabhängiger Wirkung. Zur wirksamen Therapie müssen also ausreichend hohe Konzentrationen über einen ausreichend langen Zeitraum aufrechterhalten werden.

Verschiedene Autoren zeigten im Tiermodell eine maximale bakterizide Aktivität, wenn die minimale Hemmkonzentration (MIC) des zu bekämpfenden Keims über mindestens $40 \%$ des 
Dosierungsintervalls aufrechterhalten wird (Mattoes et al. 2004, Ong et al. 2007). Es sollte allerdings berücksichtigt werden, dass es bei Unterschreitung der MIC zu einem Nachwachsen der Keime (Regrowth) oder zur Resistenzentwickung kommen kann (Hyatt et al. 1995, Mouton und Vinks 2005). Die Wahrscheinlichkeit einer mikrobiologischen Erradikation erhöht sich in vivo bei deutlich längerer Überschreitung der Hemmkonzentrationen. So wurde bei febrilen neutropenischen Patienten mit Bakteriämie zum Erreichen eines 80 \% klinischen Responses eine Überschreitung der minimalen Hemmkonzentration (\% $\%$ MIC) von über $76 \%$ des Dosierungsintervalls gefunden (Ariano et al. 2005).

Für eine effektive antibakterielle Wirksamkeit ist somit bei kritisch kranken Patienten die Überschreitung der Hemmkonzentration des zu bekämpfenden Erregers über mindestens 60$70 \%$ des Dosierungsintervalls erforderlich (Cars 1997, Craig 1998, Turnidge 1998). Bei immuninkompetenten Patienten wird sogar eine Überschreitung der minimalen Hemmkonzentration des zu bekämpfenden Keims während des gesamten Dosierungsintervalls empfohlen (Lamoth et al. 2009, Pea und Viale 2006). Die Effektivität von Beta-Lactam-Antibiotika erhöht sich deutlich bei Konzentrationen des 4- bis 5-fachen der MIC des zu bekämpfenden Keims (Bowker et al. 1996, Mouton und Vinks 1996). Ein postulierter postantibiotischer Effekt (PAE) von Meropenem wird kontrovers diskutiert. Carbapeneme unterscheiden sich von anderen Beta-Lactamen indem sie einen postantibiotischen Effekt sowohl gegen grampositive als auch gegen gram-negative Bakterien aufweisen. Die meisten Studien sind jedoch in vitro, nur wenige Studien erfolgten in vivo. Die meisten ermittelten PAE sind widersprüchlich und in hohem Maß von der verwendeten Methode abhängig (Hanberger et al. 1995). Der PAE für Pseudomonas aeruginosa liegt bei 1-4 Stunden. Die klinische Relevanz des postantibiotischen Effekts ist bisher nicht sicher belegt (Fuentes et al. 1995) und kann im Sinne der Therapiesicherheit nicht einkalkuliert werden.

\subsubsection{Pharmakokinetik in Abhängigkeit von pathophysiologischen Einflüssen}

Die Serumkonzentrationen von Medikamenten sind eine Resultante verschiedener Einflussfaktoren (Pea und Viale 2006). Pathophysiologische Veränderungen oder iatrogene Interventionen bei kritisch kranken Patienten haben einen großen Einfluss auf die Elimination und Verteilung insbesondere von hydrophilen Antibiotika. Aufgrund der großen intra- und interindividuellen pharmakokinetischen Variablilität kommt es häufiger als gemeinhin angenommen zu Über- und Unterdosierungen und somit zur Störung der Zielkonzentration.

Unterdosierungen können aufgrund erhöhter Verteilungsvolumina oder erhöhter Medikamentenclearances auftreten. Das Verteilungsvolumen insbesondere hydrophiler Medikamente mit 
überwiegend extrazellulärer Verteilung ist erhöht bei Extravasation mit Ödemen infolge von Proteinmangel oder Störungen der Gefäßpermeablilität, wie sie im Rahmen einer Sepsis auftreten kann, weiterhin bei Aszites und Ergüssen oder durch intensivierte Infusionstherapie. Das effektiv zirkulierende Volumen ist aufgrund der Verteilungsstörungen bei Sepsis mit erhöhter Gefäßpermeabilität oft erniedrigt. Die Notwendigkeit der Flüssigkeitsgabe zum Auffüllen des zentralen Kompartiments führt zur weiteren Extravasation mit Ödemen, was wiederum zu einer Vergrößerung des Verteilungsvolumens führt. Eine erhöhte Clearance kann z.B. bei hyperdynamischen Zuständen während einer Sepsis (Pinder et al. 2002), bei forcierter Diurese oder dem Gebrauch hämodynamisch aktiver Medikamente (Pea und Furlanut 2001) auftreten. Überdosierungen treten gehäuft infolge von akutem Organversagen wie Niereninsuffizienz auf, z.B. als Komplikation einer Sepsis mit verminderter Ausscheidung.

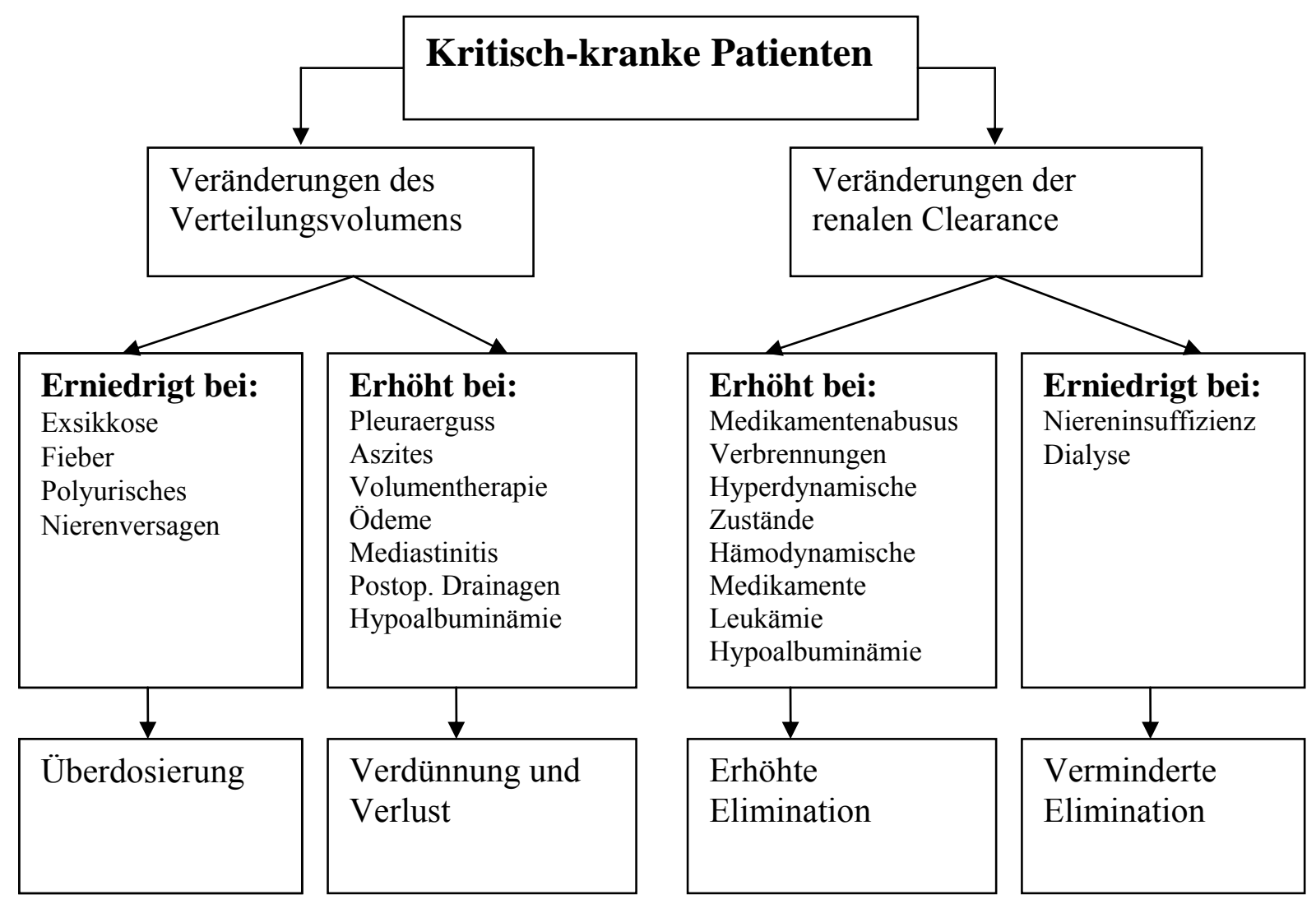

Abbildung 2: Pathophysiologische oder iatrogene Konditionen mit Beeinflussung der Verteilung und Elimination von Antibiotika modifiziert nach Pea und Viale (2006), S.1769

All diese Konditionen können innerhalb eines Patienten koexistieren. Der Zustand eines Patienten mit Sepsis oder septischem Schock kann sich aus dem Krankheitsgeschehen heraus oder infolge therapeutischer Maßnahmen innerhalb weniger Stunden erheblich ändern und eine veränderte Pharmakokinetik nach sich ziehen. Vorhersagen über den Medikamenten- 
spiegelverlauf sind nicht oder nur eingeschränkt möglich. Allein die direkte Messung erlaubt eine Beurteilung der tatsächlich vorliegenden Serumkonzentration. Die antimikrobielle Therapie bei kritisch kranken Patienten profitiert somit häufig vom therapeutischen Drug monitoring (Pea et al. 2005a).

\subsection{Die Eigenschaften von Meropenem}

Das Breitspektrumantibiotikum Meropenem gehört zur Gruppe der parenteralen Carbapeneme. Es ist weitgehend stabil gegenüber der renalen Dehydropeptidase 1, so dass die Kombination mit dem Dehydropeptidase-1-Inhibitor Cilastatin wie bei Imipenem entfällt (Fish und Singletary 1997, Moon et al. 1997). Sein breites antibakterielles Spektrum schließt die meisten grampositiven, gramnegativen und anaeroben Bakterien ein (Moellering, Jr. et al. 1989). Resistenzen gegen Meropenem sind für die meisten Keime eher ungewöhnlich (Hurst und Lamb 2000).

\subsubsection{Antibakterielles Spektrum}

Meropenem zeigt im Vergleich zu Imipenem im gramnegativen Bereich eine höhere Wirksamkeit. Auch im grampositiven Bereich zeigt es eine deutliche antibakterielle Wirkung (neben Staphylococcus aureus auch andere Staphylokokken), allerdings geringer als Imipenem.

Gegen sporenlose Anaerobier (z.B. Bacteroides fragilis) und die meisten Clostridien-Arten (z.B. Clostridium perfringens) ist die Wirkung gleich. Oxacillin-resistente Staphylokokken, Enterococcus faecium, Stenotrophomonas maltophilia, Xanthomonas maltophilia, Mycoplasmen, Chlamydien, Legionellen und die meisten Mykobakterien-Arten sind resistent.

In Kombination mit anderen Antibiotika, z.B. mit einem Aminoglykosid gegen Pseudomonas aeruginosa oder mit Vancomycin gegen Staphylokokken, wirkt Meropenem synergistisch.

Gramnegative Aerobier:

Enterobacteriaceae sind hoch sensibel gegenüber Meropenem. Die minimal erforderliche Konzentration zur Hemmung von 90 \% der klinischen Isolate (MIC90) liegt bei 0,06 bis 1 $\mathrm{mg} / \mathrm{l}$. Pfaller und Jones konnten zeigen, dass Meropenem gegen Enterobacteriaceae eine höhere Aktivität entwickelt, als alle anderen getesteten Antibiotika (Imipenem, Ciprofloxacin, Piperacillin/Tazobactam, Cefotaxim, Ceftazidim) (Pfaller und Jones 1997). Andere gramnegative Aerobier einschließlich Haemophilus influenzae und Neisseria meningitidis sind ebenfalls gegen Meropenem sensibel. Für Pseudomonas aeruginosa liegt die MIC90 zwischen 
4-8 mg/l. Dieser Keim ist als intermediär (2-8 mg/l) einzustufen. Insbesondere auf Intensivstationen und hämatoonkologischen Stationen liegt die Sensibilität im oberen intermediären Bereich (8 mg/l) (Goossens 2000).

Grampositive Aerobier:

Gegen grampositive Aerobier zeigt Meropenem eine geringere Aktivität als Imipenem. Der Methicillin-sensitive Staphylococcus aureus sowie Streptococcus pneumoniae und pyogenes sind hoch sensibel gegenüber Meropenem (MIC90 = 0,06-1,0 mg/l). Die minimale Hemmkonzentration, um $90 \%$ der klinischen Isolate von Methicillin-sensitivem Staph.epidermidis zu hemmen liegt bei $4 \mathrm{mg} / \mathrm{l}$. Die Aktivität von Meropenem gegenüber Enterococcus faecalis ist variabel (MIC90 = 8-32 $\mathrm{mg} / \mathrm{l})$, aber letztlich gering. Meropenem zeigt keine Aktivität gegenüber Methicillin-resistenten Staphylokokken (MRS) und Enterococcus faecium (MIC90 $>16 \mathrm{mg} / \mathrm{l})$. Insbesondere auf Intensivstationen ist mit einer erhöhten Resistenzrate zu rechnen, so dass alle Enterokokken und MRS als resistent gegenüber Meropenem einzustufen sind.

Anaerobier:

Sowohl nach Pfaller und Jones (Pfaller und Jones 1997), die 2257 Isolate von anaeroben Bakterien testeten, als auch nach Edwards (Edwards 1995) ist Meropenem gegen die meisten Anaerobier wirksam. Clostridium difficile, Clostridium perfringens und Bacteroides fragilis sind hochsensibel (MIC90 = 0,06-2,0 mg/l) (Turner 2000).

\subsubsection{Pharmakokinetik von Meropenem}

Die meisten Studien zur Pharmakokinetik von Meropenem beschäftigen sich mit gesunden Erwachsenen oder Patienten mit leichten Infektionen (Kelly et al. 1995, Wise et al. 1990). Wenige Studien haben sich darüber hinaus mit der Pharmakokinetik von Meropenem bei Patienten mit schweren Infektionen, Niereninsuffizienz, Hämofiltration, Leberinsuffizienz oder febriler Neutropenie auseinandergesetzt. Die Datenlage bei diesen Patientengruppen ist unzureichend und lässt nur wenige Rückschlüsse auf den Einfluss erkrankungsbedingter Veränderungen auf die Pharmakokinetik des untersuchten Antibiotikums zu. Entsprechend vielfältig sind die Vorschläge zur Dosismodifikation bei speziellen Patientengruppen.

\section{Meropenem bei gesunden Erwachsenen}

Die Pharmakokinetik von Meropenem bei gesunden Erwachsenen ist durch folgende Eigenschaften gekennzeichnet: Die Spitzenkonzentration $\left(\mathrm{C}_{\max }\right)$ und die Fläche unter der ZeitKonzentrations-Kurve $\left(\mathrm{AUC}_{0-\infty}\right)$ zeigen eine lineare Beziehung zur applizierten Dosis $(\mathrm{Bax}$ et 
al. 1989). Nach 30minütiger Infusion von 500 bzw. $1000 \mathrm{mg}$ Meropenem bei gesunden Freiwilligen liegen die Spitzenkonzentrationen im Plasma bei 22-27 bzw. 50-60 mg/l. Die $\mathrm{AUC}_{0-\infty}$ liegt bei der Dosis von $500 \mathrm{mg}$ bei $28-40 \mathrm{mg} /(1 \cdot \mathrm{h})$ und bei der Dosis von $1000 \mathrm{mg}$ bei $66-77 \mathrm{mg} /(1 \cdot \mathrm{h})$.

Meropenem hat im Vergleich zu anderen Beta-Lactam-Antibiotika ein relativ großes Verteilungsvolumen von ca. 12,5-25 1 ( 0,17-0,35 1/kg ). Die Verteilung findet überwiegend extrazellulär statt.

Die Elimination erfolgt überwiegend renal, vor allem durch glomeruläre Filtration, zu einem geringeren Anteil auch durch tubuläre Sekretion, mit einer Plasma-Halbwertszeit von 1 bis 1,4 Stunden (Bax et al. 1989, Christensson et al. 1992). Die renale Ausscheidung von Meropenem bei Patienten mit normaler Nierenfunktion liegt bei 70-80 \% der applizierten Dosis (Bax et al. 1989, Dreetz et al. 1996). 54-79 \% werden unverändert im Urin ausgeschieden. Die renale Meropenem-Clearance beträgt 143-200 ml/min und übersteigt die glomeruläre Filtrationsrate $(\sim 120 \mathrm{ml} / \mathrm{min})$ um 40-60 \%. Diese Überschreitung ist durch tubuläre Sekretion und unspezifische Hydrolyse zu erklären.

Bei der gleichzeitigen Gabe des Urikosurikums Probenecid wird die tubuläre Sekretion kompetitiv gehemmt. Die renale Meropenem-Clearance fällt auf $129 \mathrm{ml} / \mathrm{min}$ und liegt dann in der Größenordnung der glomerulären Filtrationsrate (Bax et al. 1989, Nilsson-Ehle et al. 1991). Die Rate der Ausscheidung von Meropenem im Urin ist unabhängig von der Dosis (Bax et al. 1989).

Die nicht-renale Clearance wird überwiegend durch den Arzneimittelmetabolismus repräsentiert. Die Umwandlung von Meropenem in seinen unwirksamen Metaboliten (ICI 213689) erfolgt durch unspezifische Hydrolyse im Plasma, Umwandlung durch die renale Dehydropeptidase 1 und möglicherweise durch bestimmte Enzymsysteme des Gewebes. 2 \% der applizierten Meropenem-Dosis werden in den Faeces, der Rest als inaktiver Metabolit (ICI 213689) nach Öffnung des Beta-Lactamrings im Urin ausgeschieden.

Die Plasmaproteinbindung von Meropenem ist gering (2\%) (Simon und Stille 1997).

Bei Patienten mit Niereninsuffizienz sinkt die renale Clearance von Meropenem proportional zur Kreatinin-Clearance. Es wird entsprechend eine Dosisreduktion oder eine Verlängerung des Dosierungsintervalls empfohlen (Chimata et al. 1993, Christensson et al. 1992).

Erwartungsgemäß zeigt eine veränderte Leberfunktion keinen Einfluss auf die Elimination (Christensson et al. 1992, Leroy et al. 1992, Wise et al. 1990) 


\section{Meropenem bei Patienten mit schweren Infektionen}

Schon in früheren Studien wurde eine Veränderung der Pharmakokinetik von Beta-LactamAntibiotika bei Patienten mit febrilen Infektionen beobachtet. Für Ceftazidim wurden unter der Infektion ein erhöhtes Verteilungsvolumen sowie eine erhöhte renale und totale Clearance nachgewiesen (Ljungberg und Nilsson-Ehle 1988).

Nur wenige Studien befassen sich mit der Pharmakokinetik von Meropenem bei schweren Infektionen. Bedikian et al. beschäftigten sich mit der Pharmakokinetik von Meropenem bei postoperativen Patienten mit intraabdominellen Infektionen. Diese Patienten hatten ein erhöhtes Verteilungsvolumen $\left(\mathrm{V}_{\mathrm{d}}=0,38 \mathrm{1} / \mathrm{kg}\right)$ und eine erhöhte totale Meropenem-Clearance $(\mathrm{Cl}=315 \pm 71 \mathrm{ml} / \mathrm{min})$. Die Spitzenkonzentrationen und die AUC waren nach Applikation von 1000 mg Meropenem um je ca. 20-30 \% im Vergleich zu gesunden Probanden auf 47,6 $17,58 \mathrm{mg} / \mathrm{l}$ und 57,5 $\pm 20,12(\mathrm{mg} \cdot \mathrm{h}) / 1$ vermindert. Die renale Clearance $(138 \pm 89,2 \mathrm{ml} / \mathrm{min})$ und die Eliminationskonstante $(0,68 \pm 0,12)$ blieben unverändert.

Obwohl fast alle Patienten eine normale Nierenfunktion hatten, konnte keine Korrelation zwischen der Meropenem-Gesamtclearance und der Kreatinin-Clearance festgestellt werden. Trotz der pharmakokinetischen Veränderungen wurde eine Dosis von 1000 mg alle 8 Stunden als ausreichend erachtet (Bedikian et al. 1994).

\section{Meropenem bei neutropenischen Patienten mit Fieber}

Nyhlen et al. untersuchten die Pharmakokinetik von Meropenem bei febrilen neutropenischen Patienten. Diese Daten wurden mit den pharmakokinetischen Parametern aus einer früheren Studie mit gesunden jüngeren (20-34 Jahre) und älteren (67-80 Jahre) Freiwilligen verglichen. Die Verteilungsvolumina von Meropenem waren in der Gruppe der febrilen Neutropeniker

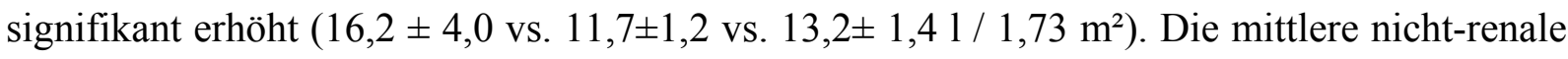
Clearance war ähnlich wie in der Gruppe der jungen gesunden Freiwilligen, aber signifikant größer als bei den älteren gesunden Freiwilligen $(75 \pm 28,1$ vs. $66 \pm 17,5$ vs. $39 \pm 14,5 \mathrm{ml} / \mathrm{min}$ 1,73 $\mathrm{m}^{2}$ ), obwohl das Durchschnittsalter der Neutropeniker bei 61 (36-82) Jahren lag. Die totale und renale Clearance $\left(209 \pm 92,4\right.$ und $\left.150 \pm 70,1 \mathrm{ml} / \mathrm{min} \cdot 1,73 \mathrm{~m}^{2}\right)$, wie auch die tubuläre Sekretion $\left(49 \pm 41 \mathrm{ml} / \mathrm{min} \cdot 1,73 \mathrm{~m}^{2}\right)$ von Meropenem tendierten $\mathrm{zu}$ höheren Werten als bei gesunden Freiwilligen. Aufgrund der großen Variabilität dieser Daten ergab sich jedoch keine statistische Signifikanz.

Die kinetische Studie wurde wiederholt, nachdem die Fieberphase überwunden und die neutrophilen Granulozyten angestiegen waren. Die pharmakokinetischen Parameter änderten 
sich nur geringfügig. Allerdings wurde ein Rückgang der glomerulären Filtrationsrate und der renalen Clearance $\left(141 \pm 78 \mathrm{ml} / \mathrm{min} \cdot 1,73 \mathrm{~m}^{2}\right)$ beobachtet.

Fieber und / oder Infektion scheinen die renale Elimination von Meropenem zu erhöhen. Diese Hypothese wird durch den Rückgang der glomerulären Filtrationsrate während der Therapie unterstützt.

Möglicherweise sind das erhöhte Verteilungsvolumen und der Anstieg der non-renalen Clearance auf die Neutropenie, die zytostatische Therapie und / oder auf die akute Infektion zurückzuführen (Nyhlen et al. 1997).

\section{Meropenem bei Patienten mit Nierenfunktionseinschränkungen}

Da Meropenem überwiegend über die Niere ausgeschieden wird, führt eine renale Funktionseinschränkung zu einer verminderten Elimination dieser Substanz. Es kommt zu einer verminderten totalen Clearance, einer verminderten renalen Clearance und einer verlängerten Eliminationshalbwertszeit von Meropenem (Wiseman et al. 1995). Verschiedene Autoren berichten von einer Korrelation zwischen Meropenem- und Kreatinin-Clearance (Bax et al. 1989, Chimata et al. 1993, Christensson et al. 1992, Leroy et al. 1992). Bei Patienten mit chronischer Niereninsuffizienz wurde ebenfalls ein linearer Zusammenhang zwischen Meropenem- und Kreatinin-Clearance gezeigt.

Bei fortschreitendem Abfall der Nierenfunktion kommt es allerdings zu einem Anstieg der nicht-renalen Clearance von Meropenem (Chimata et al. 1993). Bei Patienten mit normaler Nierenfunktion lag die nicht-renale Meropenem-Clearance bei maximal $20 \%$. Bei Patienten mit Niereninsuffizienz und Abfall der glomerulären Filtrationsrate auf 5-29 ml/min stieg sie bis auf $50 \%$ an. Der Haupteliminationsweg der nicht-renalen Clearance ist die Metabolisierung der Substanz (Christensson et al. 1992).

Für Imipenem hat sich gezeigt, dass die Pharmakokinetik bei Patienten mit Niereninsuffizienz als Akutkomplikation anders beeinflusst wird als bei Patienten mit chronischer terminaler Niereninsuffizienz. Die nicht-renale Clearance von Imipenem bei Patienten mit akutem Nierenversagen ist etwa doppelt so groß wie bei Patienten mit terminaler Niereninsuffizienz (Mueller et al. 1993). Dieser Zusammenhang wurde nicht bei Meropenem beobachtet. Hier wurde zwischen beiden Gruppen eine vergleichbare nicht-renale Clearance festgestellt. Der Grund für diese Unterschiede ist unklar. Als mögliche Erklärung wird die höhere Stabilität von Meropenem im Gegensatz zu Imipenem gegenüber unspezifischer Hydrolyse im Plasma gesehen (Tegeder et al. 1999). 
Einfluss von kontinuierlicher veno-venöser Hämofiltration (CVVHF): Verschiedene Autoren haben sich mit der Pharmakokinetik von Meropenem bei kontinuierlicher veno-venöser Hämofiltration (CVVHF) beschäftigt (Giles et al. 2000, Tegeder et al. 1999, Thalhammer et al.1998, Valtonen et al. 2000, Ververs et al. 2000).

Die kontinuierliche veno-venöse Hämofiltration ist die bevorzugte Nierenersatztherapie bei Intensivpatienten mit akutem Nierenversagen und Multiorganversagen. Sie ermöglicht unabhängig von der Kreislaufsituation des Patienten einen konstanten Fluss und eine hohe Filtrationsrate von etwa 20-30 1/d (Dehne und Kroh 1995, Mueller et al. 1993, Vos et al. 1992). Die dadurch erreichte Kreatinin-Clearance liegt bei $25-50 \mathrm{ml} / \mathrm{min}$ (Thalhammer et al. 1998).

Die Elimination verschiedener Medikamente ist abhängig von den Charakteristika der verwendeten Membran (Porengröße, Filteroberfläche, Absorption, Filtermaterial, Elektrostatik) und den Charakteristika des Medikamentes (Molekulargewicht, Proteinbindung, Wasserlöslichkeit, Ladung) (Thalhammer et al. 1998).

Der Fluss von Meropenem über die verschiedenen zur Verfügung stehenden Filtermembranen ist exzellent. Die Siebkoeffizienten liegen bei $\sim 1$ (Giles et al. 2000). Meropenem ist also frei filtrierbar. Der Fluss über die Filtermembran entspricht somit der ungebundenen Medikamentenfraktion von ca. 98 \%. Die Filterclearance von Meropenem entspricht also fast exakt der Filtrationsrate (Giles et al. 2000).

Die Clearance von Meropenem während der Hämofiltration bei akutem Nierenversagen steigt somit um die Meropenem-Clearance durch den Filter. Sie ist vergleichbar mit einer renalen Clearance bei Nierenrestfunktion von 10-25 ml/min. Nach Giles et al. korreliert die totale Meropenem-Clearance allerdings nicht mit der Filtrationsrate. Grund dafür ist die große Variabilität der nicht-renalen Clearance (15-70 ml/min). Die Halbwertszeit liegt bei ca. 5 (2,37,8) Stunden. Das Verteilungsvolumen ist auf 0,35 1/kg erhöht (normal 0,25 1/kg). Die Ursache liegt wahrscheinlich in der Erhöhung des extrazellulären Wassers.

Die Pharmakokinetik von Meropenem bei Patienten mit Nierenversagen wird also entscheidend durch CVVHF verändert. Etwa 50 \% der Substanz werden hierdurch extrakorporal eliminiert. Eine Dosisanpassung ist erforderlich. Die empfohlenen Dosen liegen bei $1000 \mathrm{mg}$ alle 8-12 h (Giles et al. 2000).

Einfluss von Dialyse: Meropenem wird effizient durch intermittierende Hämodialyse eliminiert, so dass zusätzliche Meropenem-Dosen nach jeder Hämodialysesitzung infundiert werden sollten. Nach Chimata et al. sinkt die Eliminationshalbwertszeit während der 
Hämodialyse von 7 auf 2,9 Stunden. Eine zusätzliche Gabe von 500 mg Meropenem am Ende der Dialyse wird empfohlen (Chimata et al. 1993).

\section{Meropenem und Leberfunktion}

Leberfunktionsstörungen scheinen keinen Einfluss auf die Pharmakokinetik von Meropenem $\mathrm{zu}$ haben. Thyrum et al. fanden keine signifikanten Unterschiede der pharmakokinetischen Parameter $\left(\mathrm{C}_{\max }, \mathrm{AUC}\right.$, Clearance, Verteilungsvolumen und Akkumulationsrate) bei Patienten mit chronisch stabiler alkoholischer Leberzirrhose im Vergleich zu einem gesunden Patientenkollektiv. Eine Dosisanpassung aufgrund einer Leberfunktionsstörung scheint nicht erforderlich (Thyrum et al. 1997).

Auch Giles et al. sahen keinen Zusammenhang zwischen der nicht-renalen Clearance von Meropenem und der Leberfunktion bei Patienten mit akutem Nierenversagen und gleichzeitiger Leberfunktionseinschränkung auf dem Boden einer Leberzirrhose (Giles et al. 2000).

\section{Meropenem und Alter}

Blumer et al. fanden bei 72 Kindern im Alter von 2 Monaten bis 12 Jahren, die mit einer Dosis von $20 \mathrm{mg} / \mathrm{kg}$ Meropenem behandelt wurden, keine alters- oder dosisbedingten Effekte auf die Pharmakokinetik von Meropenem. Die mittlere Halbwertszeit und das Verteilungsvolumen im Steady State lagen bei 1,1 Stunden bzw. 0,4 1/kg. Die Gesamtclearance lag bei 5,6 ml/min/ $\mathrm{kg}$ und die renale Clearance bei $2,5 \mathrm{ml} / \mathrm{min} / \mathrm{kg}$ (Blumer et al. 1995).

Bei Neugeboren waren die Verteilungsvolumina $(0,521 / \mathrm{kg})$ und die Halbwertszeiten $(5,48$ 7,81 h) deutlich erhöht (van den Anker et al. 1997).

Bei älteren gesunden Freiwilligen wurde eine Verminderung der totalen und der renalen Meropenem-Clearance gefunden. Daraus resultierten eine erhöhte Plasma-AUC und eine erhöhte Eliminationshalbwertszeit (Ljungberg und Nilsson-Ehle 1988).

Nierenfunktionsstörung, Infektion, Neutropenie, Hämofiltration, Dialyse und Patientenalter beeinflussen als voneinander abhängige und unabhängige Faktoren die Pharmakokinetik von Meropenem in entscheidendem Maße und sollten Anlass zur Überprüfung und ggf. Modifikation der Therapie geben. 


\section{Patienten, Material und Methoden}

\subsection{Ein- und Ausschlusskriterien der Patienten}

In die Studie wurden 25 stationäre Patienten der Abteilungen Hämatologie-Onkologie und der kardiologischen Intensivstation der Universitätsmedizin Göttingen eingeschlossen, die aufgrund der Zeichen einer bakteriellen Infektion mit dem Breitbandantibiotikum Meropenem behandelt wurden. Die Indikation zur Gabe des Antibiotikums wurde von den behandelnden Ärzten ausschließlich aufgrund der klinischen Erfordernisse unter Berücksichtigung der Art und Schwere der Infektion und des zu erwartenden Erregerspektrums unbeeinflusst vom Konzept der Studie gestellt.

Die Patienten wurden nach klinischen Zeichen und laborchemischen Parametern einer Infektion beurteilt. Nach einer umfassenden Aufklärung der Patienten bzw. bei beatmeten oder komatösen Patienten derer Angehörigen und Abgabe einer schriftlichen Einverständniserklärung, wurde die Entscheidung zur Aufnahme in die Studie getroffen. Als Ausschlusskriterien galten Alter unter 18 Jahre und Allergie gegen Beta-Lactam-Antibiotika.

Die Studie wurde von der Ethikkommission der Universität Göttingen genehmigt und folgt den Richtlinien der Deklaration von Helsinki.

\subsection{Versuchsanordnung und Ablauf}

\section{Verabreichung von Meropenem}

Allen Patienten wurde abhängig von den klinischen Erfordernissen und gültigen Dosierungsanweisungen normogrammgerecht 2- bis 3-mal täglich eine Dosis von 500 oder $1000 \mathrm{mg}$ Meropenem (Meronem ${ }^{\circledR}$, Zeneca) intravenös als Kurzinfusion verabreicht.

Meropenem für intravenöse Gabe liegt als steriles weißes Pulver in den Dosierungen 250, 500 oder $1000 \mathrm{mg}$ als Trihydrat vor. Es ist gemischt mit Natriumcarbonat (208 mg Natriumcarbonat pro Gramm Meropenem) für die Zusetzung von Lösungen. Die übliche Mischung mit $0,9 \%$ Natriumchloridlösung $\mathrm{zu}$ einem Gesamtvolumen von $50 \mathrm{ml}$ wurde bei allen Studienpatienten durchgeführt. Bei allen Patienten erfolgte die intravenöse Infusion über einen Zeitraum von durchschnittlich 23 (10-79) Minuten.

\section{Gewinnung des Untersuchungsmaterials}

Bei jedem Patienten wurden während eines der ersten Meropenem-Dosierungsintervalle 5-8 Blutproben á $8 \mathrm{ml}$ mit Hilfe von Heparinat-Monovetten entnommen. 
Bei Patienten ohne zentralen Zugang erfolgte die Blutabnahme durch Punktion einer der Infusionsstelle kontralateral liegenden Armvene. Bei Patienten mit zentralem Zugang (Cubitalvenen-Katheter, Subclavia-Katheter, Jugularis-interna-Katheter) oder arteriellem Zugang (Radialis-Katheter) wurden zunächst 7-10 ml Blut vorgezogen und verworfen. Dadurch wurden diverse Infusionslösungen aus dem Kathetersystem entfernt und eventuelle Rückstände von Meropenem, die sich durch Adhäsion an den Katheterwänden befanden, minimiert. Nach der Probengewinnung wurde der entsprechende Zugang mit $10 \mathrm{ml} \mathrm{NaCl-}$ Lösung nachgespült, um Verunreinigungen im Kathetersystem zu vermeiden.

Die erste Blutentnahme erfolgte unmittelbar vor Beginn der Infusion von Meropenem (Minimumkonzentration des vorhergehenden Infusionsintervalls). Danach folgte die MeropenemInfusion. Innerhalb von 10 Minuten nach Ende der Infusion erfolgte die zweite Blutentnahme zur Bestimmung der Maximumkonzentration. Weitere Blutentnahmen folgten ca. 30 Minuten, 1, 2, 5, 6 Stunden nach Ende der Meropenem-Infusion und unmittelbar vor Beginn der nächsten Infusion von Meropenem. Bei Erstgabe des Antibiotikums wurde auf die Blutentnahme vor Beginn der Infusion verzichtet. Bei Verlängerung des Dosierungsintervalls auf 12 Stunden erfolgten zusätzliche Blutentnahmen nach 8 und 10 Stunden.

\section{Verarbeitung und Lagerung der Proben}

Zur Trennung von Plasma und zellulären Bestandteilen wurden die Blutproben innerhalb von 20 Minuten nach Gewinnung zentrifugiert (Frequenz 12.000/min über 10 Minuten). Die Plasmaproben wurden bis zur Bestimmung der Meropenem-Plasmakonzentration bei $-75{ }^{\circ} \mathrm{C}$ in Eppendorf-Cups asserviert.

\subsection{Bestimmung der Antibiotikakonzentrationen mittels HPLC}

Die Plasmakonzentrationen von Meropenem wurden im Rahmen einer assoziierten Doktorarbeit ermittelt. Die Messungen erfolgten mittels reverse-phase HPLC (high performance liquid chromatography). Die mobile Phase war zusammengesetzt aus 10,5\% Acetonitril und 20 mmol/l Ammoniumformiat als Phosphatpuffer (pH 4).

\section{Chromatographisches System:}

Für die Chromatographie kam das PC-gesteuerte HPLC-System LC 10A der Firma Shimadzu (Kyoto, Japan) zum Einsatz. Dieses bestand aus folgenden Komponenten:

- Controller CBM-10A 
- Pumpe LC-10AS

- Autoinjektor SIL-10A mit variablem Injektionsvolumen

- Säulenofen CTO-10A

- UV/Vis-Detektor SPD-10AV mit variabler Wellenlänge.

Die Integrations- und Steuersoftware des Systems war CLASS LC 10 Version 1.61.

Chromatographische Bedingungen:

Für die Trennung sorgte wiederum die Säule Synergi Polar-RP $4 \mu \mathrm{m}$, eine 250 x 4,60 mm Reversed-Phase- $\mathrm{C}_{18}$-Säule von Phenomenex. Ein Liter wurde folgendermaßen angesetzt: $\mathrm{Zu}$ etwa $800 \mathrm{ml}$ destilliertem Wasser wurden $20 \mathrm{mmol} / 1$ Ammoniumformiat (1,26 g/l) gegeben und daraufhin mit verdünnter Ameisensäure (ca.10 \%) der pH-Wert von 4 eingestellt. Dann wurden die in einem separaten Gefäß abgemessenen $105 \mathrm{ml}$ Acetonitril hinzugefügt. Schließlich wurde mit destilliertem Wasser das Volumen auf einen Liter aufgefüllt und zuletzt das Laufmittel im Vakuum für 5 Minuten entgast.

Der Flow betrug bei diesem Verfahren $1,1 \mathrm{ml} / \mathrm{min}$ bei einer Temperatur von $40^{\circ} \mathrm{C}$ im Säulenofen. Das Injektionsvolumen einer Probe betrug $50 \mu$ l. Es wurde die UV-Absorption bei 297 nm gemessen, was dem Absorptionsmaximum von Meropenem entsprach.

Probenaufbereitung:

Probenfällung zur Bestimmung der Gesamtkonzentration:

Zunächst wurde das Methanol, dem bereits der Interne Standard Ceftazidim in einer Konzentration von $50 \mathrm{mg} / 1$ zugesetzt und das in 10-ml-Aliquots eingefroren worden war, nach dem Auftauen mit $250 \mu \mathrm{l}$ Phosphorsäure (6 \%) versetzt und dann gründlich gevortext. Zu einem Plasma-Aliquot von $200 \mu \mathrm{l}$ in einem 1,5-ml-Reagiergefäß wurde dann das doppelte Probenvolumen dieser Methanollösung, also $400 \mu$, zugegeben. Dieses wurde daraufhin ca. 8 Sekunden auf einem Vortex-Mixer gemischt und dann 15 Minuten bei Raumtemperatur stehen gelassen. Danach wurde das Gemisch erneut gevortext und dann für 10 Minuten bei 14000 U/min zentrifugiert. Schließlich wurde der Überstand in ein 0,5-ml-Reagiergefäß abpipettiert und auf die Säule injiziert.

Retentionszeiten:

Meropenem: 5,6 Minuten

Ceftazidim (Interner Standard): 6,8 Minuten

Linearität:

Im Konzentrationsbereich zwischen 0,1 und $500 \mathrm{mg} / \mathrm{l}$ nachgewiesen.

Korrelationskoeffizient: größer als 0,99 


\section{Recovery:}

durchschnittlich $92,7 \%$

Nachweis- und Bestimmungsgrenze:

Nachweisgrenze: $0,075 \mathrm{mg} / \mathrm{l}$

Bestimmungsgrenze: $0,1 \mathrm{mg} / 1$

Präzision:

Präzision in der Serie: Variationskoeffizient (Vk) unter $2 \%$

Präzision von Tag zu Tag: Vk bei verschiedenen Konzentrationen zwischen 1,9 und 5,4 \%

\subsection{Pharmakokinetische Parameter}

Zur Bestimmung der individuellen pharmakokinetischen Parameter wurden die gemessenen Meropenem-Serumkonzentrations- versus Zeit-Daten mit Hilfe kommerziell erwerbbarer Pharmakokinetik-Software (NONMEM ${ }^{\circledR}$, GloboMax LLC, USA) verschiedenen pharmakokinetischen Modellen (1-, 2- und 3-Kompartment-Modell) angepasst. Das biexponentielle offene 2-Kompartment-Modell beschreibt die Pharmakokinetik von Meropenem am besten.

\subsection{Beurteilung der Schwere der Infektion}

Der Nachweis einer Infektion wurde aufgrund des klinischen Bildes, verschiedener bildgebender und labortechnischer Verfahren sowie dem kulturellen Nachweis der Mikroorganismen erbracht. Es lassen sich verschiedene Kategorien zur Verifizierung der Infektionsdiagnose unterscheiden:

FUO Fever of unknown origin

CDI Clinically defined infection

MDI Microbiologically defined infection

MCDI Microbilogically and clinically defined infection.

Klinische Befunde:

Bei jedem Patienten erfolgte eine umfangreiche allgemeine und spezielle klinische Untersuchung mit besonderer Beachtung folgender Befunde:

Zeichen der Infektion und Ausmaß der systemischen Reaktion (Fieber, Herzfrequenz, Atemfrequenz, RRsyst., Schüttelfrost, Diurese usw.), Zeichen von Verteilungsstörungen (Herzinsuffizienz, Niereninsuffizienz, Aszites, Ödeme), Zeichen von Organfunktions- 
störungen (Leber-, Niereninsuffizienz, respiratorische Insuffizienz), Ausscheidungsstörungen (z.B. Oligo-Anurie), mentaler Status (Somnolenz, Koma).

Apparative Diagnostik:

Alle vorhandenen apparativen Befunde mit Fokus auf Infektsuche wie Rö-Thorax, SonoAbdomen, Herz-ECHO, CT-Thorax oder Abdomen wurden zur klinischen Beurteilung der Patienten mit herangezogen.

Laborchemische Befunde:

Bei jedem Patienten wurde ein umfassender laborchemischer Status erhoben. Dieser Status beinhaltet hämatologische Parameter (Hämoglobin, Hämatokrit, Thrombozyten, Leukozyten), biochemische Werte (AST, ALT, yGT, AP, Bilirubin), Elektrolyte (Na, K, Ca), Nierenretentionswerte (Krea), Gerinnungsparameter (Quick, PTT, Fibrinogen, AT III) und CRP. Bei einigen Patienten wurde darüberhinaus eine Blutgasanalyse mit Säure-Basen-Status und Lactatbestimmung durchgeführt.

\section{Mikrobiologie:}

Alle während des stationären Aufenthalts erhobenen mikrobiologischen Befunde wurden dokumentiert und auf ihre Plausibilität und ihren Zusammenhang mit der aktuellen Infektsituation geprüft. Bei zeitlichem und kausalem Zusammenhang wurde neben dem kulturellen Befund und der Resistenzbestimmung ein quantitatives Antibiogramm angefertigt.

\section{Klassifizierung nach Schweregrad der Infektion (allgemeine Definition)}

Die Schwere der Infektion wurde nach den Kriterien der ACCP und CCM Consensus Conference Kriterien ermittelt (Bone et al. 1992).

SIRS: Als SIRS (systemic inflammatory response syndrom) bezeichnet man das Auftreten von mindestens zwei der folgenden klinischen Manifestationen, die aber auch unabhängig von einer Infektion auftreten können:

- Körpertemperatur $>38^{\circ} \mathrm{C}$ oder $<36^{\circ} \mathrm{C}$

- Herzfrequenz $>90 / \mathrm{min}$

- Tachypnoe mit einer Frequenz von $>20 /$ min oder Hyperventilation mit einem $\mathrm{PaCO}_{2}$ $<32 \mathrm{mmHg}$ 
- Anstieg der Leukozyten auf $>12.000 / \mu l$ oder Abfall auf $<4000 / \mu l$ oder eine Linksverschiebung im Differentialblutbild (Anstieg der stabkernigen Granulozyten) auf $>10 \%$.

(Definitionsgemäß sollten diese pathologischen Abweichungen unabhängig von Chemotherapie-induzierter Neutropenie oder Leukopenie auftreten)

SIRS kann mit einer großen Zahl von klinischen Konditionen assoziiert sein. Neben Infektionen können auch nicht-infektiöse Ursachen zugrunde liegen, wie Pankreatitis, Multitrauma oder Gewebsverletzungen, hämorrhagischer Schock, autoimmunologische Erkrankungen oder die Zufuhr exogener Substanzen (Medikamente, Toxine usw.), die eine Zytokinfreisetzung hervorrufen.

Sepsis: Sepsis ist SIRS als Folge von Infektion.

Sepsis und ihre Folgen repräsentieren ein Kontinuum von klinischen und pathophysiologischen Schweregraden.

Schwere Sepsis: Schwere Sepsis (severe sepsis) ist definiert als Sepsis mit Organdysfunktion, Hypoperfusion oder sepsisinduzierter Hypotension.

Hypoperfusion umfasst Lactatazidose, Oligurie und akuter Abfall des mentalen Status (Glasgow Coma Scale). Als sepsis-induzierte Hypotension bezeichnet man einen Abfall des systolischen Blutdrucks auf unter $90 \mathrm{mmHg}$ oder einen Abfall von der Baseline um mindestens $40 \mathrm{mmHg}$.

Septischer Schock: Septischer Schock umfasst schwere Sepsis mit sepsisinduzierter Hypotension oder die ausschließliche Aufrechterhaltung eines normalen Blutdrucks durch den Einsatz von positiv inotropen Medikamenten, die Notwendigkeit einer forcierten Flüssigkeitszufuhr und das Vorhandensein von Hypoperfusion mit Organdysfunktion.

\section{$\underline{\text { Klassifizierung der Intensivpatienten nach dem Schweregrad der Infektion }}$}

Die Intensivpatienten wurden nach Art und Schwere der Infektion in verschiedene Gruppen unterteilt:

1) keine Sepsis: weniger als 2 SIRS - Kriterien

2) wahrscheinliche Sepsis:

Fieber mit mindestens 2 SIRS Kriterien und Nachweis von Keimen in sterilen Kompartimenten oder positiven röntgenologischen Pneumonie-Nachweis 
3) Sepsis: mindestens 2 SIRS Kriterien und mikrobiologischer Nachweis einer systemischen Infektion (positive Blutkultur oder positive bronchoalveoläre Lavage (BAL)

4) schwere Sepsis/septischer Schock: Patienten mit septischem Schock hatten über die Sepsiskriterien hinaus eine Organdysfunktion (z.B. Schocklunge oder akutes Nierenversagen), eine Hypoperfusion mit Lactatacidose, Oligurie oder Abfall des mentalen Status und eine sepsisinduzierte Hypotension mit Abfall des systolischen Blutdrucks um $40 \mathrm{mmHg}$ von der Baseline oder einem Blutdruckabfall auf unter $90 \mathrm{mmHg}$ oder Aufrechterhaltung eines Blutdrucks über 90 $\mathrm{mmHg}$ durch Einsatz positiv inotroper Substanzen oder forcierter Flüssigkeitszufuhr.

\section{$\underline{\text { Klassifizierung der hämatoonkologischen Patienten nach Schweregrad der Infektion }}$}

Die klassischen Sepsis-Kriterien, die den Grad der systemischen Reaktion angeben sind bei hämatoonkologischen Patienten häufig nicht anwendbar.

Bei hämatoonkologischen Patienten ist Fieber oft das einzige Zeichen einer Sepsis. Die Anzahl der Leukozyten spiegelt nur in geringem Maß eine Reaktion auf eine mikrobielle Infektion wider. Sie ist oft Ausdruck der zytostatischen Therapie oder des Progresses der neoplastischen Erkrankung. Neutropenie oder Blastenschub sind zwar prognostische Faktoren für eine Infektion, aber nicht deren Folge.

Tachykardie und Tachypnoe bei febrilen hämatoonkologischen Patienten lassen sich nicht eindeutig auf eine systemische Reaktion zurückführen, sondern können auch Ausdruck einer Anämie sein.

Eine negative Blutkultur ist kein Ausschlusskriterium für Sepsis.

Aus diesem Grund erfolgt in dieser Gruppe die Klassifikation der Schwere der Infektion nach folgender Definition:

1) keine Sepsis: Fieber ohne Neutropenie und ohne kulturellen Nachweis von Keimen in sterilen Kompartimenten oder positiven röntgenologischen Pneumonie-Nachweis

2) wahrscheinliche Sepsis: Fieber in Neutropenie oder Fieber mit mindestens 2 SIRS Kriterien und Nachweis von Keimen in sterilen Kompartimenten oder positiven röntgenologischen Pneumonie-Nachweis

3) Sepsis: Fieber mit mindestens 2 SIRS- Kriterien und positiver Blutkultur. 
Unter den hämatoonkologischen Patienten waren keine Patienten mit schwerer Sepsis oder septischem Schock.

\section{Berechnung der Kreatinin-Clearance}

Die Berechnung der Kreatinin-Clearance erfolgte nach der Formel von Cockroft und Gault. Die so errechnete Kreatinin-Clearance ist abhängig von Größe, Alter, Geschlecht und Kreatininspiegel im Plasma.

$\mathrm{M}: \quad \mathrm{Cl}_{\mathrm{krea}}=((140-$ Alter $) * \mathrm{IBW}) /(\mathrm{P}-$ Kreatinin $* 72)$
$\mathrm{W}: \mathrm{Cl}_{\mathrm{krea}}=0,85 *((140-$ Alter $) * \mathrm{IBW}) /(\mathrm{P}-$ Kreatinin $* 72)$

M: IBW $=50+2,3 *((\mathrm{Größe} / 2,24)-60)$

$\mathrm{W}: \mathrm{IBW}=45,5+2,3 *((\mathrm{Größe} / 2,24)-60)$

$($ IBW = ideal body weight $)$

Dabei ist zu beachten, dass das Serumkreatinin kein Schätzmaß für die Nierenfunktion bei folgenden pathophysiologischen Zuständen ist:

- $\quad$ akutem Nierenversagen

- Therapie des akuten Nierenversagens

- Hämodialyse

- jeder anderen pathophysiologischen Situation, die mit einer instabilen Nierenfunktion einhergeht.

Berechnung der glomerulären Filtrationsrate (GFR)

Die Abschätzung der glomerulären Filtrationsrate erfolgte nach der MDRD-Formel.

Die eGFR (geschätzte glomeruläre Filtrationsrate) ist abhängig von Alter und Serumkreatinin.

eGFR (männlich) $=175 \cdot$ P-Kreatinin $^{-1,154} \cdot$ Alter in Jahren $^{-0,203}$

$\mathrm{eGFR}($ weiblich $)=175 \cdot$ P-Kreatinin $^{-1,154} \cdot$ Alter in Jahren $^{-0,203} \cdot 0,742$ 


\subsection{Beurteilung des Outcome der Patienten}

Der Outcome der Patienten lässt sich sowohl klinisch als auch mikrobiologisch beurteilen. Aufgrund der geringen Spezifität der mikrobiologischen Befunde, insbesondere bei neutropenischen Patienten, ist deren Aussagekraft bei der Beurteilung des Outcome eingeschränkt.

Der klinische Outcome der Patienten und insbesondere der Fieberverlauf sind aussagekräftiger bei der Beurteilung der infektiologischen Lage der Patienten.

\begin{tabular}{|c|c|}
\hline gut & $\begin{array}{l}\text { Entfieberung unter Meropenem durch erfolgreiche antimikrobielle Therapie } \\
\text { innerhalb von } \leq 3 \text { Tagen für mindestens } 7 \text { Tage }^{1}\end{array}$ \\
\hline befriedigend & $\begin{array}{l}\text { Entfieberung unter Meropenem durch erfolgreiche antimikrobielle Therapie } \\
\text { nach }>3 \text { Tagen oder Wiederauftreten von Fieber innerhalb von } 7 \text { Tagen nach } \\
\text { Absetzen der Antibiose }^{2}\end{array}$ \\
\hline unbefriedigend & $\begin{array}{l}\text { keine Entfieberung unter Meropenem, aber Ab- oder Umsetzen der } \\
\text { antimikrobiellen Therapie innerhalb von }<3 \text { Tagen }^{3}\end{array}$ \\
\hline mangelhaft & keine Entfieberung unter Meropenem trotz Therapie über mehr als 3 Tage \\
\hline ungenügend & keine Entfieberung unter Meropenem; Tod durch Infektion \\
\hline
\end{tabular}

Tabelle 1: Outcome-Klassifikation unter Meropenem-Therapie

${ }^{1}$ ein erneutes Auftreten von Fieber nach $>7$ Tagen ist als Reinfektion zu werten und nicht auf eine ineffektive Therapie der Primärinfektion zurückzuführen

${ }^{2}$ das erneute Auftreten von Fieber innerhalb von $\leq 7$ Tagen spricht für die Persistenz der primären Infektion

${ }^{3}$ es ist davon auszugehen, dass innerhalb von 3 Tagen trotz eventueller Empfindlichkeit des Keimes eine vollständige Eradikation nicht erreicht werden konnte

\subsection{Statistische Methoden}

Die statistische Auswertung erfolgte mit Hilfe deskriptiver Kenngrößen wie Mittelwerten, Standardabweichungen, Median, Range, Korrelationskoeffizient R und Bestimmtheitsmaß R². Als statistische Tests kam der unverbundene T-Test mit statistischer Signifikanz $p<0,05$ zur Anwendung. 


\section{$3 \quad$ Ergebnisse}

\subsection{Demographische Daten}

In die Studie wurden 25 Patienten aufgenommen, die sich im Zeitraum von April 2000 bis August 2001 in stationärer Behandlung der hämatoonkologischen Normalstation oder der kardiologischen Intensivstation des Klinikums der Georg-August-Universität Göttingen befanden und aufgrund einer Infektion mit dem Antibiotikum Meropenem behandelt wurden.

Unter den Patienten waren 11 Männer und 14 Frauen im Alter zwischen 33 und 85 Jahren. Die durchschnittliche Größe der Patienten lag bei $170 \mathrm{~cm}$, ihr durchschnittliches Gewicht betrug $78,3 \mathrm{~kg}$.

Unter den Studienteilnehmern befanden sich 10 Patienten mit malignen hämatologischen Erkrankungen und 15 Intensivpatienten mit verschiedenen anderen Grunderkrankungen.

Einen Überblick über die demographischen Daten gibt Tabelle 2. Detaillierte Angaben aller Haupt- und Nebendiagnosen finden sich in den Tabellen 7, 14 und 15 (Anhang).

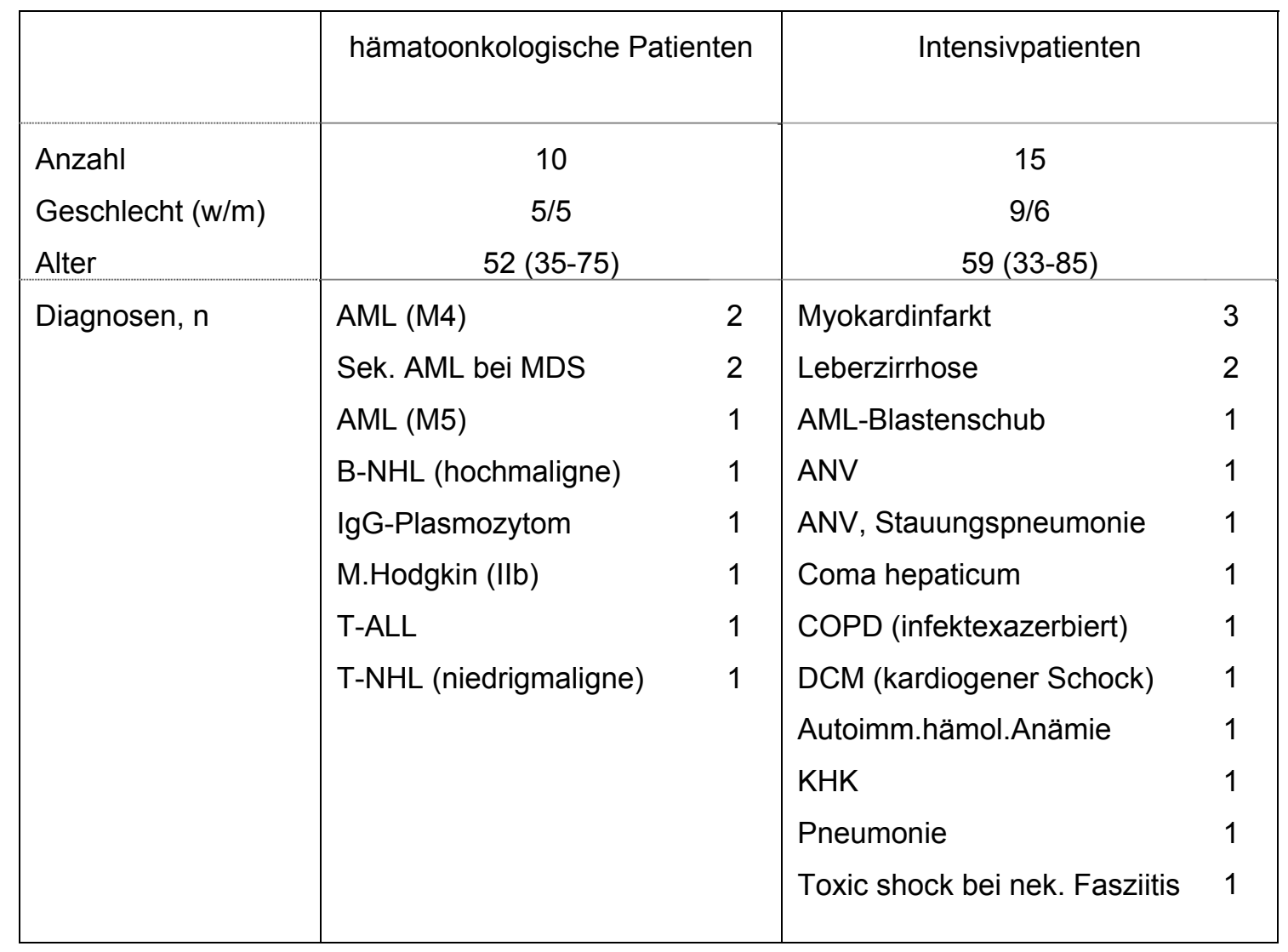

Tabelle 2: Demographische Daten 


\subsection{Hämatoonkologische Patienten}

\subsubsection{Demographische Daten der hämatoonkologischen Patienten}

Unter den Patienten mit maligner hämatologischer Erkrankung waren 5 Männer und 5 Frauen im Alter zwischen 35 und 75 Jahren (Median/ \pm SD; $55 / \pm 11,8$ Jahre). Die Durchschnittsgröße lag bei $168 \mathrm{~cm}(160-175 \mathrm{~cm})$, das durchschnittliche Gewicht betrug $72 \mathrm{~kg}(48,0-85,1 \mathrm{~kg})$.

Unter den Patienten befanden sich 6 Neutropeniker (Anzahl der neutrophilen Granulozyten im Differentialblutbild $<1000 / \mu 1$ ) und 4 Nicht-Neutropeniker.

\begin{tabular}{|c|c|c|c|c|}
\hline & \multicolumn{2}{|c|}{ Neutropeniker $(n=6)$} & \multicolumn{2}{|l|}{ Nicht-Neutropeniker $(n=4)$} \\
\hline 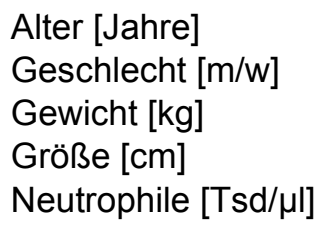 & $\begin{array}{c}44(35-53) \\
2 / 4 \\
72(48-85) \\
165(160-175 \\
0,14(0,05-0,62\end{array}$ & & $\begin{array}{c}60(47-75) \\
3 / 1 \\
71(65-75) \\
170(165-175) \\
2,12(1,62-5,87)\end{array}$ & \\
\hline Diagnosen,n & $\begin{array}{l}\text { T-NHL } \\
\text { B-NHL } \\
\text { AML } \\
\text { sek. AML } \\
\text { M.Hodgkin }\end{array}$ & $\begin{array}{l}1 \\
1 \\
2 \\
1 \\
1\end{array}$ & $\begin{array}{l}\text { T-NHL } \\
\text { lgG-Plasmozytom } \\
\text { AML } \\
\text { T-ALL }\end{array}$ & $\begin{array}{l}1 \\
1 \\
1 \\
1\end{array}$ \\
\hline
\end{tabular}

Tabelle 3: Demographische Daten der hämatoonkologischen Patienten

\subsubsection{Diagnosen und weitere Erkrankungen der hämatoonkologischen Patienten}

Siehe Tabelle 7. im Anhang

\subsubsection{Laborparameter der hämatoonkologischen Patienten}

Die laborchemischen Parameter: Elektrolyte, Protein, Albumin, Kreatinin, Transaminasen (AST, ALT), Cholestasewerte (AP, $\gamma \mathrm{GT}$, Bilirubin), hämatologische und hämostasiologische Befunde sind in Tabelle 8 im Anhang dokumentiert.

\subsubsection{Infektionsparameter der hämatoonkologischen Patienten}

In der Gruppe der hämatoonkologischen Patienten war bei 5 Patienten die Infektion sowohl klinisch als auch mikrobiologisch gesichert (CMDI). Bei 2 Patienten lagen lediglich klinische Zeichen einer Infektion vor (CDI). Bei 3 Patienten war Fieber der einzige Hinweis auf eine mögliche Infektion (FUO). Die Infektionsparameter der hämatoonkologischen Patienten sind in Tabelle 9 im Anhang zusammengefasst. 
Nach dem Schweregrad der Infektion lassen sich 3 Gruppen von Patienten unterscheiden (keine Sepsis, wahrscheinliche Sepsis, Sepsis). Sie lassen sich, wie in Tabelle 4 dargestellt, charakterisieren (die Unterscheidungskriterien finden sich auch im Methodenteil auf Seite 30):

\begin{tabular}{|l|l|l|}
\hline & Einschlusskriterien & Patienten Nr. \\
\hline 1) keine Sepsis & $\begin{array}{l}\text { Hämatoonkologische Patienten, bei denen } \\
\text { aufgrund des fehlenden Nachweises einer } \\
\text { schweren Infektion wahrscheinlich keine } \\
\text { Sepsis vorliegt. }\end{array}$ & $4,5,6$ \\
\hline $\begin{array}{c}\text { 2) wahrscheinliche } \\
\text { Sepsis }\end{array}$ & $\begin{array}{l}\text { Hämatoonkologische Patienten, bei denen } \\
\text { aufgrund der Zeichen einer schweren Infektion } \\
\text { trotz fehlenden mikrobiologischen Nachweises } \\
\text { einer systemischen Infektion eine Sepsis } \\
\text { wahrscheinlich ist. }\end{array}$ & $1,2,3$ und 9 \\
\hline 3) Sepsis & $\begin{array}{l}\text { Hämatoonkologische Patienten, bei denen } \\
\text { aufgrund der Zeichen einer schweren Infektion } \\
\text { mit Nachweis einer Bakteriämie eine Sepsis } \\
\text { vorliegt. }\end{array}$ & 7,8 und 10 \\
\hline
\end{tabular}

Tabelle 4: Schweregrad der Infektion bei hämatoonkologischen Patienten

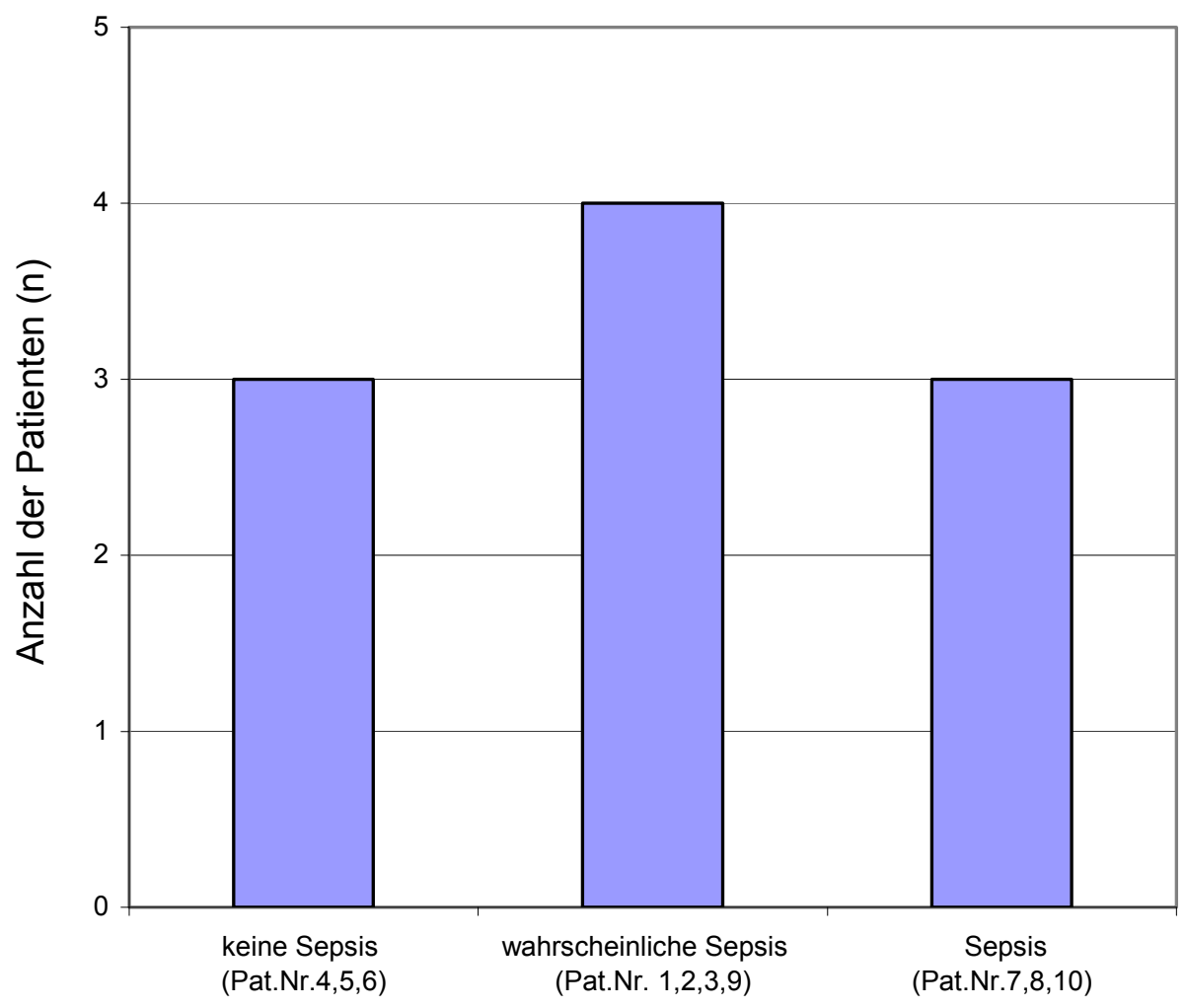

Abbildung 3: Hämatoonkologische Patienten unterteilt nach Schwere der Infektion 


\subsubsection{Pharmakokinetische Daten der hämatoonkologischen Patienten}

Alle Patienten mit hämatoonkologischer Grunderkrankung erhielten eine Dosis von $1000 \mathrm{mg}$ Meropenem alle 8 Stunden als iv. Kurzinfusion. Die Dauer der Infusion betrug 21 (10-32) Minuten.

Die nach Cockroft und Gault errechnete Kreatinin-Clearance [Mittelwert/Median (Range)] aller Patienten mit hämatoonkologischer Erkrankung lag bei 89,1/80,2 (55,6-126,7) ml/min.

Die nach der MDRD-Formel geschätzte glomeruläre Filtrationsrate bei den hämatoonkologischen Patienten betrug 90,2/81,2 (54,3-140,4) $\mathrm{ml} / \mathrm{min}$.

Die Meropenem-Maximumkonzentration lag bei 55,5/52,7 (38,2 - 72.7) mg/l, die Minimumkonzentration am Ende des Dosierungsintervalls bei 1,1/0,6 $(<0,1-2,8) \mathrm{mg} / \mathrm{l}$. Die gefundenen Maxima unterschieden sich somit um einen Faktor 1,9, die Minima um einen Faktor größer als 28. Es kam zu keiner Kumulation. Die Plasmakonzentrationsverläufe zeigt Abbildung 4.

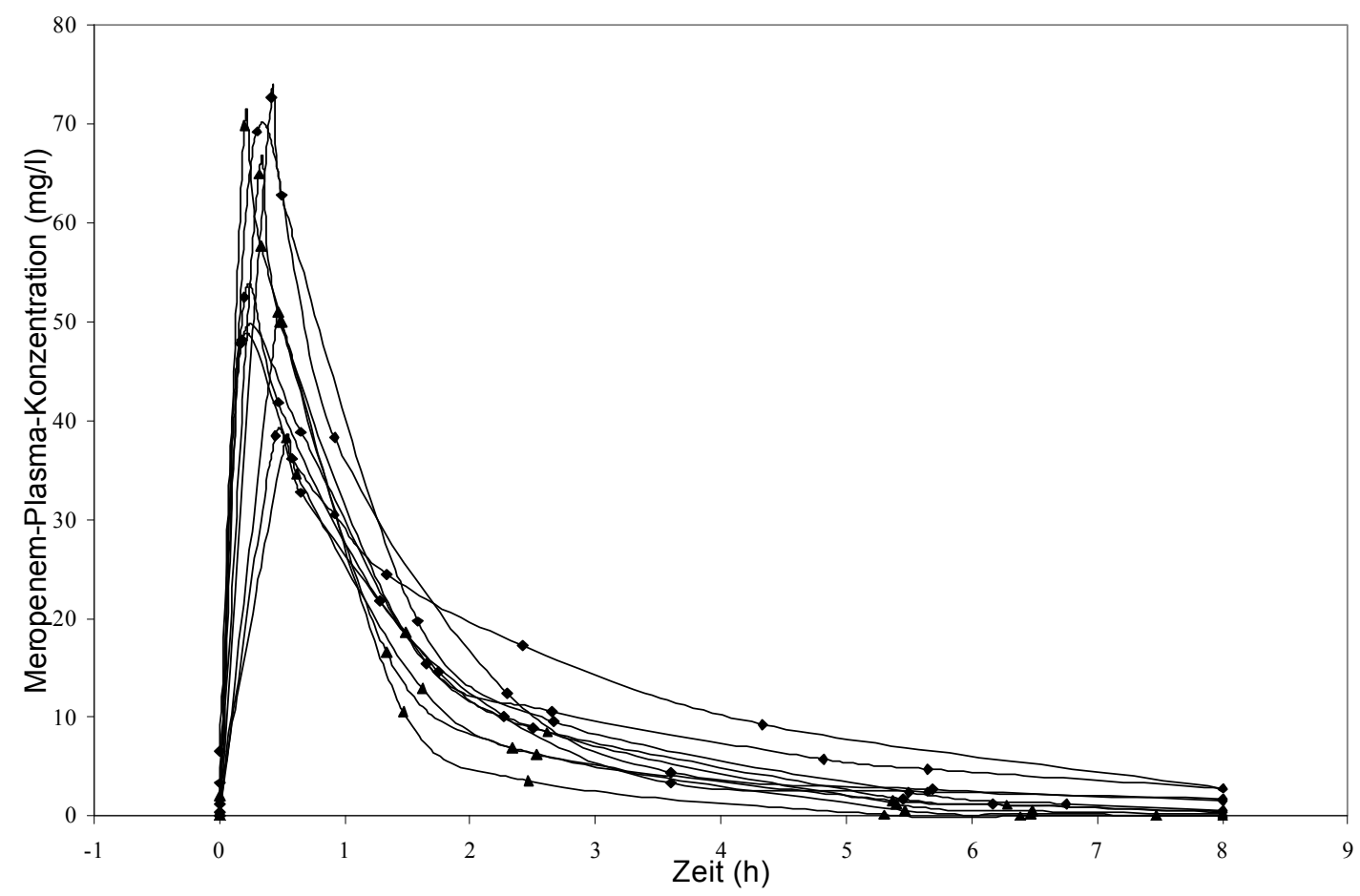

Abbildung 4: Plasmakonzentrationsverläufe der hämotoonkologischen Patienten

Das Verteilungsvolumen von Meropenem im Steady-State $\left(\mathrm{V}_{\mathrm{ss}}\right)$ lag bei 23,3/ 22,8 $(15,0-37,0)$ 1 bzw. 0,33/0,33 (0,18-0,53) 1/kg.

Die Halbwertszeit in der Verteilungsphase $\left(T_{1 / 2 \alpha}\right)$ lag bei 0,42/0,37 $(0,21-0,74)$ Stunden und in der Eliminationsphase $\left(\mathrm{T}_{1 / 2 \beta}\right)$ bei 1,97/1,41 $(0,75-4,77)$ Stunden. 
Die Fläche unter der Zeit-Konzentrations-Kurve vom Beginn der Infusion bis unendlich $\left(\mathrm{AUC}_{0-\infty}\right)$ lag bei 82,7/85,4 (48,3-120,3) $\mathrm{mg} / 1 \cdot \mathrm{h}$.

Die totale Plasmaclearance von Meropenem betrug 217,7/196,9 (138,6-345,3) $\mathrm{ml} / \mathrm{min}$.

Die MIC90 von Enterobacteriaceae (2 mg/l) wurde nach 6,29/5,95 (2,79-9,43) Stunden bzw. 75,1/74,3 (34,8-100,0) \% des Dosierungsintervalls von jeweils 8 Stunden unterschritten.

Die MIC90 von Pseudomonas aeruginosa (4 mg/l) wurde nach 4,53/4,24 (2,19-7,41) Stunden bzw. 56,7/53,0 (27,4-89,3) \% des Dosierungsintervalls unterschritten.

Unter den hämatoonkologischen Patienten bestand eine gute lineare Korrelation zwischen der Kreatinin-Clearance und der Meropenem-Plasmaclearance $(\mathrm{R}=0,78)$. Dies bedeutet, dass die Elimination von Meropenem in dieser Patientengruppe überwiegend von der Nierenfunktion bestimmt wurde.

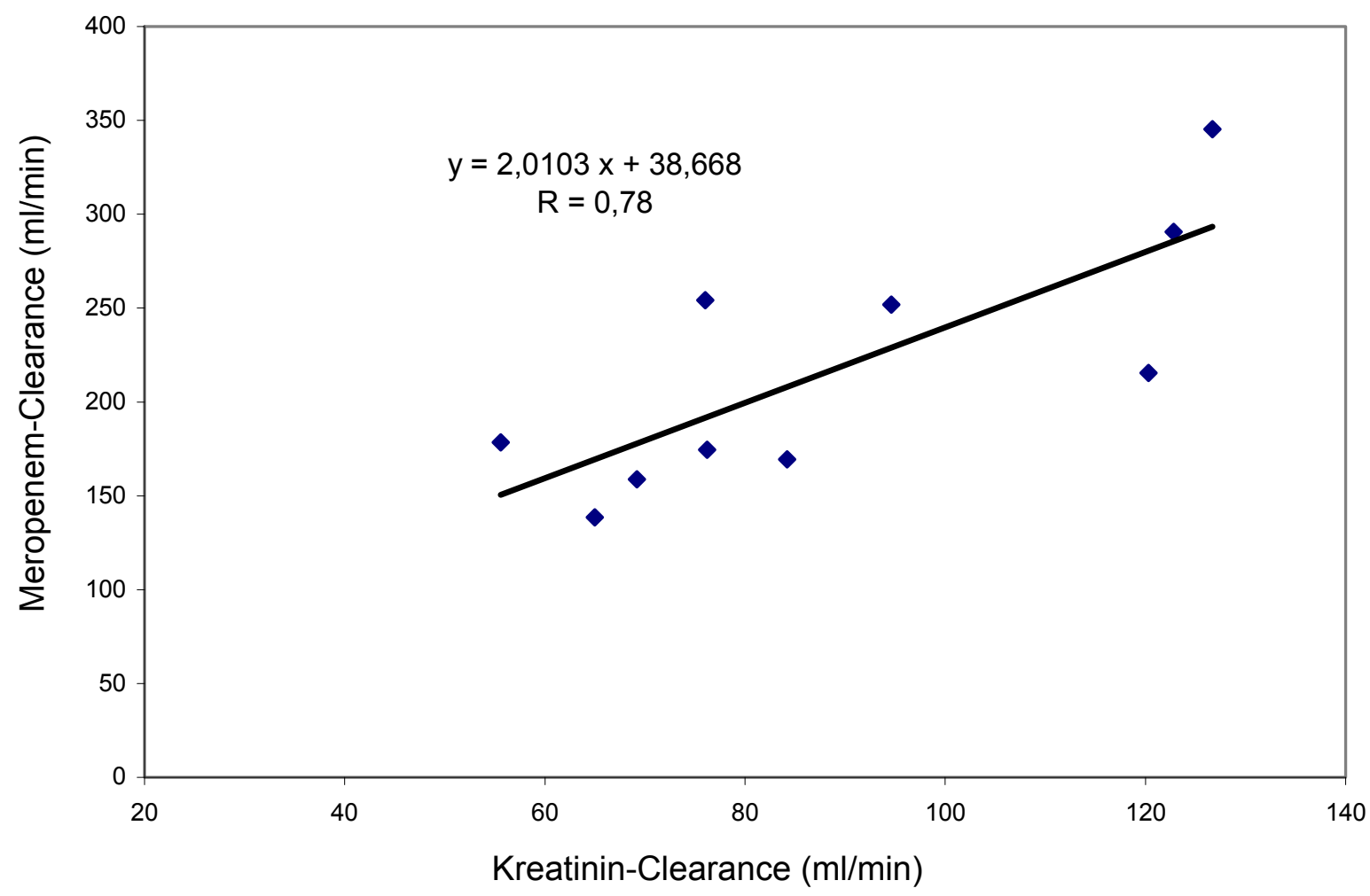

Abbildung 5: Korrelation zwischen Kreatinin- und Meropenem-Clearance bei den hämatoonkologischen Patienten

Die pharmakokinetischen Parameter der hämatoonkologischen Patienten sind in Tabelle 10 und $11 \mathrm{im}$ Anhang zusammengefasst. 


\subsubsection{Pharmakokinetische Daten der febrilen neutropenischen Patienten}

Es fiel auf, dass unter den von uns untersuchten hämatoonkologischen Patienten $(n=10)$ gerade die febrilen neutropenischen Patienten $(n=6)$ zu einer hochnormalen KreatininClearance tendierten und eine höhere Meropenem-Clearance aufwiesen als nichtneutropenische Patienten. Sie tendierten darüber hinaus zu kleineren Verteilungsvolumina. Die mittlere Aufenthaltsdauer des Pharmakons im Körper (MRT) war bei den Neutropenikern deutlich verkleinert. Die Neutropeniker hatten niedrigere Minimumkonzentrationen und unterschritten die geforderten Mindestkonzentrationen früher als die hämatoonkologischen Patienten ohne Neutropenie.

Es ist allerdings zu berücksichtigen, dass es sich nur um sehr kleine Patientengruppen handelt und die gefundenen Unterschiede sich nicht ohne weiteres verallgemeinern lassen.

Die nach Cockroft und Gault errechnete Kreatinin-Clearance der von uns untersuchten hämatoonkologischen Patienten betrug bei den Neutropenikern 102,8/107,5 (76,0-126,7) $\mathrm{ml} / \mathrm{min}$ und bei den Nicht-Neutropenikern 68,5/67,1 $(55,6-84,2) \mathrm{ml} / \mathrm{min}$. Bei den Neutropenikern war die Kreatinin-Clearance somit im Durchschnitt 50\% größer als bei den Nicht-Neutropenikern.

Die Meropenem-Maximumkonzentration [Mittelwert/Median (Range)] lag in der Gruppe der Nicht-Neutropenikern bei 54,4/50,4 $(47,8-69,3) \mathrm{mg} / \mathrm{l}$ und bei den Neutropenikern bei $56,2 / 58,9(38,2-72,7) \mathrm{mg} / 1$. Es bestand also kein wesentlicher Unterschied zwischen beiden Gruppen.

Wie oben beschrieben bestand bei den hämatoonkologischen Patienten ein linearer Zusammenhang zwischen der errechneten Kreatinin-Clearance und der totalen MeropenemPlasmaclearance. Es war demzufolge auch ein Zusammenhang zwischen Nierenfunktion und allen von der Meropenem-Clearance abhängigen Variablen $\left(\mathrm{C}_{\min }, \mathrm{T}_{1 / 2 \beta}, \mathrm{AUC}, \mathrm{T}<\mathrm{MIC}\right) \mathrm{zu}$ erwarten.

Die Minimumkonzentration betrug bei den Nicht-Neutropenikern [Mittelwert/Median (Range)] 2,3/2,3 (1,6-2,8) mg/l und bei den Neutropenikern 0,3/0,3 $(<0,1-0,7) \mathrm{mg} / \mathrm{l}$ und war in der erstgenannten Gruppe durchschnittlich 7,3-mal so groß. Es bestand ein signifikanter Unterschied zwischen beiden Gruppen $(p=0,0002)$. 


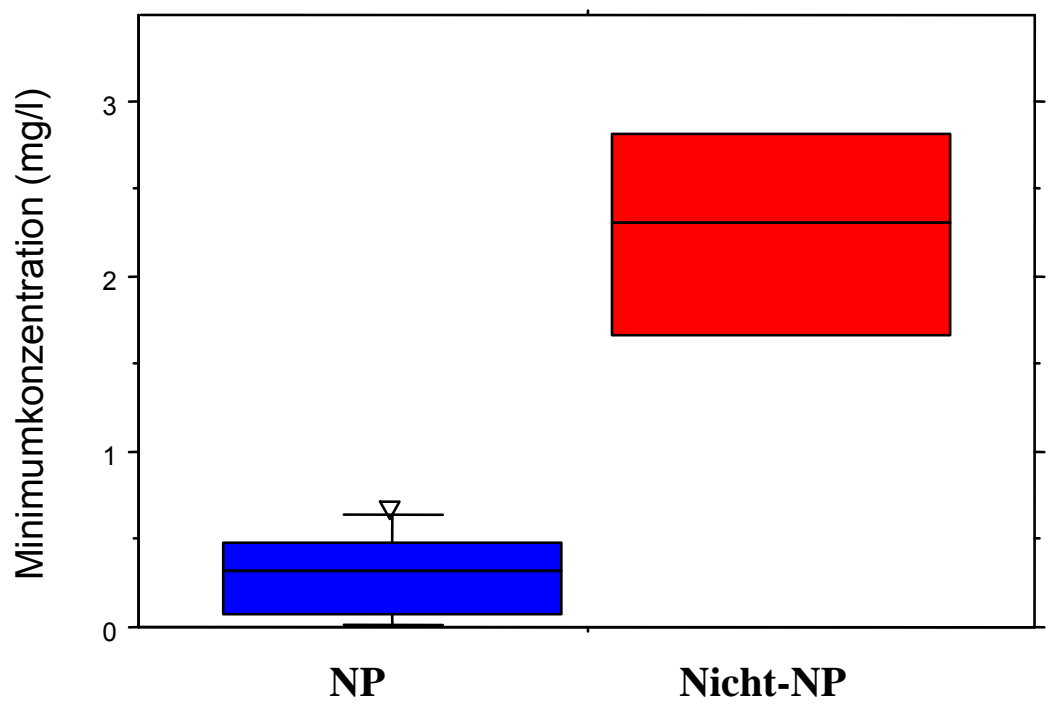

Abbildung 6: Meropenem-Minimumkonzentration der hämatoonkologischen Patienten unterteilt in neutropenische Patienten (NP) und nicht-neutropenische Patienten (Nicht-NP)

Die Gesamtclearance von Meropenem bei den Neutropenikern war mit [Mittelwert/Median (Range)] 255,3/253,1 (174,4-345,3) $\mathrm{ml} / \mathrm{min}$ durchschnittlich 58\% größer als bei den NichtNeutropenikern $(161,3 / 164,1(138,6-178,4) \mathrm{ml} / \mathrm{min})$. Der Unterschied zwischen beiden Gruppen war mit $\mathrm{p}=0,016$ signifikant.

Die Verteilungsvolumina für Meropenem im Steady-State lagen in der Gruppe der Nicht-NP bei 29,3/ 29,5 (21,1-37,0) 1 bzw. 0,42/0,41 (0,31-0,53) 1/kg und in der Gruppe der Neutropeniker bei 19,3/16,4 (15,0-27,3) 1 bzw. 0,27/0,28 (0,18-0,35) 1/kg. Die Verteilungsvolumina bei den Nicht-Neutropenikern waren durchschnittlich 51\% größer als bei den Neutropenikern. Auch hier bestand mit $\mathrm{p}=0,042$ ein signifikanter Unterschied zwischen beiden Gruppen.

Die Halbwertszeit in der Verteilungsphase $\left(T_{1 / 2 \alpha}\right)$ lag bei den Nicht-Neutropenikern bei $0,52 / 0,50(0,33-0,74) \mathrm{h}$ und bei den Neutropenikern bei $0,35 / 0,35(0,21-0,57) \mathrm{h}$. Die Halbwertszeit in der Eliminationsphase $\left(T_{1 / 2 \beta}\right)$ lag bei 3,23/3,00 $(2,14-4,77)$ Stunden für NichtNeutropeniker und 1,14/1,18 (0,75-1,43) Stunden für die Neutropeniker. Während die initialen Halbwertszeiten sich nur gering unterscheiden $(\mathrm{p}=0,146)$, bestand zwischen den terminalen Halbwertszeiten mit $\mathrm{p}=0,002$ ein signifikanter Unterschied.

Die Fläche unter der Zeit-Konzentrations-Kurve vom Beginn der Infusion bis unendlich $\left(\mathrm{AUC}_{0-\infty}\right)$ lag bei den Nicht-Neutropenikern bei 104,3/101,7 $(93,4-120,3) \mathrm{mg} /(1 \cdot \mathrm{h})$ und bei den neutropenischen Patienten bei 68,4/65,9 (48,3-95,5) $\mathrm{mg} /(1 \cdot \mathrm{h})$. Auch dieser Unterschied war mit $\mathrm{p}=0,006$ hoch signifikant. 
Neutropenische Patienten unterschritten die MIC90 von Pseudomonas aeruginosa (4 mg/l) bereits nach 49,1/52,1 (27,4-60,2) \% und die MIC90 von Enterobacteriaceae (2 mg/l) nach $65,7 / 67,9(34,8-82,0) \%$ des Dosierungsintervalls.

Nicht-Neutropeniker unterschritten die entsprechende Hemmkonzentration von Pseudomonas aeruginosa (4 mg/l) nach 68,0/68,1 (46,6-89,3) \% und die Hemmkonzentration von Enterobacteriaceae (2 mg/l) nach 89,3/99,5 (58,1-100) \% des Dosierungsintervalls.

Die minimale Hemmkonzentration für ausgesuchte Erreger wurde somit gerade von den neutropenischen Patienten oft frühzeitig unterschritten.

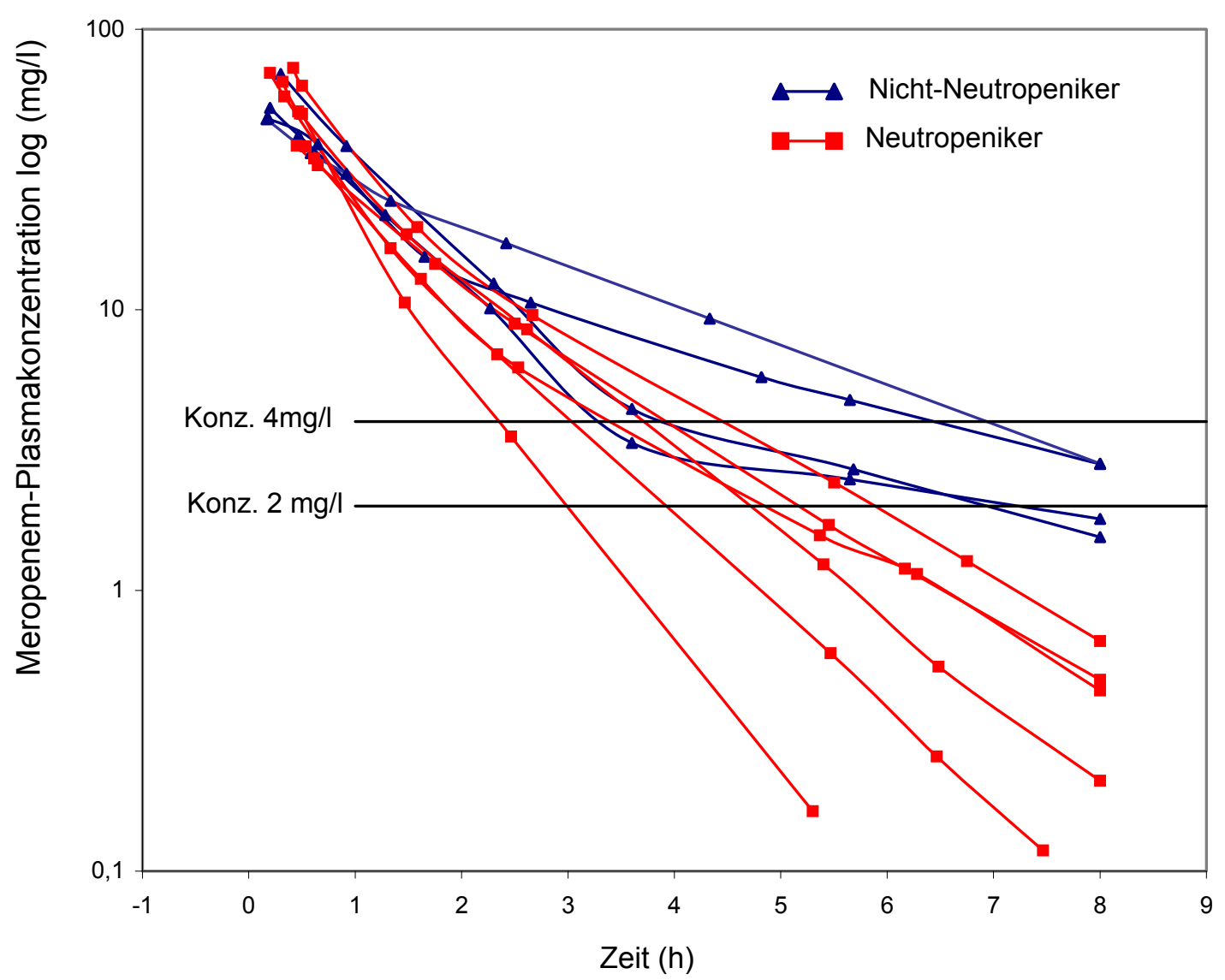

Abbildung 7: Zeit-Konzentrations-Verlauf (semilog.) und Unterschreitung der Hemmkonzentrationen 2 und $4 \mathrm{mg} / 1$

Nach dem Schweregrad der Infektionen ließ sich ein deutlicher Unterschied zwischen den Nicht-Neutropenikern und den Neutropenikern erkennen. Unter den Nicht-Neutropenikern hatten 3 Patienten nach aktuell gültiger Definition keine Sepsis. Bei einem Patienten bestand lediglich der Verdacht auf eine Sepsis.

Unter den neutropenischen Patienten waren 3 Patienten mit Sepsis und 3 Patienten mit Verdacht auf Sepsis. Die Neutropeniker hatten somit die schwereren Infektionen. 


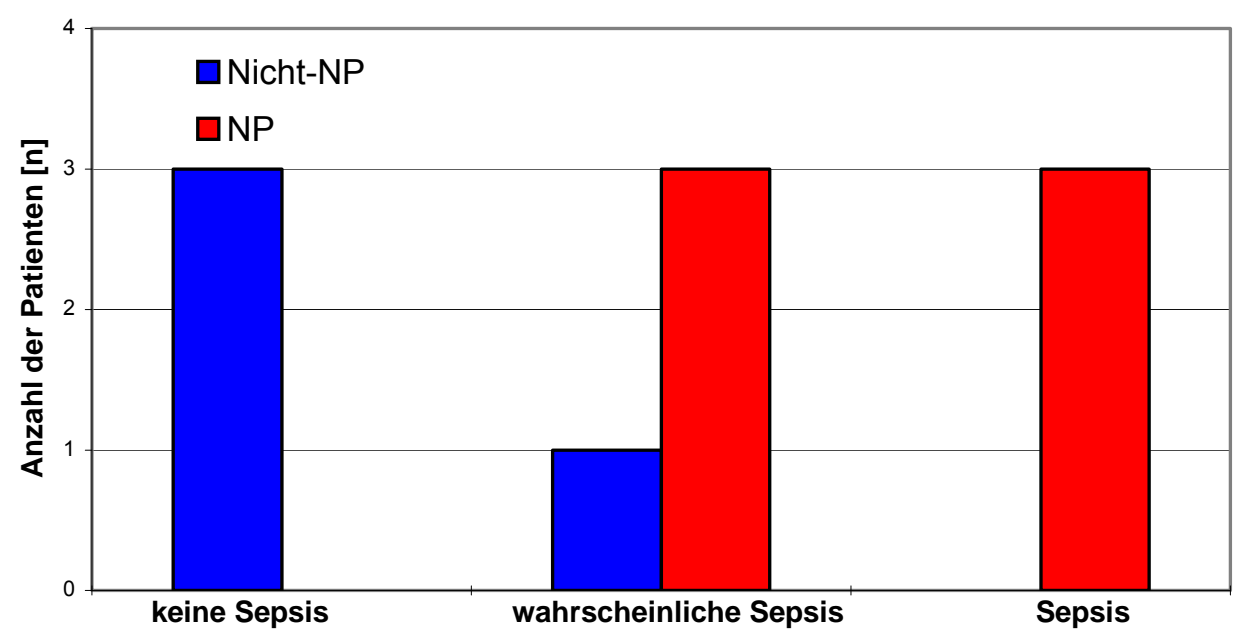

Abbildung 8: Anzahl der hämatoonkologischen Patienten mit verschiedenen Schweregraden der Infektion unterteilt in nicht-neutropenische und neutropenische Patienten

\subsubsection{Outcome der hämatoonkologischen Patienten}

In der Gruppe der Nicht-Neutropeniker hatten alle Patienten einen guten bis befriedigenden Outcome, dass heißt, bei allen Patienten kam es unter antimikrobieller Therapie mit Meropenem zu einer Entfieberung und einem Rückgang weiterer klinischer Zeichen einer Infektion für mindestens 7 Tage.

Unter den Neutropeniker hatten 4 Patienten ebenfalls einen guten bis befriedigenden Outcome, jedoch gab es hier einen Patienten, der nicht unter Meropenem entfieberte (Patient Nr.8) und einen Patienten, der an den Folgen der Infektion verstarb (Patient Nr.3).

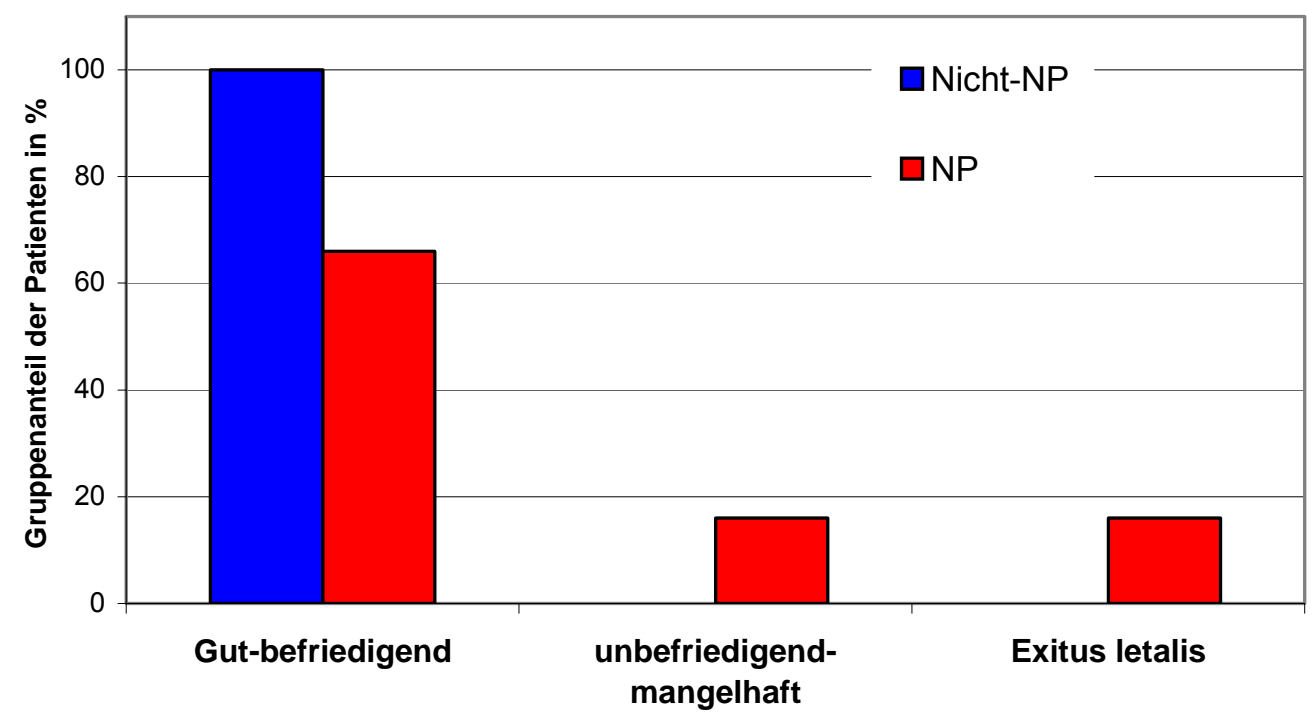

Abbildung 9: Outcome der hämatoonkologischen Patienten 
Da der Outcome nicht nur von der Effektivität der antibiotischen Therapie, sondern auch von der Empfindlichkeit des zu bekämpfenden Erregers, den Grunderkrankungen des Patienten und in besonderem Maße von Dauer und Schwere der Neutropenie abhängig ist und nur ein kleines Patientenkollektiv betrachtet wurde, ließ sich kein kausaler Zusammenhang zwischen Pharmakokinetik und Outcome herstellen.

Die Unterschiede der wichtigsten pharmakokinetischen Daten zwischen den von uns untersuchten neutropenischen und den nicht-neutropenischen Patienten sind zusammenfassend in den folgenden Box-Plots dargestellt.
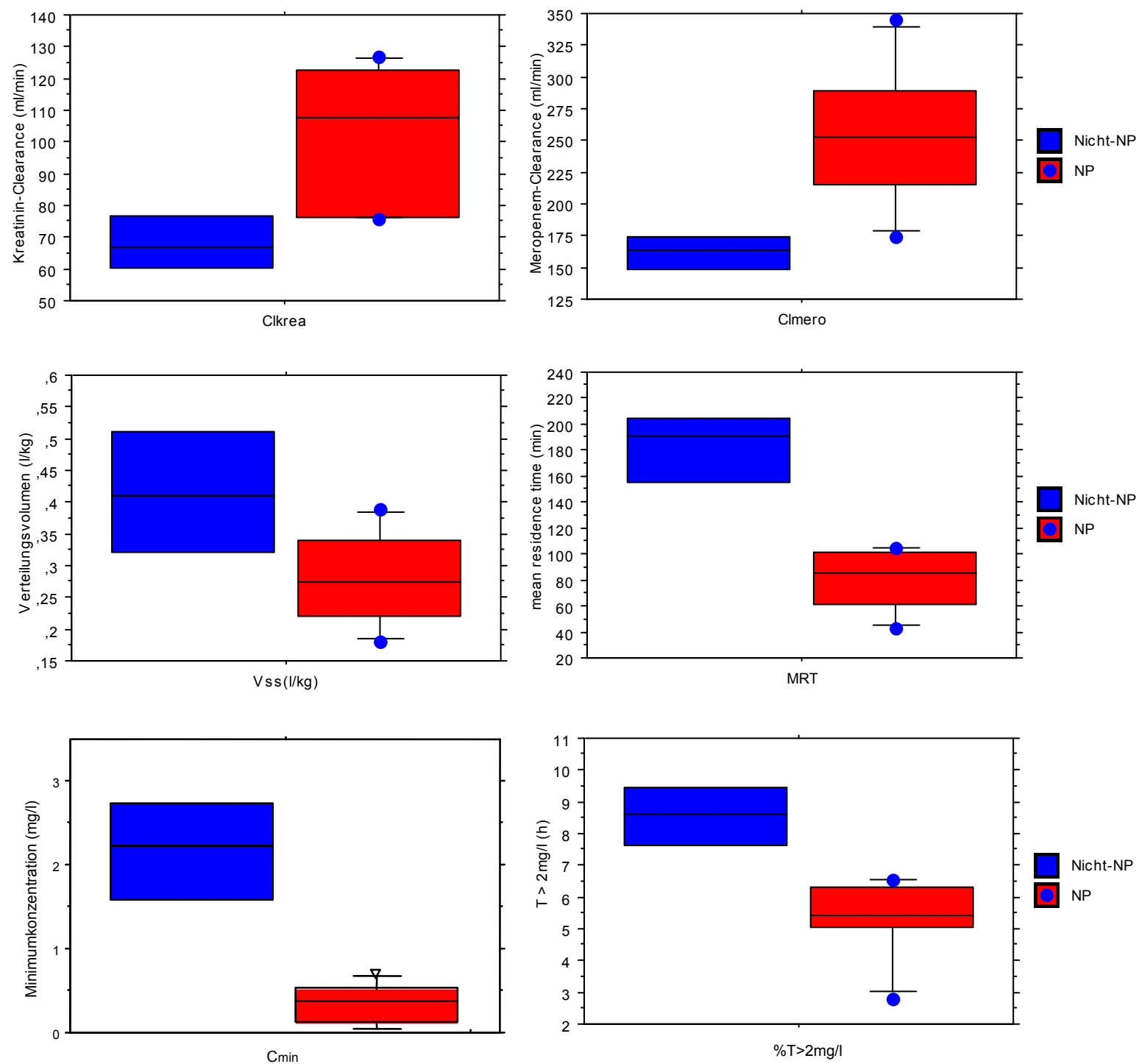

Abbildung 10: Unterschiede der wichtigsten pharmakokinetischen Parameter zwischen den neutropenischen (NP) und nicht-neutropenischen (Nicht-NP) hämatoonkologischen Patienten. 


\subsection{Intensivpatienten}

\subsubsection{Demographische Daten der Intensivpatienten}

Insgesamt wurden 15 Intensivpatienten in die Studie eingeschlossen. In dieser Patientengruppe waren 10 Männer und 5 Frauen im Alter von 33 bis 85 Jahren. Die durchschnittliche Größe lag bei 171 (155-182) cm, das durchschnittliche Gewicht betrug 82,3 (45,0-128,5) kg. Unter den Intensivpatienten waren 6 Patienten mit normaler Nierenfunktion (KreatininClearance $>60 \mathrm{ml} / \mathrm{min}$ ) und 9 Patienten mit eingeschränkter Nierenfunktion (KreatininClearance $<60 \mathrm{ml} / \mathrm{min}$ ), von denen 4 Patienten mit kontinuierlicher veno-venöser Hämofiltration (CVVHF) behandelt werden mussten.

Die Patienten hatten verschiedene Schweregrade einer Infektion. 1 Patient hatte eine Infektion ohne systemische Reaktion, 6 Patienten hatten eine Sepsis $(n=5)$ bzw. eine wahrscheinliche Sepsis $(n=1)$ und 8 Patienten hatten einen septischen Schock.

Einen Überblick über die demographischen Daten der Intensivpatienten zeigt Tabelle 5. Detaillierte Angaben über Haupt- und Nebendiagnosen finden sind in den Tabellen 14 und 15 im Anhang.

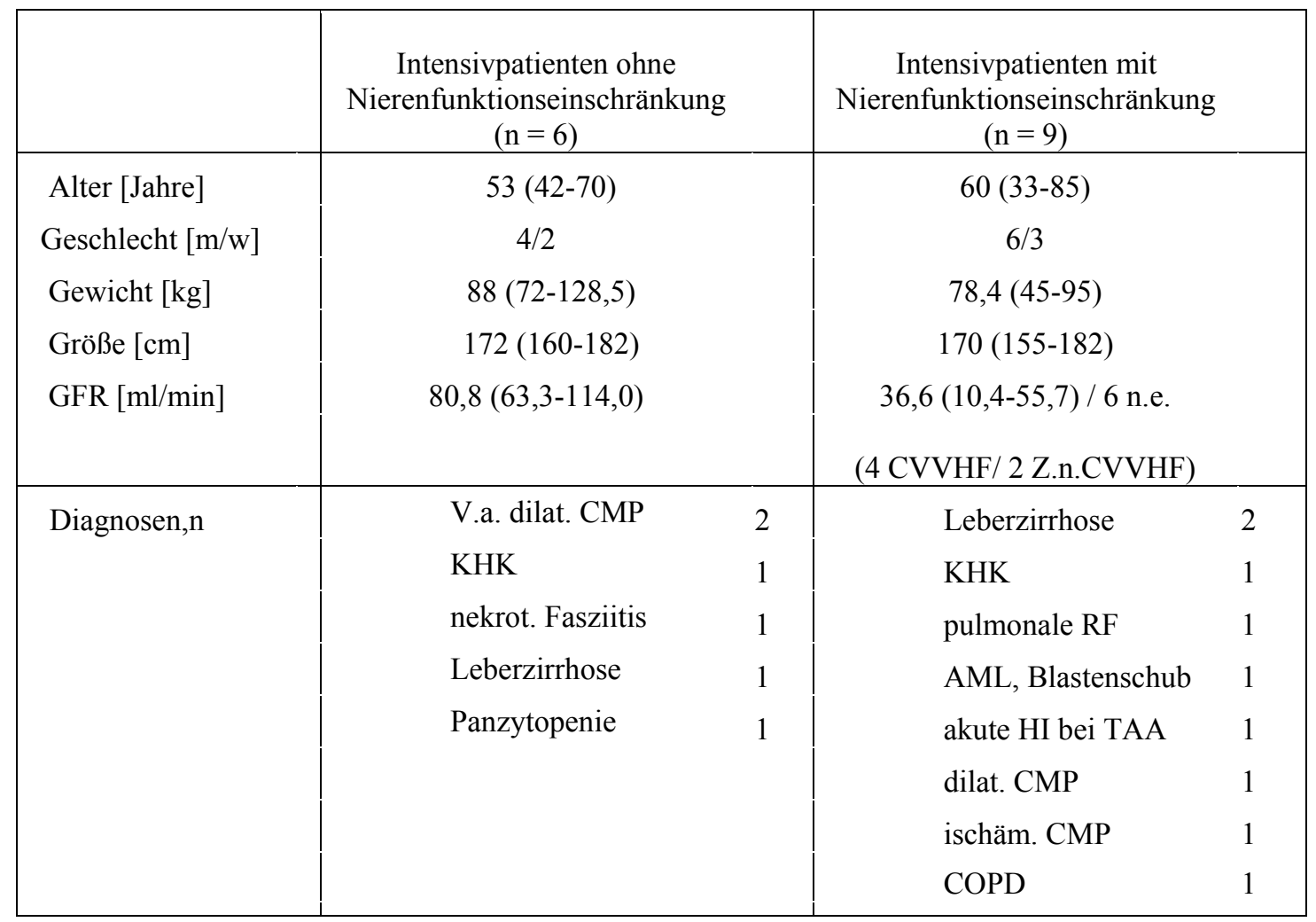

n.e. $=$ nicht ermittelt

Tabelle 5: Demographische Daten der Intensivpatienten 


\subsubsection{Laborparameter der Intensivpatienten}

Die laborchemischen Parameter (Elektrolyte, Protein, Albumin, Kreatinin, Transaminasen, Cholestasewerte), hämatologischen und hämostasiologischen Befunde sind in Tabelle 16 im Anhang zusammengefasst.

\subsubsection{Infektionsparameter der Intensivpatienten}

In der Gruppe der Intensivpatienten waren 11 Patienten, bei denen die Infektion sowohl klinisch als auch mikrobiologisch gesichert war (CMDI). Bei 4 Patienten lagen lediglich klinische Zeichen einer Infektion ohne mikrobiologischen Nachweis vor (CDI). Die Infektionsparameter der Intensivpatienten sind in den Tabellen 17 und $18 \mathrm{im}$ Anhang zusammengestellt.

\begin{tabular}{|c|c|c|}
\hline & Einschlusskriterien & Patienten Nr. \\
\hline 1) keine Sepsis: & $\begin{array}{l}\text { weniger als } 2 \text { SIRS - Kriterien (Infektion aber keine } \\
\text { Sepsis) }\end{array}$ & 14 \\
\hline $\begin{array}{l}\text { 2) wahrscheinliche } \\
\text { Sepsis: }\end{array}$ & $\begin{array}{l}\text { mindestens } 2 \text { SIRS - Kriterien und } \\
\text { a)mikrobiologischer Nachweis einer nicht } \\
\text { systemischen Infektion und/oder } \\
\text { b) neue oder persistierende Infiltrate in der Lunge }\end{array}$ & 12 \\
\hline 3) Sepsis: & $\begin{array}{l}\text { mindestens } 2 \text { SIRS - Kriterien und mikrobiologischer } \\
\text { Nachweis einer systemischen Infektion (positive Blut- } \\
\text { kultur oder positive bronchoalveoläre Lavage (BAL) }\end{array}$ & $8,11,13,23,24$ \\
\hline $\begin{array}{l}\text { 4) schwere Sepsis/ } \\
\text { septischer Schock: }\end{array}$ & $\begin{array}{l}\text { Patienten mit septischem Schock hatten über die } \\
\text { Sepsiskriterien hinaus eine Organdysfunktion (z.B. } \\
\text { Schocklunge oder akutes Nierenversagen), eine } \\
\text { Hypoperfusion mit Lactatazidose, Oligurie oder Abfall } \\
\text { des mentalen Status und eine sepsisinduzierte } \\
\text { Hypotension mit Abfall des systolischen Blutdrucks } \\
\text { um } 40 \mathrm{mmHg} \text { von der Baseline oder einen } \\
\text { Blutdruckabfall auf unter } 90 \mathrm{mmHg} \text { oder } \\
\text { Aufrechterhaltung eines Blutdrucks über } 90 \mathrm{mmHg} \\
\text { durch Einsatz positiv inotroper Substanzen oder } \\
\text { forcierter Flüssigkeitszufuhr. }\end{array}$ & $\begin{array}{l}15,16,17,19 \\
20,21,22,25\end{array}$ \\
\hline
\end{tabular}

Tabelle 5: Unterteilung der Intensivpatienten nach dem Schweregrad der Infektion 
Alle Patienten außer Patient Nr. 14 hatten eine Sepsis. Lediglich bei Patient Nr. 12 fehlten neben den Zeichen einer systemischen Reaktion die Zusatzkriterien (mikrobiologischer Nachweis einer systemischen Infektion durch positive Blutkultur oder positive bronchoalveoläre Lavage) zur Diagnose Sepsis, so dass hier eine wahrscheinliche Sepsis besteht. Unter den Sepsispatienten waren 8 Patienten mit septischem Schock. Die Schwere der Infektion ist aus Tabelle 19 im Anhang ersichtlich.

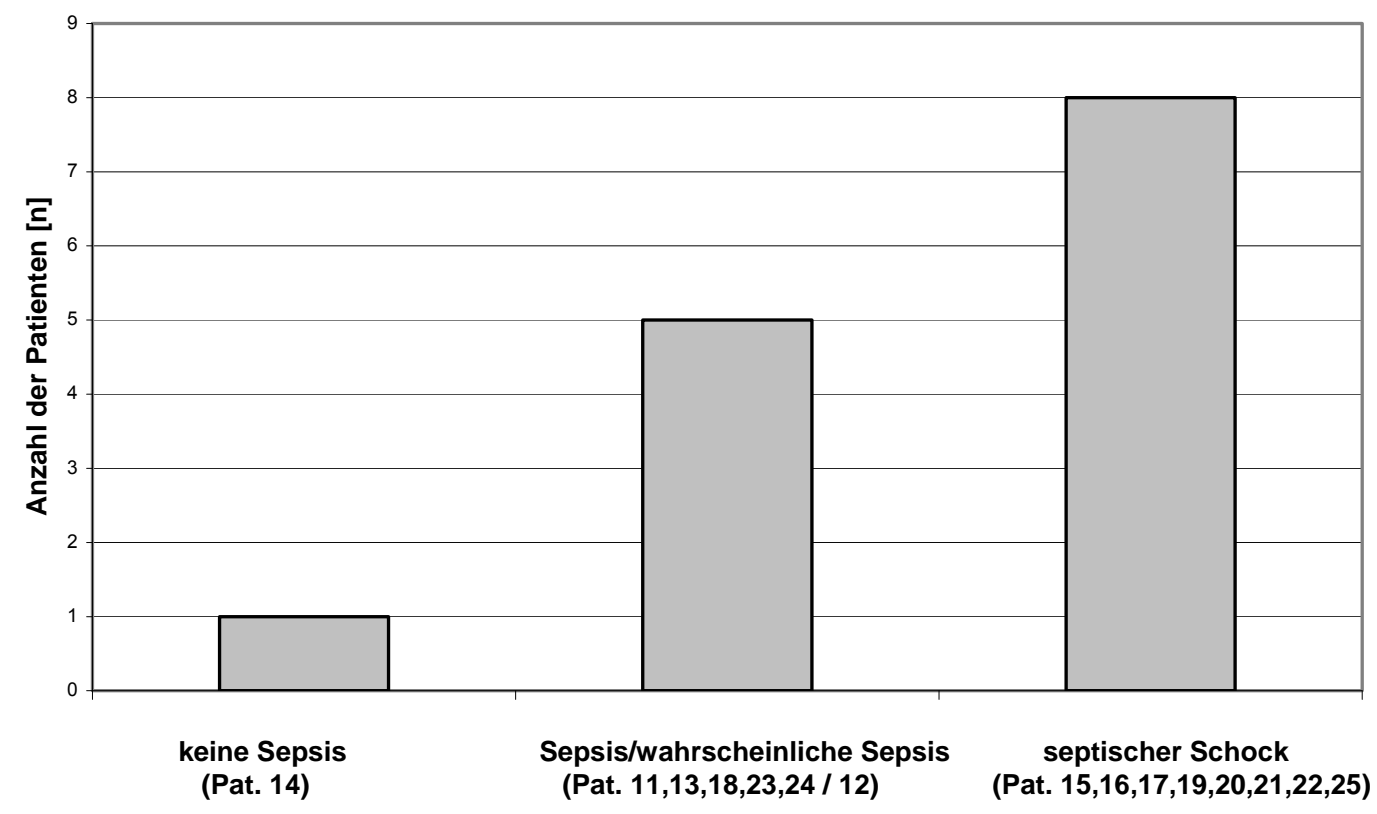

Abbildung 11: Intensivpatienten unterteilt nach Schwere der Infektion

\subsubsection{Pharmakokinetische Daten der Intensivpatienten}

7 Patienten erhielten eine Dosis von 500 mg Meropenem alle 8 Stunden, 5 Patienten erhielten $1000 \mathrm{mg}$ alle 8 Stunden, 1 Patient erhielt $500 \mathrm{mg}$ alle 12 Stunden, 2 Patienten erhielten eine Dosis von 1000 mg alle 12 Stunden. Die Dauer der Infusion betrug 24 (10 bis 79) Minuten.

Die nach Cockroft und Gault errechnete Kreatinin-Clearance [Mittelwert/Median (Range)] aller Intensivpatienten ohne CVVHF oder Zustand nach CVVHF lag bei 66,1/72,5 (10,4114,0) und war somit geringer als bei den hämatoonkologischen Patienten mit 89,1/80,2 $(55,6-126,7) \mathrm{ml} / \mathrm{min}$.

Die Meropenem-Maximumkonzentration lag bei 35,2/39,5 (9,6-70,5) mg/l, die Minimumkonzentration bei 5,2/3,3 (0,02-14,2) mg/l. Die gefundenen Maxima unterschieden sich um einen Faktor 7, die Minima um einen Faktor größer als 700. Beide Parameter unterlagen einer höheren Variabilität als bei den hämatoonkologischen Patienten. Die Maxima waren 
aufgrund der niedrigeren Dosis, der größeren Verteilungsvolumina und der zum Teil längeren Infusionszeiten kleiner als bei den hämatoonkologischen Patienten. Die Minima waren insbesondere wegen der häufig auftretenden Nierenfunktionseinschränkung und Kumulation durchschnittlich größer als bei den hämatoonkologischen Patienten $(1,1 / 0,6(<0,1-2,83)$ $\mathrm{mg} / \mathrm{l})$.

Das Verteilungsvolumen von Meropenem im Steady-State lag bei den Intensivpatienten bei 34,5/30,9 (17,5-69,4) 1 bzw. 0,43/0,40 (0,15-0,77) 1/kg und war durchschnittlich größer als bei den hämatoonkologischen Patienten mit 23,6/ 22,9 (15,0-36,8) 1 bzw. 0,33/0,33 (0,18-0,53) $1 / \mathrm{kg}$. Die Verteilungsvolumina bei den Intensivpatienten unterlagen auch einer größeren Variabilität.

Die Halbwertszeit in der Verteilungsphase $\left(\mathrm{T}_{1 / 2 \alpha}\right)$ lag bei $0,46 / 0,45(0,12-0,96)$ und entsprach den Werten der hämatoonkologischen Patienten mit 0,43/0,37 (0,21-0,74) Stunden. Die Halbwertszeit in der Eliminationsphase $\left(\mathrm{T}_{1 / 2 \beta}\right)$ lag bei 3,89/3,69 (0,80-7,21) und war größer als bei den hämatoonkologischen Patienten mit 2,03/1,69 (0,75-4,77) Stunden.

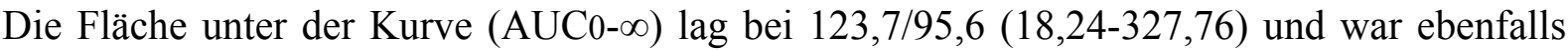
durchschnittlich größer als bei den hämatoonkologischen Patienten mit 82,77/85,4 (48,3120,3) $\mathrm{mg} / \mathrm{l} \cdot \mathrm{h}$. Es unterlag aber auch einer größeren Variabilität.

Die totale Plasmaclearance von Meropenem lag bei den Intensivpatienten bei 148,5/124 $(30,4-398,6) \mathrm{ml} / \mathrm{min}$. Die Clearance war somit durchschnittlich kleiner, aber auch variabler als bei den hämatoonkologischen Patienten mit 217.5/196.9 (138,6-345,3) ml/min.

Die MIC90 für Enterobacteriaceae (2 mg/l) wurde nach 12,43/9,72 (3,37-25,90) Stunden bzw. 89,0/100,0 (31,1-100,0) \% des Dosierungsintervalls unterschritten. Die Intensivpatienten unterschritten die Konzentration $2 \mathrm{mg} / 1$ somit durchschnittlich zu einem späteren Zeitpunkt als die hämatoonkologischen Patienten mit 6,57/6,43 (2,79-9,59) Stunden bzw. 75,1/74,3 $(34,8-100,0) \%$ des Dosierungsintervalls.

Die MIC90 für Pseudomonas aeruginosa (4 mg/l) wurde nach 8,66/7,17 (1,41-19,72) Stunden bzw. 74,4/78,9 (11,8-100) \% des Dosierungsintervalls unterschritten. Dieser Zeitraum war ebenfalls größer als bei den hämatoonkologischen Patienten mit 4,58/4,24 (2,43-7,31) Stunden bzw. 57,2/53,0 (30,4-91,4) \% des Dosierungsintervalls.

Bei den Intensivpatienten bestand kein linearer Zusammenhang $(\mathrm{R}=0,36)$ zwischen der Kreatinin- und der Meropenem-Clearance, wie es bei den hämatoonkologischen Patienten der Fall war. 


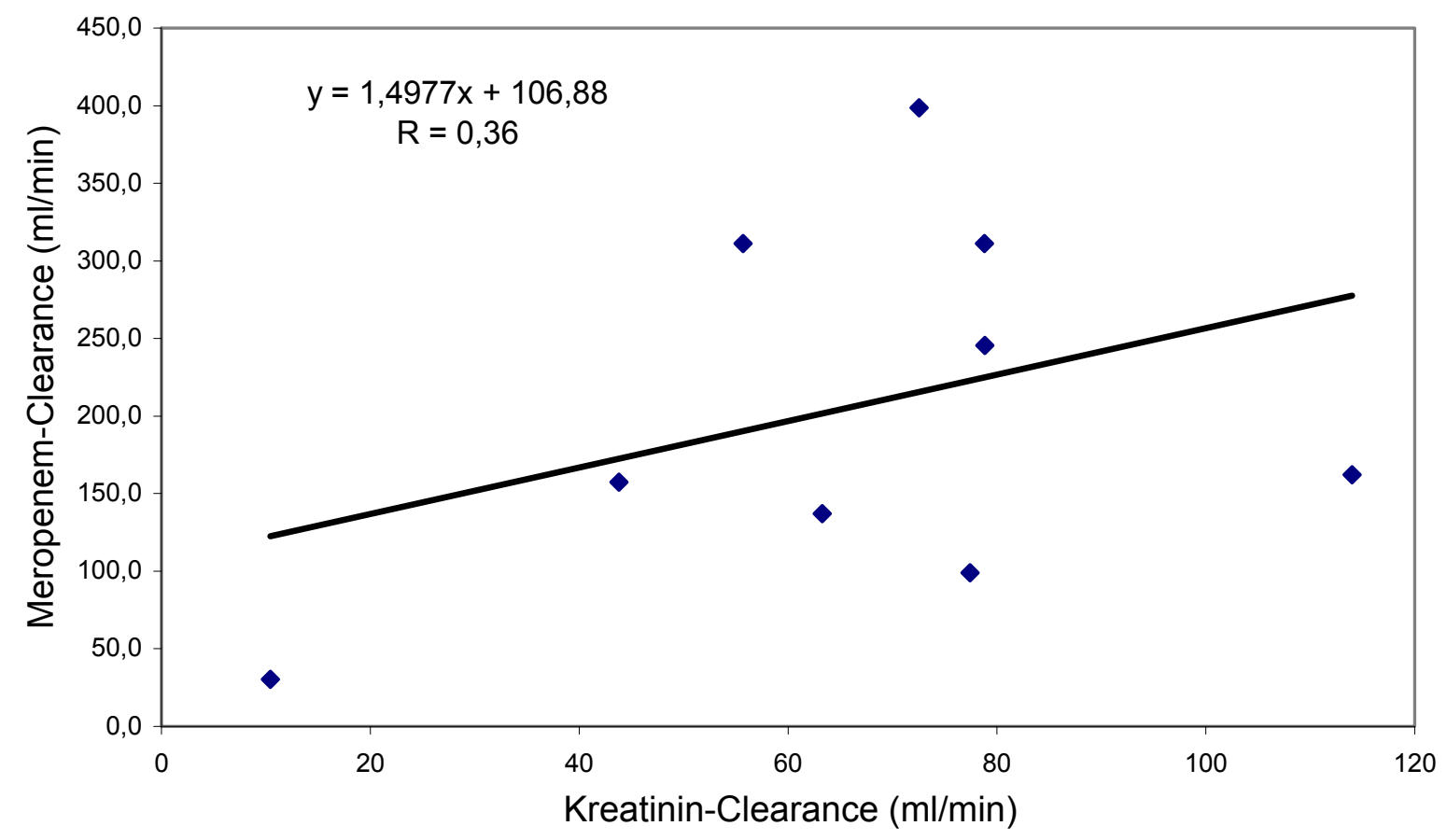

Abbildung 12: Zusammenhang zwischen Kreatinin-Clearance und Meropenem-Clearance bei Intensivpatienten ohne CVVHF oder Z.n. CVVHF

\subsubsection{Pharmakokinetische Daten der Intensivpatienten in Abhängigkeit von der Nierenfunktion}

Um den Einfluss der Nierenfunktionseinschränkung $\mathrm{zu}$ analysieren wurden die Intensivpatienten in 2 Gruppen unterteilt.

Gruppe 1: Kreatinin-Clearance $>60 \mathrm{ml} / \mathrm{min}$

Gruppe 2: Kreatinin-Clearance $<60 \mathrm{ml} / \mathrm{min}$ bzw. Notwendigkeit der Nierenersatztherapie

Die Kreatinin-Clearance wurde anhand des Serumkreatinins, der Größe, des Gewichts, des Alters und Geschlechts anhand der Formel nach Cockroft und Gault berechnet und lag bei den Intensivpatienten ohne Nierenfunktionseinschränkung (Kreatinin-Clearance $>60 \mathrm{ml} / \mathrm{min}$ ) bei $80,8 / 78,1(63,3-114,0) \mathrm{ml} / \mathrm{min}$.

Unter den Patienten mit Nierenfunktionseinschränkung wurde bei 4 Patienten zum Untersuchungszeitpunkt aufgrund eines akuten Nierenversagens eine kontinuierlich veno-venöse Hämofiltration (CVVHF) durchgeführt und 2 Patienten befanden sich im unmittelbaren Zustand nach CVVHF. Aufgrund der instabilen Nierenfunktion ließ sich bei diesen Patienten die Kreatinin-Clearance nicht berechnen. Die 3 Patienten mit Nierenfunktionseinschränkung, 
aber ohne Nierenersatztherapie, hatten eine Kreatinin-Clearance von 36,6/43,8 (10,4-55,7) $\mathrm{ml} / \mathrm{min}$.

Von den Intensivpatienten ohne Nierenfunktionseinschränkung (Kreatinin-Clearance $>60$ $\mathrm{ml} / \mathrm{min}$ ) erhielten 3 Patienten eine Dosis von $1000 \mathrm{mg}$ und 3 Patienten eine Dosis von $500 \mathrm{mg}$ alle 8 Stunden als iv.-Infusion. Die Infusionsdauer betrug 20 (13-30) Minuten.

Bei den Intensivpatienten mit Nierenfunktionseinschränkung (Kreatinin-Clearance $<60$ $\mathrm{ml} / \mathrm{min}$ oder Notwendigkeit einer Nierenersatztherapie) erhielten 2 Patienten eine Dosis von 1000 mg Meropenem alle 12 Stunden, 2 Patienten erhielten eine Dosis von $1000 \mathrm{mg}$ alle 8 Stunden, 5 Patienten erhielten eine Dosis von $500 \mathrm{mg}$ alle 8 Stunden als iv.-Infusion. Die Infusionsdauer betrug 26 (10-79) Minuten.

Bei den Intensivpatienten ohne Nierenfunktionseinschränkung lag die Meropenem-Maximumkonzentration am Ende der Infusion bei 32,4/33,4 (13,5-45,3) mg/1. Die Maxima unterschieden sich um den Faktor 3,4.

Die Meropenem-Maximumkonzentration bei den Intensivpatienten mit Nierenfunktionseinschränkung lag bei 35,7/30,6 (9,6-70,5) mg/l. Die Maxima unterschieden sich hier um einen Faktor 7,3. Die Maximumkonzentrationen waren somit vergleichbar mit den Werten bei Patienten ohne Nierenfunktionseinschränkung.

Die Meropenem-Minimumkonzentration bei den Patienten ohne Nierenfunktionseinschränkung lag bei 1,8/0,8 $(<0,1-5,8) \mathrm{mg} / \mathrm{l}$. Die Minima unterschieden sich somit um einen Faktor größer als 58.

Bei den Patienten mit Nierenfunktionseinschränkung lagen die Minimumkonzentrationen bei 6,87/4,36 (2,3-14,2) mg/l. Sie unterschieden sich um den Faktor 6,2 und waren durchschnittlich 3,8-mal größer als bei den Patienten ohne Nierenfunktionseinschränkung. Es bestand ein signifikanter Unterschied zwischen beiden Gruppen $(\mathrm{p}=0,035)$.

Die Plasmakonzentrationsverläufe in Abhängigkeit von der Dosis und der Nierenfunktionseinschränkung sind in der folgenden Abbildung dargestellt (Abbildung 13). 


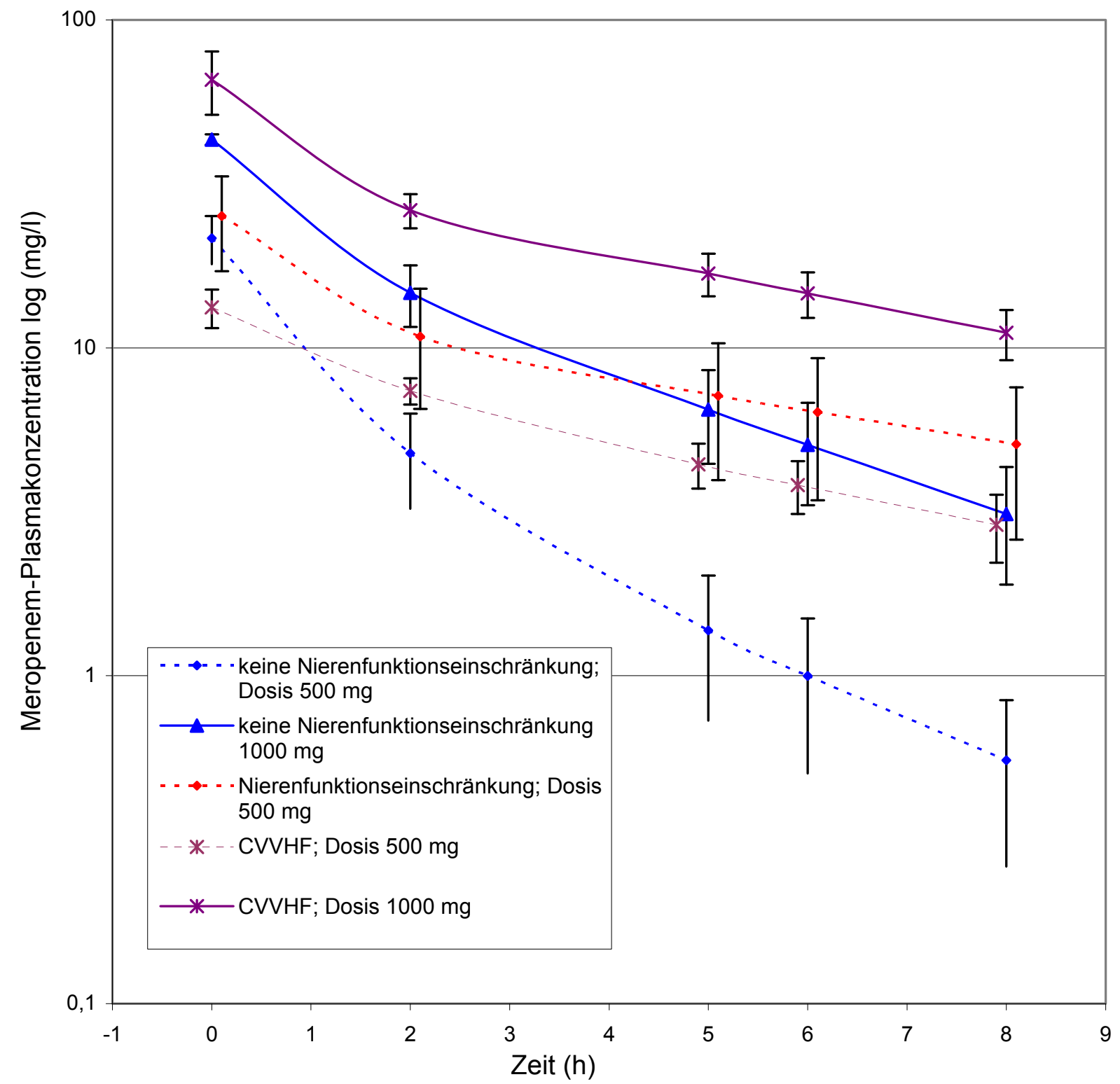

Abbildung 13: Plasmakonzentrationsverläufe der Intensivpatienten in Abhängigkeit von der Dosis und dem Ausmaß der Nierenfunktionseinschränkung

Das Verteilungsvolumen im Steady-State $\left(\mathrm{V}_{\mathrm{ss}}\right)$ lag bei den Intensivpatienten ohne Nierenfunktionseinschränkung bei 33,6/27,0 (19,5-69,4) 1 bzw. 0,40/0,36 $(0,15-0,77) 1 / \mathrm{kg}$ und war somit etwa so groß wie bei den Patienten mit Nierenfunktionseinschränkung. Hier betrug das Verteilungsvolumen 35,1/32,0 (17,5-52,2) 1 bzw. 0,45/0,48 (0,22-0,58) 1/kg) und hatte eine ähnlich große Variabilität. Das Ausmaß der Nierenfunktionseinschränkung hatte somit keinen Einfluss auf das Verteilungsvolumen.

Die Halbwertszeit in der Verteilungsphase $\left(\mathrm{T}_{1 / 2 \alpha}\right)$ betrug bei den Intensivpatienten ohne Nierenfunktionseinschränkung 0,42/0,44 $(0,12-0,69)$ Stunden und bei den Intensivpatienten 
mit Nierenfunktionseinschränkung 0,49/0,46 (0,17-0,96) Stunden. Die Halbwertszeiten in der Verteilungsphase unterlagen mit $\mathrm{p}=0,596$ keinem signifikanten Unterschied.

Die Halbwertszeit in der Eliminationsphase $\left(T_{1 / 2 \beta}\right)$ lag bei den Patienten ohne Nierenfunktionseinschränkung bei 2,24/2,44 (0,80-3,19) Stunden. Bei den Patienten mit Nierenfunktionseinschränkung betrug die Halbwertszeit in der Eliminationsphase 4,99/5,27 (3,28$7,21)$ Stunden und war mit $\mathrm{p}=0,007$ signifikant größer als bei den Patienten ohne Nierenfunktionseinschränkung.

Die Fläche unter der Zeit-Konzentrations-Kurve $\left(\mathrm{AUC}_{0-\infty}\right)$ betrug bei den Patienten ohne Nierenfunktionseinschränkung 68,5/57,4 (18,2-156,8) $\mathrm{mg} \cdot \mathrm{h} / \mathrm{l}$ und bei den Patienten mit Nierenfunktionseinschränkung 160,5/137,6 $(48,9-327,8) \mathrm{mg} \cdot \mathrm{h} / \mathrm{l}$. Es bestand ein deutlicher Unterschied zwischen beiden Gruppen, allerdings ohne statistische Signifikanz ( $p=0,075)$.

Die totale Plasmaclearance von Meropenem lag bei den Intensivpatienten ohne Nierenfunktionseinschränkung bei 225,6/203,9 (99,1-398,6) $\mathrm{ml} / \mathrm{min}$. Bei den Patienten mit Nierenfunktionseinschränkung lag die Plasmaclearance bei 97,0/98,4 (30,4-157,3) ml/min. Sie war somit bei den Patienten ohne Nierenfunktionseinschränkung signifikant größer als bei den Patienten mit Nierenfunktionseinschränkung $(\mathrm{p}=0,0087)$.

Die Meropenem-Clearance der Patienten mit CVVHF und Z.n. CVVHF lag mit 93,6 (50,9 147,6) $\mathrm{ml} / \mathrm{min}$ in der Größenordnung wie bei Patienten mit einer glomerulären Filtrationsrate von $30-60 \mathrm{ml} / \mathrm{min}$.

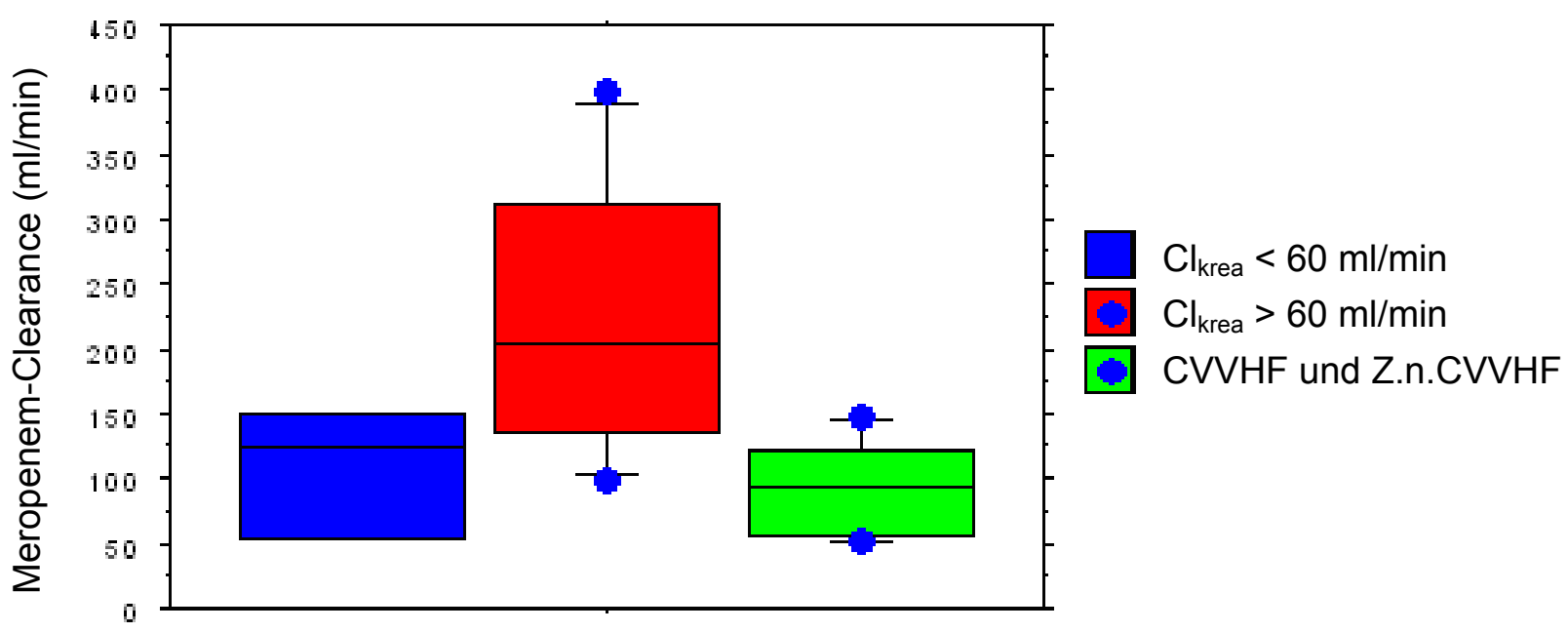

Abbildung 14: Meropenem-Clearance der Intensivpatienten unterteilt nach dem Ausmaß der Nierenfunktionseinschränkung 
Die MIC90 von Enterobacteriaceae (2 mg/l) wurde von den Patienten mit intakter Nierenfunktion nach 8,19/6,70 (3,37-18,91) Stunden bzw. 73,5/83,8 (31,1-100,0) \% des Dosierungsintervalls unterschritten. Bei den Patienten mit eingeschränkter Nierenfunktion wurden die entsprechende Konzentration nach 15,3/14,1 (8,29-25,9) Stunden bzw. 98,4/100,0 (94,9100,0) \% des Dosierungsintervalls unterschritten. Der Unterschied war mit $p=0,051$ auch hier deutlich, wenn auch nicht signifikant.

Die MIC90 von Pseudomonas aeruginosa (4 mg/l) wurde von den nierengesunden Patienten nach 5,49/4,30 (1,41-13,35) Stunden bzw. 56,5/53,7 (11,8-100,0) \% des Dosierungsintervalls und von den Patienten mit eingeschränkter Nierenfunktion nach 10,7/8,9 (4,8-19,7) Stunden bzw. 86,4/100,0 (60,0-100,0) \% des Dosierungsintervalls unterschritten. Auch hier bestanden zwischen beiden Gruppen mit $\mathrm{p}=0,069$ deutliche Unterschiede.

\subsubsection{Pharmakokinetische Daten der Intensivpatienten in Abhängigkeit von der Schwere der Infektion}

Unterteilt man die Intensivpatienten nach der Schwere ihrer Infektion in Patienten ohne Sepsis $(n=1)$, Patienten mit Sepsis ohne septischen Schock $(n=6)$ und Patienten mit Sepsis und septischem Schock $(n=8)$ zeigen sich ebenfalls Unterschiede beim Vergleich der pharmakokinetischen Parameter unabhängig von der Nierenfunktionseinschränkung.

Die Meropenem-Minimumkonzentration bei dem Patienten ohne Sepsis $(n=1)$ lag bei 2,9 mg/l. Bei den Patienten mit Sepsis $(n=6)$ lagen die Minimumkonzentration bei 3,9/1,7 $(0,02-$ 14,2) mg/l. Bei den Patienten mit septischem Schock $(n=8)$ lag die Minimumkonzentrationen bei $6,6 / 1,7(2,3-13,7) \mathrm{mg} / \mathrm{l}$.

Das Verteilungsvolumen im Steady-State $\left(\mathrm{V}_{\mathrm{ss}}\right)$ lag bei dem Patienten ohne Sepsis bei 36,1 1 bzw. 0,49 1/kg. Bei den Patienten mit Sepsis bei 29,3/25,6 (19,5-52,2) 1 bzw. 0,38/0,40 (0,150,57) $1 / \mathrm{kg}$ und bei den Patienten mit septischem Schock bei 38,3/38,1 (17,5-69,4) 1 bzw. $0,46 / 0,45(0,22-0,77) 1 / \mathrm{kg}$.

Die Intensivpatienten mit septischem Schock tendierten zu höheren Verteilungsvolumina als Patienten ohne septischen Schock unabhängig vom Ausmaß der Niereninsuffizienz. 


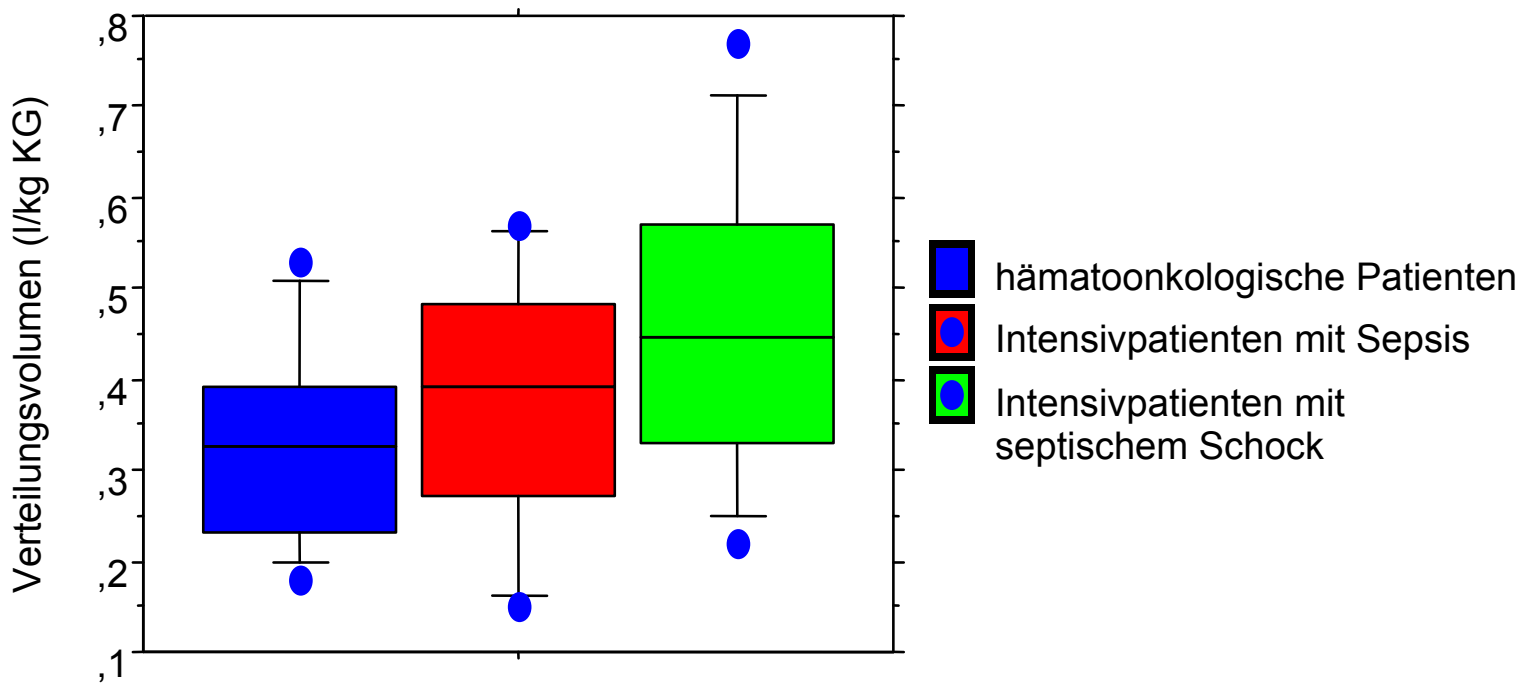

Abbildung 15: Verteilungsvolumen bei hämatoonkologischen Patienten, Intensivpatienten mit Sepsis und Intensivpatienten mit septischem Schock

Die Patienten mit septischem Schock tendierten zu einer schlechteren Nierenfunktion als Patienten ohne septischen Schock (Abbildung 16).

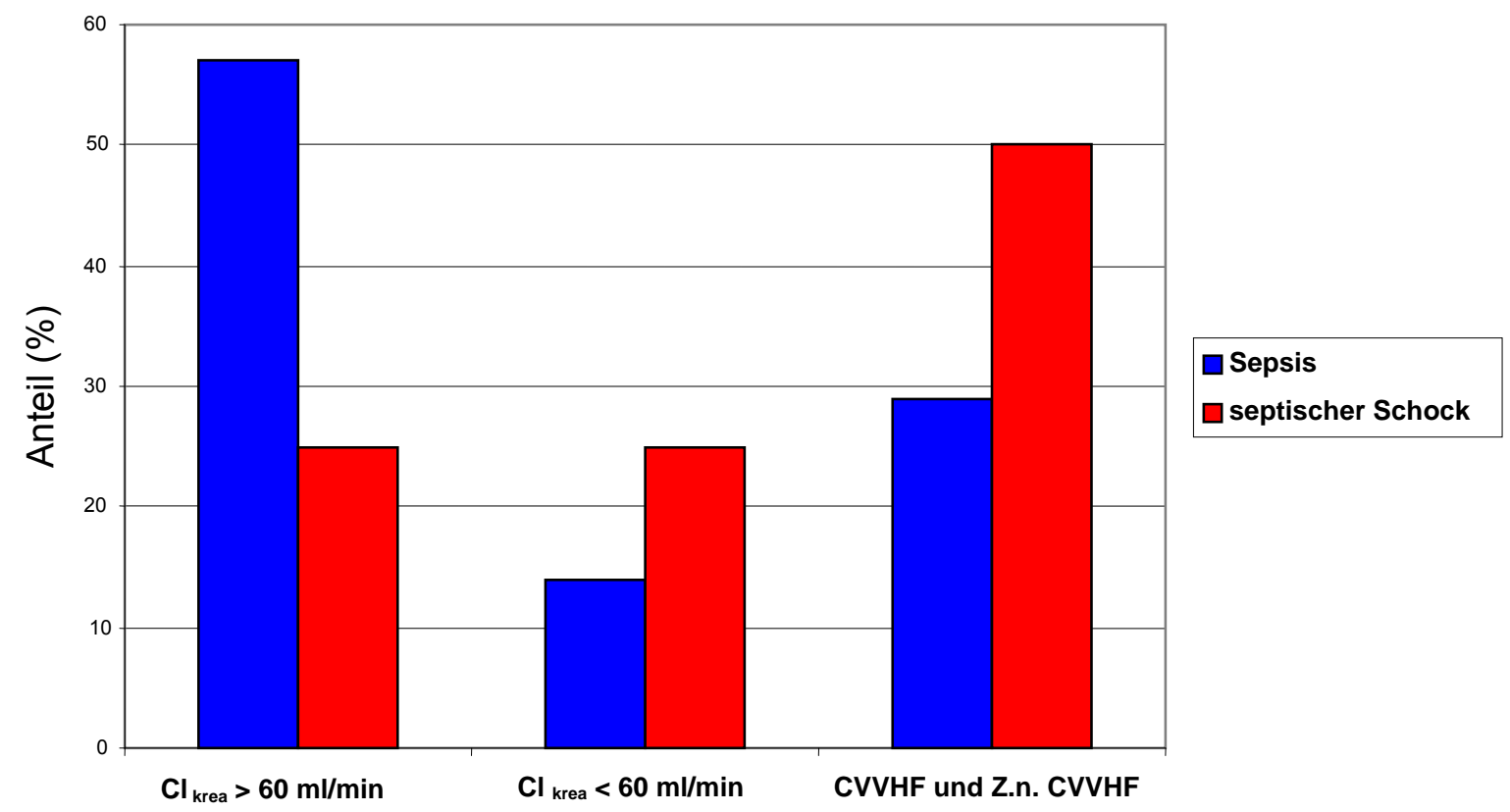

Abbildung 16: Anteil der Patienten mit und ohne Nierenfunktionseinschränkung unter den Patienten mit Sepsis und septischem Schock

Unter den Intensivpatienten mit Sepsis ohne septischen Schock waren 4 Patienten mit einer Kreatinin-Clearance $>60 \mathrm{ml} / \mathrm{min}, 1$ Patienten mit einer Kreatinin-Clearance von $<60 \mathrm{ml} / \mathrm{min}$ und 2 Patienten bei denen wegen eines akuten Nierenversagens eine kontinuierliche veno- 
venöse Hämofiltration zum Einsatz kam. Unter den Patienten mit septischem Schock waren je 2 Patienten mit einer Kreatinin-Clearance größer und kleiner als 60 ml/min, 2 Patienten mit akutem Nierenversagen und CVVHF und 2 Patienten mit Z.n. CVVHF bei akutem Nierenversagen.

Bei Intensivpatienten mit septischem Schock war auch die Meropenem-Clearance häufig erniedrigt. Sie lag bei den Patienten mit septischem Schock bei 130,2/98,8 (30,4-398,6) $\mathrm{ml} / \mathrm{min}$ und Patienten ohne septischen Schock bei 170,5/147,2 (50,9-311,3) ml/min. Aufgrund der großen Variabilität bestand zwischen beiden Gruppen jedoch kein signifikanter Unterschied.

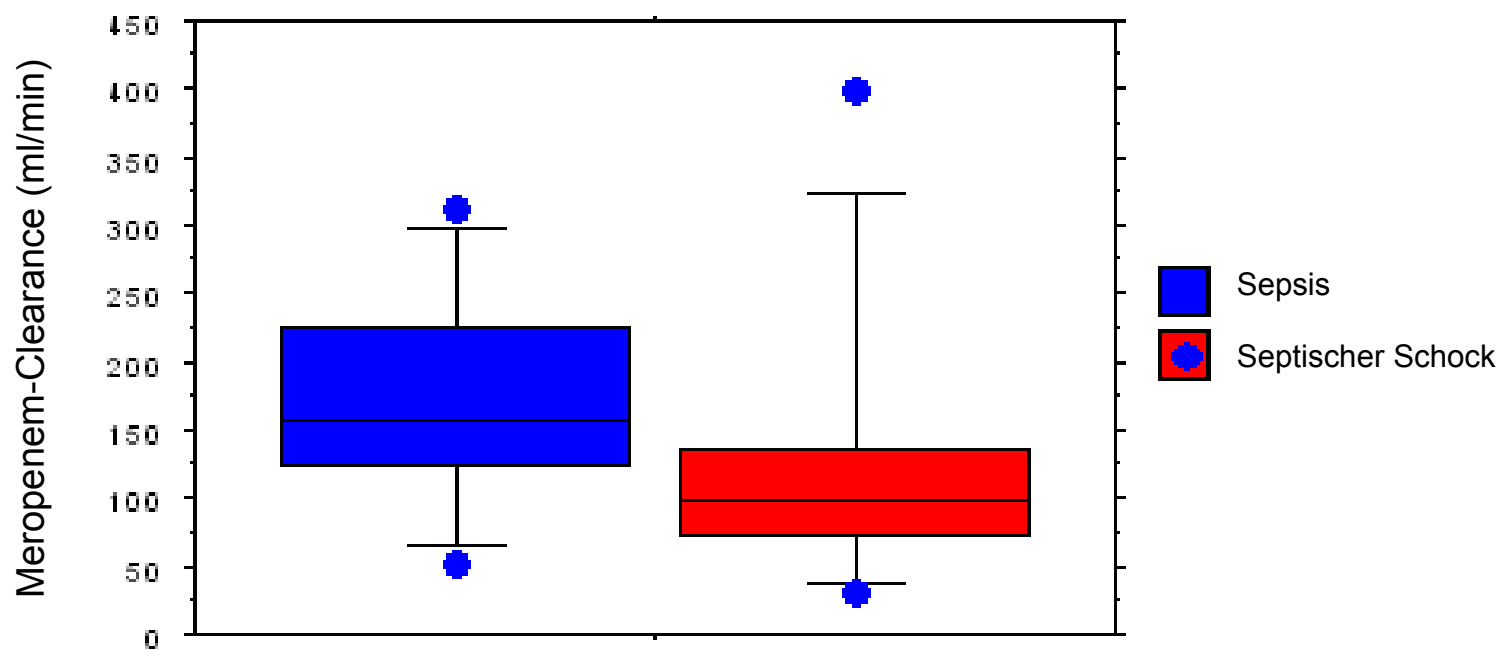

Abbildung 17: Meropenem-Clearance der Intensivpatienten unterteilt nach Sepsis und septischem Schock

Die Halbwertszeit in der Verteilungsphase $\left(T_{1 / 2 \alpha}\right)$ betrug bei dem Intensivpatienten ohne Sepsis 0,43 Stunden. Bei den Intensivpatienten mit Sepsis ohne septischen Schock 0,36/0,35 (0,17$0,69)$ Stunden und bei den Intensivpatienten mit septischem Schock 0,54/0,53 (0,12-0,96) Stunden. Der Unterschied zwischen den beiden letztgenannten Gruppen war mit $\mathrm{p}=0,19$ deutlich, wenn auch nicht signifikant.

Die Halbwertszeit in der Eliminationsphase $\left(T_{1 / 2 \beta}\right)$ lag bei den Intensivpatienten mit Sepsis ohne septischen Schock bei 2,91/2,90 (0,8-5,27) Stunden. Bei den Patienten mit septischem Schock betrug die terminale Halbwertszeit 4,74/4,72 (2,37-7,21) und war mit $\mathrm{p}=0,062$ deutlich größer als bei den Patienten ohne septischen Schock. 
Die Fläche unter der Zeit-Konzentrations-Kurve (AUC0- $\infty$ ) betrug bei den Patienten mit Sepsis ohne septischen Schock 109,0/57,4 (24,0-327,8) $\mathrm{mg} \cdot \mathrm{h} / \mathrm{l}$ und bei den Patienten mit septischem Schock 138,0/126,0 (18,2-290,4) mg.h/l. Es bestand in beiden Gruppen eine hohe Variabilität. Ein Unterschied zwischen beiden Gruppen war nicht erkennbar.

Die MRT (mean residence time) als Quotient aus Verteilungsvolumen und Clearance betrug bei den Intensivpatienten mit Sepsis ohne septischen Schock 226,6/210,0 (62,7-428,2) min und bei den Intensivpatienten mit septischem Schock 371,5/356,8 (174,2-575,8) min. Es bestand mit $\mathrm{p}=0,071$ ein deutlicher Unterschied.

Die minimale Hemmkonzentration von Enterobacteriaceae (2 mg/l) wurde von den Patienten mit Sepsis ohne septischen Schock nach 10,60/8,15 (3,37-25,90) Stunden bzw. 84,1/89,8 $(42,1-100,0) \%$ des Dosierungsintervalls unterschritten. Bei den Patienten mit septischem Schock wurden die entsprechenden Konzentrationen nach 14,14/15,13 (3,73-23,06) Stunden bzw. 91,4/100 (31,1-100,0) \% des Dosierungsintervalls unterschritten. Es bestand kein wesentlicher Unterschied zwischen beiden Gruppen.

Die Plasmakonzentration von $4 \mathrm{mg} / \mathrm{l}$ als minimale Hemmkonzentration von Pseudomonas aeruginosa wurde von den Intensivpatienten ohne septischen Schock 7,43/5,06 (2,40-19,72) Stunden bzw. 62,6/63,2 (30,0-100,0) \% des Dosierungsintervalls und von den Patienten mit septischem Schock nach 9,76/9,70 (1,41-17,26) Stunden bzw. 81,3/100,0 (11,75-100,0) \% des Dosierungsintervalls unterschritten. Auch hier bestand zwischen beiden Gruppen mit $p=0,49$ kein wesentlicher Unterschied.

\subsubsection{Outcome der Intensivpatienten}

In der Gruppe der Intensivpatienten hatten 3 Patienten einen guten Outcome mit Entfieberung nach weniger als 3 Tagen (Patient 12, 13, 19). 3 Patienten hatten einen befriedigenden Outcome mit Entfieberung unter Meropenem nach mehr als 3 Tagen (Patient 11, 15, 24). 5 Patienten hatten einen mangelhaften Outcome ohne Entfieberung trotz Therapie mit Meropenem über mehr als 3 Tage (Patient 14, 17, 18, 21, 22) und 4 Patienten einen unbefriedigenden Outcome mit Tod anderer Ursache als Infektion (Patient 16, 20, 23, 25). 


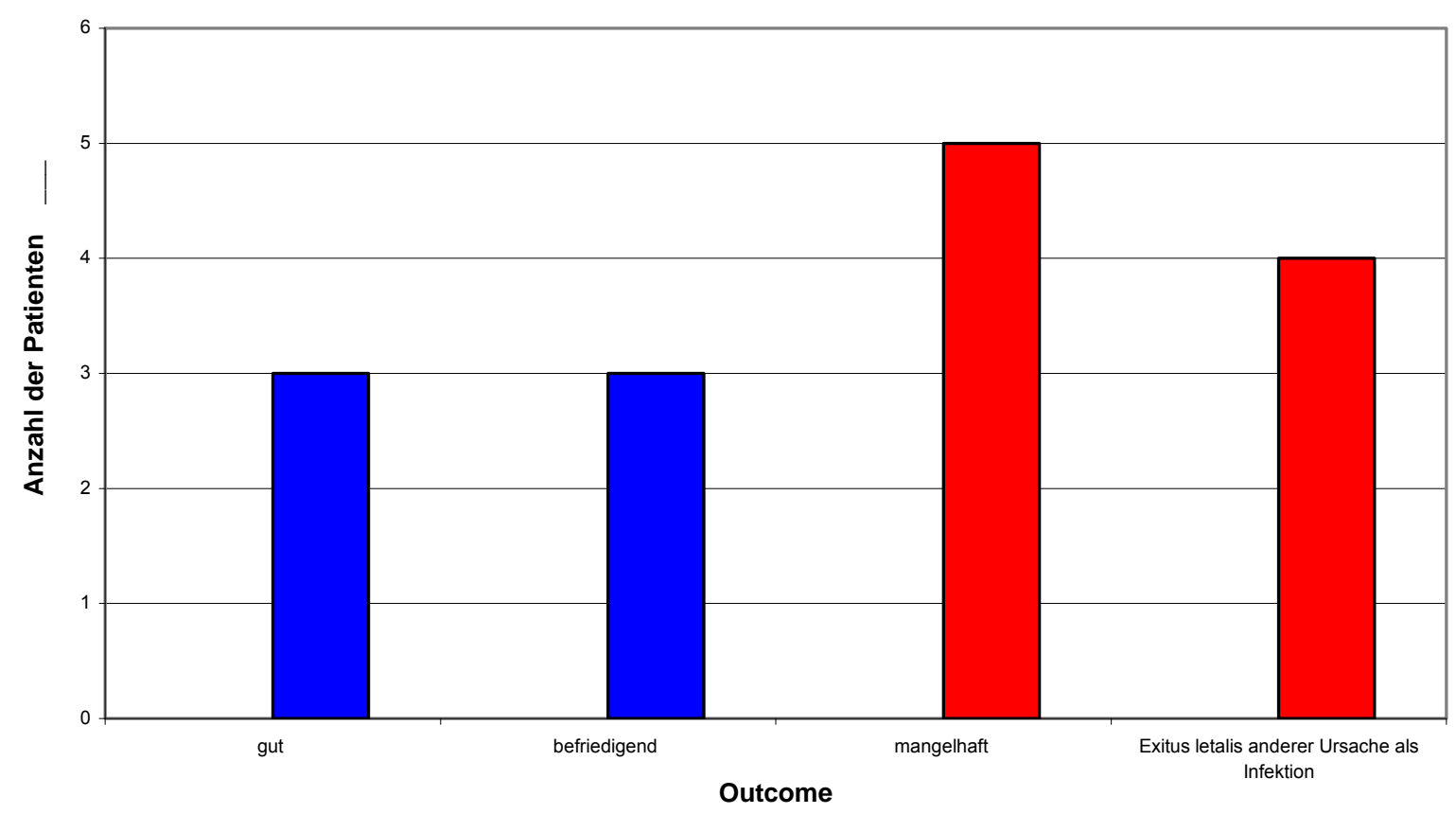

Abbildung 18: Outcome der Intensivpatienten

Bei den Patienten mit gutem bzw. befriedigendem Outcome handelt es sich in 3 Fällen um Infektionen mit sensiblen Keimen. In 3 Fällen konnte kein Erregernachweis geführt werden, allerdings haben diese Patienten gute bis sehr gute Meropenem-Plasmaspiegel, die die MIC des vermuteten zu bekämpfenden Keims über mindestens $65 \%$ des Dosierungsintervalls überschritten.

Bei den Patienten mit mangelhaftem Outcome bestehen bei 2 Patienten sehr schlechte Spiegel mit früher Unterschreitung der MIC relevanter Keime (Patient 17 und 18). Und bei 3 Patienten (Patient 14, 21 und 22) lagen resistente Keime vor, so dass es trotz relativ guter Spiegel zu keiner Entfieberung unter Meropenem kam.

4 Patienten verstarben an einer anderen Ursache als Infektion, so dass keine Aussagen bezüglich der Effektivität der Meropenem-Therapie getroffen werden können. 


\section{Diskussion}

Das Carbapenem-Antibiotikum Meropenem ist seit vielen Jahren erfolgreich im klinischen Gebrauch. Aufgrund seiner hohen Wirkintensität und seines breiten Wirkspektrums wird es insbesondere bei der Behandlung schwerer Infektionen mit oft unbekannten Erregern eingesetzt (Schuster und Werdan 2000).

In einer Vielzahl von Studien wurden die pharmakokinetischen und pharmakodynamischen Eigenschaften dieses Breitspektrumantibiotikums bereits analysiert und „Standarddosierungen“ etabliert (Bax et al. 1989, Brismar et al. 1995, Chimata et al. 1993, Christensson et al. 1992, Dreetz et al. 1996, Drusano et al. 2000, Hurst und Lamb 2000, Kelly et al. 1995, Leroy et al. 1992, Mouton und van den Anker 1995, Sieger 1998). Die Ergebnisse dieser Studien basieren jedoch überwiegend auf Untersuchungen gesunder Freiwilliger und von Patienten mit leichten Infektionen. Hier konnte eine gute Dosis-Konzentrations-Wirkungskorrelation gezeigt werden (Brismar et al. 1995, Kelly et al. 1995, Drusano et al. 2000). Meropenem wird jedoch insbesondere bei Patienten mit schweren Infektionen und Sepsis angewandt (Schuster und Werdan 2000). Obwohl die pharmakokinetischen und pharmakodynamischen Zusammenhänge bei diesen kritisch kranken Patienten nur unzureichend geklärt sind, erfolgt auch hier häufig die Anwendung von „Standarddosierungen“.

In der vorliegenden Studie konnte gezeigt werden, dass es unter Anwendung von Meropenem- „Standarddosierungen“ bei kritisch kranken Patienten mit schweren Infektionen in signifikanter Zahl zum Therapieversagen mit erhöhter Morbidität und Mortalität kommt. Hierfür wurden die pharmakodynamischen und pharmakokinetischen Kenngrößen von Meropenem bei 10 ausgewählten febrilen hämatoonkologischen Patienten sowie 15 nichthämatoonkologischen internistischen Patienten auf Intensivstation mit schweren Infektionen und Sepsis erhoben und analysiert. Als zugrunde liegender potentieller Mechanismus für das häufige Therapieversagen wurde die frühzeitige Unterschreitung effektiver Meropenem-Wirkspiegel infolge pathophysiologischer Veränderungen im Rahmen der Infektion und ihrer begleitenden systemischen Reaktion gesehen. Die Ursachen der veränderten Dosis-Konzentrations-Wirkungsbeziehung von Meropenem bei kritisch kranken Patienten wurden evaluiert und therapeutische Konsequenzen erarbeitet. 


\subsection{Therapieversagen unter Meropenem-Therapie}

In der vorliegenden Arbeit konnte gezeigt werden, dass es im Rahmen der Infektbehandlung unter Anwendung von Meropenem-,,Standarddosierungen“ trotz nachgewiesener Empfindlichkeit der Keime sowohl bei febrilen hämatoonkologischen Patienten, als auch bei Intensivpatienten mit schweren Infektionen in signifikanter Zahl zum Therapieversagen kommen kann.

Bei insgesamt 10 der 25 von uns untersuchten kritisch kranken Patienten (40\%) kam es zum Therapieversagen. In 6 Fällen (24 \%) kam es zur Persistenz der Infektion trotz MeropenemTherapie nach Normogramm über mehr als 3 Tage. Bei diesen Patienten konnte keine klinische Heilung der jeweils vorliegenden Infektion erreicht werden. Weitere 4 Patienten $(16 \%)$ verstarben an anderen Ursachen als Infektion.

Unter den 25 von uns untersuchten Patienten waren 10 febrile hämatoonkologische Patienten und 15 nicht-hämatoonkologische Intensivpatienten mit schweren Infektionen, Sepsis und septischem Schock.

Unter den 10 febrilen hämatoonkologischen Patienten waren 6 neutropenische (neutrophile Granulozyten $<1000 / \mu \mathrm{l})$ und 4 nicht-neutropenische Patienten. Bei 2 Patienten $(20 \%)$ kam es $\mathrm{zu}$ einem Therapieversagen der Antibiotikatherapie mit Meropenem. Bei einem dieser Patienten konnte keine Entfieberung unter Meropenem erreicht werden und bei einem weiteren Patienten kam es zum Tod infolge Infektion. Beide Patienten hatten eine Neutropenie.

Auch andere Autoren beobachteten einen hohen Anteil von Therapieversagern unter Meropenem-Therapie bei neutropenischen Patienten mit Fieber. Feld et al. fanden in dieser Patientengruppe unter Anwendung von 3-mal täglich 1000 mg Meropenem einen Anteil an klinischen Non-Respondern von 46\% (Feld et al. 2000). Behre et al. fanden einen ähnlichen hohen Anteil an Therapieversagern bei Patienten mit hämatoonkologischen oder soliden Tumoren sowie Fieber in Neutropenie von 41\% am Ende der unmodifizierten Therapie mit 3mal täglich 1000 mg Meropenem (Behre et al. 1998).

Unter den 15 von uns untersuchten Intensivpatienten mit schweren Infektionen, Sepsis und septischem Schock kam es bei 8 Patienten (53\%) zu einem Therapieversagen. Bei 4 Patienten (27\%) konnte keine Entfieberung unter Meropenem erreicht werden und bei 4 weiteren Patienten kam es zum Tod anderer Ursache als Infektion.

Ähnliche Ergebnisse bei kritisch kranken Patienten unter Meropenem-Therapie wurden auch in früheren Studien gesehen. Nach Colardyn und Faulkner hatten Patienten mit schweren bakteriellen Infektionen, die mit 3-mal täglich 1000 mg Meropenem behandelt wurden, einen 
klinischen Response von durchschnittlich 76\%. Der Erfolg der antibiotischen Therapie war allerdings direkt von der Erkrankungsschwere abhängig. Bei leichter erkrankten Patienten (APACHE II Score < 10) kam es mit 7\% klinischen Non-Respondern deutlich seltener zu einem Therapieversagen als bei kritisch kranken Patienten (APACHE II-Score $>20$ ), bei denen es unter Meropenem-,,Standarddosierung“ in 40\% zum Therapieversagen kam (Colardyn und Faulkner 1996).

\subsection{Unterschreitung erforderlicher Wirkspiegel von Meropenem}

Als potentielle Ursache für das Versagen der Antibiotikatherapie mit Meropenem bei kritisch kranken Patienten wurde häufig eine frühzeitige Unterschreitung erforderlicher Wirkspiegel gesehen. Wie bei anderen Beta-Lactam-Antibiotika besteht bei Meropenem eine zeitabhängige bakterizide Aktivität sowohl gegen grampositive als auch gegen gramnegative Keime. Für eine effektive antibakterielle Wirksamkeit und erfolgreiche Therapie ist eine Überschreitung der Hemmkonzentration des zu bekämpfenden Keims über mindestens 6070\% des Dosierungsintervalls erforderlich (Cars 1997, Craig 1998, Turnidge 1998). Bei immuninkompetenten Patienten wird sogar eine Überschreitung der minimalen Hemmkonzentrationen während des gesamten Dosierungsintervalls empfohlen (Pea und Viale 2006). Diese Mindestvoraussetzungen für eine effektive antibiotische Therapie wurden von vielen Patienten nicht erreicht.

Unter der empfohlenen Standarddosierung von 3-mal täglich 1000 mg Meropenem wurde bei den von uns untersuchten febrilen hämatoonkologischen Patienten die erforderliche Wirkkonzentration zur Bekämpfung der eigentlich hochsensiblen Enterobacteriaceae (MIC90 = 2 mg/l) nach durchschnittlich 75\% (35 - 100\%) des Dosierungsintervalls unterschritten. Bei den intermediären Keimen war die Situation noch schlechter. Die MIC90 für Pseudomonas aeruginosa (4 mg/l) wurde sogar schon nach 57\% (27 - 89\%) des Dosierungsintervalls unterschritten. Bei keinem der von uns untersuchten hämatoonkologischen Patienten konnten ausreichend hohe Hemmkonzentrationen zur Bekämpfung sensibler (2 mg/l) und intermediärer Keime (4 mg/l) während des gesamten Dosierungsintervalls aufrechterhalten werden.

Auf hämatoonkologischen und Intensivstationen liegen bekanntermaßen vermehrt Antibiotikaresistenzen vor. Bei weniger sensiblen Pseudomonaden (MIC90 $=8 \mathrm{mg} / \mathrm{l}$ ) und Acinetobacter species (MIC90 = 16 mg/l) sind die erforderlichen Konzentrationen unter der angegebenen Dosierung kaum noch zu erreichen. 
Bei den Intensivpatienten wurden unter Standarddosierung mit 2- bis 3-mal täglich 500 oder $1000 \mathrm{mg}$ Meropenem die Hemmkonzentrationen für sensible Keime $(2 \mathrm{mg} / \mathrm{l})$ nach durchschnittlich 89\% (31-100\%) und für intermediäre Keime (4 mg/l) nach durchschnittlich 74\% (11-100\%) des Dosierungsintervalls unterschritten. In Abhängigkeit von der Nierenfunktion gab es zwischen den einzelnen Patienten große Unterschiede.

Bei den Intensivpatienten mit normaler Nierenfunktion $\left(\mathrm{Cl}_{\text {krea }}>60 \mathrm{ml} / \mathrm{min}, \mathrm{n}=6\right)$ wurde die MIC90 für Enterobacteriaceae (2 mg/l) nach 73\% (31-100\%) und die MIC90 für Pseudomonas aeruginosa (4 mg/l) bereits nach 56\% (11-100\%) des Dosierungsintervalls unterschritten.

Die Intensivpatienten mit Nierenfunktionseinschränkung $\left(\mathrm{Cl}_{\text {krea }}<60 \mathrm{ml} / \mathrm{min}, \mathrm{n}=9\right)$ unterschritten die MIC90 für Enterobacteriaceae (2 mg/l) nach durchschnittlich 99\% (94-100\%) und die MIC90 für Pseudomonas aeruginosa (4 mg/l) nach durchschnittlich 86\% (60-100\%) des Dosierungsintervalls.

Nur 6 der 15 Intensivpatienten überschritten die erforderlichen Hemmkonzentrationen für sensible und intermediäre Keime während des gesamten Dosierungsintervalls. Bei all diesen Patienten lag eine Nierenfunktionseinschränkung vor, wie sie häufig im Rahmen schwerer Infektionen mit Organversagen auftreten kann.

\subsection{Ursachen der Unterschreitung erforderlicher Wirkspiegel von Meropenem}

\subsubsection{Hämatoonkologische Patienten}

Die febrilen hämatoonkologischen Patienten $(\mathrm{n}=10)$ wurden alle mit der Standarddosierung 3-mal täglich $1000 \mathrm{mg}$ Meropenem behandelt. Unter dieser Dosierung wurden wie bereits erwähnt die erforderlichen Wirkspiegel oft frühzeitig unterschritten. Ursächlich für die frühzeitige Unterschreitung effektiver Antibiotikaspiegel war insbesondere die oft stark beschleunigte Elimination von Meropenem. Die totale Meropenem-Clearance war mit durchschnittlich $218 \pm 66 \mathrm{ml} / \mathrm{min}$ etwa so hoch wie bei gesunden jungen Freiwilligen (206 $\mathrm{ml} / \mathrm{min}$ ) (Dreetz et al. 1996). Allerdings lag das Durchschnittsalter der Studienpatienten bei 55 Jahren. Die Meropenem-Clearance war größer als es bei gesunden Patienten der gleichen Altersstufe zu erwarten wäre. Sie überschritt bei einzelnen Patienten mit bis zu $345 \mathrm{ml} / \mathrm{min}$ sogar deutlich die Werte gesunder junger Probanden.

Eine Erhöhung der Clearance von Beta-Lactam-Antibiotika bei Patienten mit malignen hämatologischen Erkrankungen wurde auch von anderen Autoren beobachtet. Nyhlen et al., die die Pharmakokinetik von Meropenem bei febrilen neutropenischen Patienten untersuchten, fanden ebenfalls eine Erhöhung der Meropenem-Clearance in dieser Patienten- 
gruppe. Die totale und renale Clearance $\left(209 \pm 92,4\right.$ und $\left.150 \pm 70,1 \mathrm{ml} / \mathrm{min} \cdot 1,73 \mathrm{~m}^{2}\right)$ wie auch die tubuläre Sekretion ( $49 \pm 41 \mathrm{ml} / \mathrm{min} \cdot 1,73 \mathrm{~m}^{2}$ ) von Meropenem tendierten zu höheren Werten als bei gesunden Freiwilligen (Nyhlen et al. 1997). Deanen et. al. fanden eine Erhöhung der Medikamentenclearance im Vergleich zu gesunden Freiwilligen auch bei der Behandlung febriler hämatoonkologischer Patienten mit dem Beta-Lactam-Antibiotikum Ceftazidim (Daenen et al. 1995). Pea et al. zeigten bei Patienten mit akuter myeloischer Leukämie und febriler Neutropenie, die mit Ceftazidim behandelt wurden, ebenfalls eine erhöhte renale Medikamentenclearance sowie ein erhöhtes Verteilungsvolumen (Pea et al. 2005b).

Bei den von uns untersuchten hämatoonkologischen Patienten bestand eine lineare Korrelation zwischen der Kreatinin-Clearance und der totalen Meropenem-Clearance $(R=0,78)$. Es bestand somit eine formale Abhängigkeit zwischen der glomerulärer Clearance und der Gesamtclearance von Meropenem. Als mögliche Ursachen für die erhöhte Medikamentenclearance von Meropenem kommen verschiedene erkrankungs- und therapiebedingte Veränderungen in Frage. So kommt es im Rahmen von Fieber und Infektion häufig zu einer Hyperzirkulation mit erhöhter renaler Medikamentenausscheidung. Im Rahmen der Therapie erfolgte häufig eine prophylaktische Hyperhydratation und Anwendung von Diuretika als weitere mögliche Ursachen für die erhöhte Meropenem-Clearance.

Das Verteilungsvolumen von Meropenem war im Vergleich zu gesunden Freiwilligen mit $\mathrm{V}_{\mathrm{ss}}=23,31(15,0-37,0) 1$ erhöht und unterlag einer gewissen Variabilität.

Wir fanden bezüglich der Pharmakokinetik von Meropenem bei den von uns untersuchten hämatoonkologischen Patienten relevante Unterschiede zwischen den neutropenischen und nicht-neutropenischen Patienten. Dieser Aspekt wurde in der vorliegenden Literatur bisher nicht beschrieben, muss jedoch angesichts der kleinen Fallzahl in einer größeren Studie validiert werden. So war die Meropenem-Clearance bei den neutropenischen Patienten $(n=6)$ mit $255 \pm 59 \mathrm{ml} / \mathrm{min}$ durchschnittlich $60 \%$ größer $(\mathrm{p}=0,016)$, das Verteilungsvolumen war mit 19,3 $\pm 5,51$ durchschnittlich 50\% kleiner $(\mathrm{p}=0,042)$ als bei den Nicht-Neutropenikern. Eine hohe Meropenem-Clearance, insbesondere in Kombination mit einem relativ niedrigen Verteilungsvolumen, führte gerade bei den Neutropenikern unter den hämatoonkologischen Patienten zur schnelleren Elimination von Meropenem und frühzeitigen Unterschreitung suffizienter Plasmaspiegel.

Die erforderlichen Wirkspiegel zur Bekämpfung der eigentlich hochsensiblen Enterobacteriaceae $(\mathrm{MIC} 90=2 \mathrm{mg} / \mathrm{l})$ fielen bei den neutropenischen Patienten bereits nach durchschnittlich 66\% und zur Bekämpfung des intermediären Pseudomonas aeruginosa (MIC90 $=4 \mathrm{mg} / \mathrm{l}$ ) sogar schon nach 49\% des Dosierungsintervalls unter die angegebenen Hemmkonzentra- 
tionen. Die Minimumkonzentration am Ende des Dosierungsintervalls lag bei den febrilen neutropenischen Patienten bei 0,31 $(<0,01-0,66) \mathrm{mg} / \mathrm{l}$.

Bei den nicht-neutropenischen Patienten wurden die erforderlichen Wirkspiegel (4 und 2mg/l) länger aufrechterhalten. Die angegebenen Hemmkonzentrationen wurden erst nach 89 bzw. 68\% des Dosierungsintervalls unterschritten. Auch die Minimumkonzentration am Ende des Dosierungsintervalls war mit 2,3 (1,6-2,8) mg/l deutlich höher als bei den neutropenischen Patienten.

Zur Bekämpfung hochsensibler Keime scheint eine Meropenem-Dosierung von 3-mal 1000 $\mathrm{mg} / \mathrm{d}$ bei neutropenischen Patienten mit Fieber gerade noch ausreichend zu sein, wohingegen eine suffiziente Wirkung bei intermediären Keimen wie Pseudomonas aeruginosa nicht gewährleistet scheint.

Insbesondere bei den Neutropenikern in unserer Studie war auch im Vergleich zu den NichtNeutropenikern die terminale HWZ $\left(\mathrm{t}_{1 / 2 \beta}\right)$ deutlich verkürzt $(1,14 \pm 0,29 \mathrm{~h}$ vs. $3,23 \pm 1,11 \mathrm{~h} ; \mathrm{p}$ $=0,002)$ und die Fläche unter der Zeit-Konzentrations-Kurve $\left(\mathrm{AUC}_{0-\infty}\right)$ stark vermindert $(68,4$ $\pm 16,5$ vs. $104,3 \pm 11,7 \mathrm{mg} * \mathrm{~h} / \mathrm{l} ; \mathrm{p}=0,006)$.

Ein Extrembeispiel findet sich bei Patient Nr. 1. Bei diesem Patienten bestand Fieber in Neutropenie. Er hatte von allen Patienten mit 345 ml/min die höchste Meropenem-Clearance und mit 15,0 $1(0,231 / \mathrm{kg})$ eines der niedrigsten Verteilungsvolumina von allen hämatoonkologischen Patienten. Bei diesem Patienten kam es somit zur raschen Elimination. Die Plasmaspiegel von Meropenem fielen bereits nach 30\% des Dosierungsintervalls unter die MIC 90 von Pseudomonas aeruginosa (4 mg/l) und nach 35\% des Dosierungsintervalls unter die MIC 90 von Enterobacteriaceae $(2 \mathrm{mg} / \mathrm{l})$. Die Minimumkonzentration am Ende des Dosierungsintervalls lag bei $<0,01 \mathrm{mg} / 1$.

Die Tatsache, dass hämatoonkologische Patienten und hier insbesondere die Neutropeniker oft frühzeitig die minimalen Hemmkonzentrationen klinisch relevanter Keime unterschritten ist besonders relevant, da sie aufgrund ihrer krankheits- und chemotherapieinduzierten Abwehrschwäche am meisten auf eine suffiziente Antibiotikatherapie angewiesen sind. Die neutropenischen hämatoonkologischen Patienten erlitten die schwereren Infektionen und hatten einen schlechteren Outcome als die nicht-neutropenischen Patienten. Unter den 6 neutropenischen Patienten hatten alle Patienten eine Sepsis $(n=3)$ oder wahrscheinliche Sepsis $(n=3)$, wohingegen von den 4 Nicht-Neutropenikern 3 Patienten keine Sepsis und nur 1 Patient lediglich den Verdacht auf eine Sepsis hatten.

Während in der Gruppe der Nicht-Neutropeniker alle Patienten innerhalb von 7 Tagen unter Meropenem entfieberten, gab es in der Gruppe der Neutropeniker 2 Therapieversager, von 
denen ein Patient unter Meropenem nicht entfieberte und ein weiterer Patient an den Folgen der Infektion verstarb.

Diese Daten machen deutlich, dass es unter Anwendung von Meropenem-,,Standarddosierungen" bei hämatoonkologischen Patienten mit Fieber und hier insbesondere bei neutropenischen Patienten häufig zu insuffizienten Medikamentenspiegeln mit der bedrohlichen Konsequenz eines Therapieversagens bzw. eines infausten Ausgangs kommen kann. Patienten, die hinsichtlich ihrer Grunderkrankung eine kurative Heilungschance haben, versterben in signifikanter Zahl an den Folgen einer durch Chemotherapie induzierten Abwehrschwäche und begleitenden Infektion.

\subsubsection{Intensivpatienten}

Die Intensivpatienten mit schweren Infektionen und Sepsis $(\mathrm{n}=15)$ wurden mit den Standarddosierungen 2- bis 3-mal täglich 500 bzw. 1000 mg Meropenem behandelt. In dieser Patientengruppe zeigte sich eine wesentlich größere Variabilität der pharmakokinetischen Parameter als bei den hämatoonkologischen Patienten. Es wurden stark erhöhte Verteilungsvolumina und in Bezug auf die Nierenfunktion unerwartet hohe Meropenem-Clearances gefunden. $\mathrm{Zu}$ ähnlichen Ergebnissen kamen auch andere Autoren, die sich ebenfalls mit der Pharmakokinetik von Meropenem bei kritisch kranken Patienten mit schweren Infektionen beschäftigten. (Bedikian et al. 1994, de Stoppelaar et al. 2000, Giles et al. 2000, Hurst und Lamb 2000, Kitzes-Cohen et al. 2002, Tegeder et al. 1999, Thalhammer et al. 1999).

Insbesondere bei den von uns untersuchten Intensivpatienten ohne wesentliche Nierenfunktionseinschränkung $\left(\mathrm{Cl}_{\text {krea }}>60 \mathrm{ml} / \mathrm{min}\right)$ kam es infolge erhöhter Medikamentenclearance gehäuft zu einer frühzeitigen Unterschreitung der erforderlichen Wirkspiegel von Meropenem. Die Unterschreitung war zum Teil mit einem schlechten klinischen Response verbunden.

Bei Patienten, die im Rahmen einer Sepsis und eines septischen Schocks eine akute Nierenfunktionseinschränkung erlitten, kam es trotz Anpassung der Meropenem-Dosis nach Normogramm an die renale Funktion gehäuft zur Kumulation.

Folgende zentrale Aussagen lassen sich aus den gewonnenen Daten der Intensivpatienten ableiten:

1) Die Intensivpatienten hatten häufig ein stark erhöhtes und interindividuell stark schwankendes Verteilungsvolumen: 
Bei gesunden Freiwilligen liegt das Verteilungsvolumen von Meropenem bei etwa 171 (Bax et al. 1989). Bei den von uns untersuchten Intensivpatienten war das durchschnittliche Verteilungsvolumen von Meropenem im Vergleich zu gesunden Freiwilligen stark erhöht $\left(\mathrm{V}_{\mathrm{ss}}\right.$ $=34,5 \pm 14,5$ 1) und unterlag einer hohen Variabilität. Ähnlich hohe Verteilungsvolumina von Meropenem wurden in früheren Studien bei Patienten mit intraabdominellen Infektionen $\left(\mathrm{V}_{\mathrm{ss}}\right.$ $=26,7 \pm 6,9$ 1) (Bedikian et al. 1994), bei Intensivpatienten mit beatmungsassoziierter Pneumonie $\left(\mathrm{V}_{\mathrm{ss}}=34,4 \pm 15,9\right.$ 1)(de Stoppelaar et al. 2000) sowie bei Intensivpatienten mit akuter Niereninsuffizienz und veno-venöser Hämofiltration $\left(\mathrm{V}_{\mathrm{ss}}=27,9\right.$ 1) (Giles et al. 2000) gefunden.

Die in unserer Studie untersuchten Intensivpatienten mit septischem Schock tendierten zu einem höheren Verteilungsvolumen als die Intensivpatienten ohne septischen Schock $(38,3$ vs. 29,3 1). Der Unterschied war jedoch aufgrund der stark schwankenden Verteilungsvolumina nicht signifikant.

Es bestand auch kein direkter Zusammenhang zwischen der Höhe des Verteilungsvolumens und dem Grad der Nierenfunktionseinschränkung. Diese fehlende Korrelation bei der Behandlung mit Meropenem wurde ebenfalls von Kitzen-Cohen et al. bei kritisch kranken Patienten (Kitzes-Cohen et al. 2002) als auch von Christensson et al. bei Probanden mit verschieden Graden von Nierenfunktionseinschränkungen (Christensson et al. 1992) gesehen. Christensson et al. zeigten sogar, dass der Grad der Nierenfunktionseinschränkung keinen Einfluss auf des Verteilungsvolumen von Meropenem hatte (Christensson et al. 1992).

Das stark erhöhte Verteilungsvolumen ist also nicht ausschließlich durch die häufig auftretende Verminderung der renalen Funktion und folgenden Retention, sondern vielmehr durch infektionsspezifische Abnormalitäten wie Erhöhung der Gefäßpermeabilität oder Ödeme infolge von Infektion und Sepsis verursacht. Wir fanden allerdings aufgrund der großen Variabilität keinen statistischen Zusammenhang zwischen der Schwere der Infektion und der Höhe des Verteilungsvolumens.

2) Bei den Intensivpatienten zeigte sich oft eine höhere Meropenem-Clearance als es die Nierenfunktion erwarten ließ.

Bei den von uns untersuchten nicht-hämatoonkologischen Intensivpatienten überstieg die Meropenem-Clearance die Kreatinin-Clearance um den Faktor 2,9 $\pm 1,3$. Leroy et al. fanden bei gesunden Freiwilligen einen Faktor von 1,5 (Leroy et al. 1992). Durch Bedikian et al. wurde bei Patienten mit intraabdominellen Infektionen ein ähnliches Verhältnis zwischen Meropenem- und Kreatinin-Clearance $(3,0)$ gefunden. In dieser Studie zeigte sich ein 
isolierter Anstieg der extrarenalen Meropenem-Clearance bei unveränderter im Urin gemessener renaler Meropenem-Clearance (Bedikian et al. 1994). Dies deutet darauf hin, dass es zu einer Erhöhung der extrarenalen Clearance bei kritisch kranken Patienten mit schweren Infektionen kommen kann.

Als eine mögliche Erklärung für die vermutete Erhöhung der extrarenalen Clearance kommt die starke Erhöhung des Verteilungsvolumens und somit der mittleren Aufenthaltsdauer (MRT) des Pharmakons im Körper in Frage. Hieraus resultiert eine prolongierte Expositionszeit gegenüber der renalen Dehydropeptidase 1, unspezifischen Hydrolasen und möglicherweise bestimmter postulierter Enzymsysteme im Gewebe. Diese können Meropenem durch Öffnung seines Beta-Lactamrings in seinen inaktiven Metaboliten (ICI 213,689) überführen.

Leroy et al. fanden ein unterschiedlich großes Ausmaß des Metabolismus bei verschiedenen Patientengruppen. Die Konzentration des Metaboliten im Plasma war bei gesunden Freiwilligen relativ niedrig. Hingegen fanden sich bei urämischen Patienten, die ähnlich wie die von uns untersuchten Intensivpatienten ein erhöhtes Verteilungsvolumen aufwiesen, signifikant erhöhte Metabolitenkonzentrationen (Leroy et al. 1992). Der genaue Anteil des Meropenem-Metabolismus an der Meropenem-Clearance wurde in der vorliegenden Studie jedoch nicht gemessen.

3) Bei den Intensivpatienten bestand keine statistische Korrelation zwischen MeropenemClearance und Kreatinin-Clearance:

Im Gegensatz zu den Ergebnissen der von uns untersuchten hämatoonkologischen Patienten und auch im Widerspruch zu den Beobachtungen anderer Autoren (Bax et al. 1989, Chimata et al. 1993, Christensson et al. 1992, Leroy et al. 1992), die sich mit der Pharmakokinetik von Meropenem in Bezug auf verschiedene Grade der Nierenfunktionseinschränkung beschäftigten, bestand in der Gruppe der Intensivpatienten keine lineare Korrelation zwischen Kreatinin- und Meropenem-Clearance $(\mathrm{R}=0,36)$. Die Elimination von Meropenem war also nicht allein von der glomerulären Funktion abhängig, sondern auch durch andere Faktoren stark beeinflusst. Ähnliche Beobachtungen machten auch Bedikian et al. bei Patienten mit intraabdominellen Infektionen. In dieser Patientengruppe bestand ebenfalls keine lineare Korrelation zwischen Meropenem- und Kreatinin-Clearance (Bedikian et al. 1994).

Als Ursache für den fehlenden Zusammenhang zwischen Meropenem- und KreatininClearance bei den Intensivpatienten mit schweren Infektionen sehen wir die starke Variabilität der Verteilungsvolumina. Als zusätzliche Ursache könnte eine potentiell interindividuell variierende extrarenale Meropenem-Clearance durch Hydrolyse oder enzymatischen Abbau in 
Frage kommen. Ein solcher Abbau wurde insbesondere für Imipenem aber auch für andere Carbapeneme beschrieben.

Während die Pharmakokinetik bei den hämatoonkologischen Patienten überwiegend von der Nierenfunktion bestimmt wurde, spielten bei den Intensivpatienten darüber hinaus eine erhöhte Variabilität der Verteilungsvolumina und eine möglicherweise zusätzlich vorhandene variierende extrarenale Clearances eine entscheidende Rolle für die interindividuell stark schwankenden pharmakokinetischen Parameter.

4) Bei den Intensivpatienten bestanden große Meropenem-Plasmaspiegelfluktuationen:

Aufgrund der großen Variabilität der Pharmakokinetik infolge einer variierenden renalen Clearance, einem hohem und stark schwankenden Verteilungsvolumen sowie einem eventuell vorhandenen, gesteigerten Metabolismus kam es bei den von uns untersuchten Intensivpatienten zu interindividuell starken Plasmaspiegelfluktuationen.

Die gefundenen Maximumkonzentrationen $\left(\mathrm{C}_{\max }\right)$ unterschieden sich um einen Faktor 7, was auf die starken Schwankungen des Verteilungsvolumens, aber auch auf die unterschiedlichen Meropenem-Dosen (500 mg bzw. 1000 mg) zurückzuführen war. Die Minimumkonzentrationen unterschieden sich um einen Faktor größer als 700. Bei einigen Patienten kam es zur frühzeitigen Unterschreitung suffizienter Plasmaspiegel, bei anderen Patienten infolge verzögerter Elimination bei stark verminderter Nierenfunktion zur Kumulation. Die Fläche unter der Zeit-Konzentrations-Kurve (AUC) folgte der großen interindividuellen Variabilität und schwankte um den Faktor 18 (18,2-327,8 $(\mathrm{mg} \cdot \mathrm{h}) / \mathrm{l})$. Sie war bei den Intensivpatienten ohne Nierenfunktionseinschränkung mit $68,5(\mathrm{mg} \cdot \mathrm{h}) / 1$ vergleichbar groß wie bei gesunden Freiwilligen (70,5 mg / $(1 \cdot h)$ ) (Dreetz et al. 1996) und bei den Intensivpatienten mit Nierenfunktionseinschränkung deutlich erhöht $(160,5 \mathrm{mg} /(1 \cdot \mathrm{h}))$.

\section{Intensivpatienten ohne Nierenfunktionseinschränkung}

Die insgesamt 6 Intensivpatienten (Durchschnittsalter 53 J.) ohne Nierenfunktionseinschränkung $\left(\mathrm{Cl}_{\text {krea }}>60 \mathrm{ml} / \mathrm{min}\right)$ wurden nach Normogramm mit den Standarddosierungen 3-mal täglich 500 bzw. 1000 mg Meropenem behandelt. Sie unterschritten die Hemmkonzentrationen klinisch relevanter Keime oft frühzeitig. Die MIC90 für Enterobacteriaceae (2 mg/l) wurde nach durchschnittlich 73\% (31-100\%), die MIC90 für Pseudomonas aeruginosa (4 mg/l) nach durchschnittlich 56\% (11-100\%) des Dosierungsintervalls unterschritten. Bei einem von drei Patienten mit der Dosierung 3-mal 1000 mg/d und bei allen 3 Patienten mit der Dosierung 3-mal 500 mg/d wurden die Hemmkonzentrationen für intermediäre Keime (4 mg/l) nach weniger als $60 \%$ des Dosierungsintervalls unterschritten. Unter den 4 Patienten 
mit frühzeitiger Unterschreitung erforderlicher Wirkspiegel konnte bei 2 Patienten aufgrund hochsensibler Keime $(\mathrm{MIC}=0,25)$ eine Entfieberung erreicht werden, bei den anderen 2 Patienten kam es bei frühzeitig niedrigen Spiegeln zum Therapieversagen.

Als Ursache für den frühzeitigen Meropenem-Plasmaspiegelabfall wurde bei den Patienten ohne Nierenfunktionseinschränkung neben der relativ guten renal-glomerulären Ausscheidung $\left(\mathrm{Cl}_{\mathrm{krea}}=80,8 \mathrm{ml} / \mathrm{min}(63,3-114,0 \mathrm{ml} / \mathrm{min})\right)$ eine unerwartet hohe Meropenem-Clearance gefunden. Die Meropenem-Clearance betrug bei den Intensivpatienten ohne Nierenfunktionseinschränkung 225,6 $\pm 114,5 \mathrm{ml} / \mathrm{min}$ und überstieg die Kreatinin-Clearance um den Faktor 2,8. Sie war somit größer als bei jungen gesunden Probanden (206 ml/min) (Krueger et al. 2005). Im Einzelfall wurde dieser Wert mit bis zu $399 \mathrm{ml} / \mathrm{min}$ deutlich überschritten. Dies deckt sich mit den Ergebnissen von Roberts et al., die bei kritisch kranken Patienten mit Sepsis ohne Nierenfunktionseinschränkung eine durchschnittliche Meropenem-Clearance von $227 \mathrm{ml} / \mathrm{min}$ fanden (Roberts et al. 2009).

Im Vergleich zu ähnlichen Studien überschritt die Meropenem-Clearance der von uns untersuchten Intensivpatienten die Werte von Patienten mit beatmungsassoziierter Pneumonie $\left(\mathrm{Cl}_{\text {mer }} 183 \pm 71,7 \mathrm{ml} / \mathrm{min}\right.$ ) (de Stoppelaar et al. 2000). Sie war allerdings durchschnittlich kleiner als bei Patienten mit intraabdominellen Infektionen $\left(\mathrm{Cl}_{\mathrm{mer}}=315,4 \pm 71,9 \mathrm{ml} / \mathrm{min}\right)$ (Bedikian et al. 1994).

Das durchschnittliche Verteilungsvolumen war bei den von uns untersuchten Intensivpatienten ohne Nierenfunktionseinschränkung mit durchschnittlich 33,6 1 stark erhöht und unterlag einer hohen Variabilität (19,5-69,4 1).

Als Beispiel für die pharmakokinetischen Zusammenhänge bei den Intensivpatienten ohne Nierenfunktionseinschränkung sei Patient Nr. 11 genannt. Dieser Patient hatte infolge einer dekompensierten Herzinsuffizienz eine Stauungspneumonie mit Sepsis. Die KreatininClearance betrug 78,8 ml/min. Die Meropenem-Clearance lag bei 311,3 ml/min. Sie überstieg die renale Kreatinin-Clearance somit um den Faktor 3,9. Das Verteilungsvolumen betrug 19,5 1. Die MIC90 für Enterobacteriaceae (2 mg/l) wurde bereits nach 40\% des Dosierungsintervalls und die MIC90 für Pseudomonas aeruginosa (4 mg/l) sogar schon nach 30\% des Dosierungsintervalls unterschritten.

\section{Intensivpatienten mit Nierenfunktionseinschränkung}

Bei den Intensivpatienten, insbesondere bei denen mit Sepsis und septischem Schock kam es gehäuft zur Nierenfunktionseinschränkung. Die Intensivpatienten mit septischem Schock tendierten zu einer schlechteren Nierenfunktion als die Patienten ohne septischen Schock. Bei 
diesen Patienten war die renale Elimination von Meropenem verzögert, bei hochgradiger Nierenfunktionseinschränkung zeigte sich häufiger eine Kumulation.

Unter den 9 Intensivpatienten mit Nierenfunktionseinschränkung $\left(\mathrm{Cl}_{\text {krea }}<60 \mathrm{ml} / \mathrm{min}\right)$ waren 6 Patienten bei denen ein Nierenersatzverfahren durchgeführt werden musste. Bei 4 dieser Patienten wurde zum Untersuchungszeitpunkt aufgrund eines akuten Nierenversagens eine kontinuierliche veno-venöse Hämofiltration (CVVHF) durchgeführt und bei 2 Patienten bestand ein unmittelbarer Zustand nach CVVHF. Bei diesen Patienten konnte die KreatininClearance nicht aus den Serumkreatininspiegeln abgeschätzt werden. Bei den 3 Intensivpatienten mit Nierenfunktionseinschränkung ohne Nierenersatztherapie lag die errechnete Kreatinin-Clearance bei $36,6 \pm 23,5 \mathrm{ml} / \mathrm{min}$.

Wie erwartet unterschritten die Intensivpatienten mit Nierenfunktionseinschränkung aufgrund der verzögerten renalen Elimination die Hemmkonzentrationen relevanter Keime relativ spät. Sie hatten somit anhaltend hohe therapeutische Spiegel. Die MIC90 für Enterobacteriaceae (2 mg/l) wurde nach durchschnittlich 99\% (94 - 100\%) und die MIC90 für Pseudomonas aeruginosa (4 mg/l) nach durchschnittlich 86\% (60 - 100\%) des Dosierungsintervalls unterschritten. Bei allen Patienten überstiegen die Plasmaspiegel von Meropenem während mehr als 60 $70 \%$ des Dosierungsintervalls die erforderlichen Hemmkonzentrationen.

Die Minimumkonzentrationen am Ende des Dosierungsintervalls lagen bei 6,9 mg/l (2,3 14,2 mg/l). Bei insgesamt 3 Patienten kam es zur Kumulation mit Spiegeln größer 10 mg/l am Ende des Dosierungsintervalls.

Sowohl die Dosierung mit 3-mal 500 mg Meropenem pro Tag, als auch die Dosierung mit 2mal 1000 mg Meropenem pro Tag führten bei Patienten mit Nierenfunktionseinschränkungen zu suffizienten Plasmaspiegel.

Die Meropenem-Gesamtclearance war entsprechend der Nierenfunktionseinschränkung erniedrigt und betrug $97 \pm 44,4$ ml/min. Ähnliche Beobachtungen machten Kitzes-Cohen etal. bei kritisch kranken Patienten mit Nierenfunktionsstörungen $\left(\mathrm{Cl}_{\text {krea }}<50 \mathrm{ml} / \mathrm{min}\right)$. Bei diesen Patienten wurde eine Meropenem-Gesamtclearance von 77,7 $\pm 15,8 \mathrm{ml} / \mathrm{min}$ gefunden (KitzesCohen et al. 2002).

Das Verteilungsvolumen von Meropenem bei den Intensivpatienten mit Nierenfunktionseinschränkung lag bei 35,1 $\pm 12,51(0,45 \pm 0,13 \mathrm{l} / \mathrm{kg})$ und war somit stark erhöht mit großer Variabilität. Es war jedoch vergleichbar mit den Verteilungsvolumina bei Intensivpatienten ohne Nierenfunktionseinschränkungen (33,6 1). Wie bereits erwähnt hatte das Ausmaß der Nierenfunktionseinschränkung keinen wesentlichen Einfluss auf die Größe des Verteilungsvolumens. Vielmehr scheint sich zu bestätigen, dass infektionsspezifiche Abnormalitäten, wie 
erhöhte Gefäßpermeabilität und Ödeme einen größeren Einfluss auf das Verteilungsvolumen haben als die Nierenfunktion.

Bei 3 der 9 Intensivpatienten mit Nierenfunktionseinschränkung war der Outcome gut bis befriedigend mit Entfieberung unter Meropenem innerhalb von 7 Tagen. Bei 2 Patienten kam es trotz suffizienter Meropenem-Plasmaspiegel aufgrund hochresistenter Keime zum Therapieversagen ohne Entfieberung. 4 Patienten verstarben infolge anderer Ursache als Infektion.

\subsection{Therapeutische Konsequenzen}

Die Integration pharmakokinetischer und mikrobiologischer Daten kann zur Erstellung rationaler Dosierungsstrategien von Antibiotika beitragen. Ziel jeglicher Dosierungsstrategie ist das Erreichen maximaler bakterizider Wirkung unter Vermeidung von Resistenzentwickung und von unerwünscht toxischen Effekten. Meropenem ist ein BreitspektrumAntibiotikum mit zeitabhängiger Wirkung. Der entscheidende pharmakokinetische Parameter für die Wirkintensität ist die Dauer in der der Plasmaspiegel des Therapeutikums die minimale Hemmkonzentration des zu bekämpfenden Keims überschreitet (\%T > MIC). Es wird empfohlen, dass die minimale Hemmkonzentration des zu bekämpfenden Keims während mindestens 60-70\% des Dosierungsintervalls überschritten wird (Cars 1997, Craig 1998, Turnidge 1998). Bei kritisch kranken Patienten wird sogar eine Überschreitung der minimalen Hemmkonzentration des zu bekämpfenden Keims während des gesamten Dosierungsintervalls empfohlen (Lamoth et al. 2009, Pea und Viale 2006). Die häufig vorkommenden Keime in den untersuchten Patientengruppen haben eine minimale Hemmkonzentration von 2-4 mg/l. Die Meropenem-Minimumkonzentration am Ende des Dosierungsintervalls zur Gewährleistung einer effektiven Therapie diese Konzentration überschreiten. Durch Optimierung der Dosierungsschemata lässt sich dieses pharmakodynamische Ziel erreichen.

Der Plasmakonzentrationsverlauf von Meropenem ist abhängig:

1) von der applizierten Dosis: je höher die Dosis desto länger die Zeiten über der MIC

2) von der Dauer des Dosierungsintervalls: je kürzer das Dosierungsintervall, desto größer sind die Zeiträume über der MIC

3) von der Applikationsweise: Bolus vs. prolongierte Infusion vs. kontinuierliche Infusion. Im Rahmen populationskinetischer Modelle konnte gezeigt werden, dass eine Verlängerung der Infusion von $1000 \mathrm{mg}$ Meropenem von $30 \mathrm{~min}$ auf 3 h, die Zeit über der MIC von 64 auf 90\% erhöhte (Li et al. 2006). 
4) von der Meropenem-Clearance des individuellen Patienten: je größer die Clearance, desto schneller fallen die Plasmaspiegel unter die MIC

5) vom Verteilungsvolumen: je größer die Verteilungsräume, desto höher die terminalen Plasmaspiegel.

Die therapeutisch beeinflussbaren Faktoren sind die Dosis, die Dosierungsintervalle und die Applikationsart. Sie müssen auf die individuellen Clearances und Verteilungsräume abgestimmt werden.

Bei Patienten mit hoher Clearance und niedrigen Verteilungsvolumen kommt es zu einer frühen Unterschreitung der minimalen Hemmkonzentration des zu bekämpfenden Keims. Bei diesen Patienten ist eine Dosiserhöhung und/oder Verkürzung des Dosierungsintervalls zu empfehlen. Einige Autoren empfehlen auch eine prolongierte oder kontinuierliche Infusion von Carbapenemen um ausreichende Konzentrationen über der MIC des zu bekämpfenden Keims während des gesamten Dosierungsintervalls zu gewährleisten (Lamoth et al. 2009, Thalhammer et al. 1999).

Die hämatoonkologischen Patienten und hier insbesondere die febrilen Neutropeniker, als auch die nicht-hämatoonkologischen Intensivpatienten ohne wesentliche Nierenfunktionseinschränkung mit schweren Infektionen unterschritten die erforderlichen Wirkkonzentrationen frühzeitig. Diese Ergebnisse belegen die Notwendigkeit von Plasmaspiegelmessungen und einer individuellen Dosisanpassung, um eine ausreichende Zielkonzentrationen aufrecht zu erhalten und somit eine wirksame Therapie zu gewährleisten. Denkbar wäre eine Messung der Minimumkonzentration vor Gabe der nächsten Meropenem-Dosis, welche insbesondere bei immuninkompetenten Patienten die minimale Hemmkonzentration der erwarteten Keime von 2-4 mg/l überschreiten sollte. Eine ähnliche Methode wurde bereits für Imipenem durchgeführt (Lamoth et al. 2009). Eine Obergrenze für die Meropenem-Konzentration ist in der vorliegenden Literatur nicht definiert.

Therapeutisches Drugmonitoring und Dosisanpassung in Form von Verkürzung der Dosierungsintervalle, Erhöhung der Meropenem-Dosen und/oder Modifizierung der Applikationsweise sollten in diesen Patientengruppen erwogen werden. 


\section{$5 \quad$ Zusammenfassung}

Meropenem ist ein Carbapenem-Antibiotikum mit breitem Wirkspektrum, guter Verträglichkeit und hoher Gewebepenetration, auch in infiziertes Gewebe. Obwohl Meropenem aufgrund seiner guten pharmakokinetischen und pharmakodynamischen Eigenschaften häufig zur empirischen Therapie kritisch kranker Patienten mit schweren Infektionen zum Einsatz kommt, ist über seine Pharmakokinetik bei diesen Patienten nur wenig bekannt. Die aktuell vorliegenden Dosierungsempfehlungen beruhen überwiegend auf pharmakokinetischen Studien an gesunden Probanden oder Patienten mit leichten Infektionen. Studien mit kritisch kranken Risikopatienten liegen nur wenige vor.

In der vorliegenden Arbeit wurde die Pharmakokinetik von Meropenem bei ausgewählten hämatoonkologischen Patienten mit Fieber $(n=10)$ und bei nicht-hämatoonkologischen Intensivpatienten mit schweren Infektionen $(n=15)$ untersucht. In 25\% der Fälle konnte unter Anwendung von Meropenem-,,Standarddosierungen“"keine Entfieberung erreicht werden. Als eine potentielle Ursache für das Therapieversagen wurde häufig eine frühzeitige Unterschreitung effektiver Meropenem-Wirkspiegel infolge veränderter Pharmakokinetik im Rahmen der vorliegenden Infektion gefunden.

Die Zeit, in der die Plasmakonzentration die MIC (minimale Hemmkonzentration) des zu bekämpfenden Erregers übersteigt, ist der pharmakokinetische Parameter, der die antibakterielle Aktivität von Beta-Lactam-Antibiotika in vivo am besten vorhersagt. Für Meropenem wird empfohlen, dass bei potentiell lebensbedrohlichen Erkrankungen die Antibiotikakonzentration während 70-100\% des Dosierungsintervalls über der MIC des zu bekämpfenden Keims liegt. Die angestrebte MIC der häufigsten in den untersuchten Patientengruppen vorkommenden Keime von $4 \mathrm{mg} / \mathrm{l}$ wurde jedoch unter Anwendung der empfohlenen Standarddosierungen bei $56 \%$ der Patienten nach weniger als $70 \%$ des Dosierungsintervalls unterschritten.

Bei den febrilen hämatoonkologischen Patienten beruhte die oft frühzeitige Unterschreitung erforderlicher Meropenem-Wirkspiegel hauptsächlich auf der oft unerwartet hohen Meropenem-Clearance $(217 \mathrm{ml} / \mathrm{min})$, welche eng mit der endogenen Kreatinin-Clearance korrelierte $(\mathrm{R}=0,78)$, und so auf eine erhöhte renale Elimination zurückgeführt werden konnte. Insbesondere bei den hämatoonkologischen Patienten mit Neutropenie war eine ausgeprägte Meropenem-Clearance $(255 \mathrm{ml} / \mathrm{min}$ ) zu beobachten. Bei diesen Patienten wurden die Mindestkonzentrationen zur Bekämpfung der eigentlich hochsensiblen Enterobacteriaceae (MIC90 = $2 \mathrm{mg} / \mathrm{l})$ bereits nach durchschnittlich $66 \%$ und der intermediären Pseudomonas aeruginosa (MIC90 = $4 \mathrm{mg} / \mathrm{l})$ bereits nach durchschnittlich $49 \%$ des Dosierungsintervalls unterschritten. Das Ziel einer Überschreitung der angegebenen minimalen Hemmkonzen- 
trationen während des gesamten Dosierungsintervalls wurde unter Standarddosierung bei keinem der hämatoonkologischen Patienten erreicht. Dies stellte die wahrscheinlichste Ursache eines in $20 \%$ der Fälle aufgetretenen Therapieversagens dar. Hämatoonkologische Patienten, die bezüglich ihrer Grunderkrankung eine kurative Heilungschance haben, erleiden aufgrund ihrer erkrankungs- oder chemotherapiebedingten Abwehrschwäche gehäuft schwere Infektionen mit hoher Mortalität. Sie sind durch insuffiziente Antibiotikatherapie, wie sie in der vorliegenden Studie gehäuft beobachtet wurde, vital bedroht. Die Ergebnisse sollten Anlass zur Therapiekontrolle in Form von Plasmaspiegelmessungen und ggf. Dosierungsmodifikation insbesondere bei kritisch kranken neutropenischen Patienten geben.

Die interindividuelle Variabilität der pharmakokinetischen Parameter in der Gruppe der nichthämatoonkologischen Intensivpatienten mit schweren Infektionen war deutlich größer als bei den hämatoonkologischen Patienten. Es wurden stärkere interindividuelle Fluktuationen der Meropenem-Konzentrationen gesehen. Bei den Intensivpatienten wurde eine interindividuell stark schwankende Nierenfunktion $\left(\mathrm{Cl}_{\text {krea }}=10,4-114 \mathrm{ml} / \mathrm{min}\right)$ mit variierender renaler Clearance, sowie eine starke Erhöhung und große Variabilität der Verteilungsvolumina $\left(\mathrm{V}_{\mathrm{ss}}=\right.$ $34,5 \pm 15,9$ 1) gefunden, welche auch unabhängig von der Nierenfunktion auftraten. Die Meropenem-Clearance war häufig deutlich größer als es die Nierenfunktion erwarten ließ, was möglicherweise auf eine erhöhte extrarenale Clearance zurückzuführen war. Diese Faktoren beeinflussten den Plasmakonzentrationsverlauf von Meropenem in oft nicht vorhersehbarer Weise. Insbesondere die Intensivpatienten ohne Nierenfunktionseinschränkung $\left(\mathrm{Cl}_{\text {krea }}>60 \mathrm{ml} / \mathrm{min}\right)$ hatten eine deutlich erhöhte Meropenem-Clearance $(225 \mathrm{ml} / \mathrm{min})$. Bei diesen Patienten wurde die MIC90 von Enterobacteriaceae $(2 \mathrm{mg} / 1)$ nach 73\% und die MIC90 von Pseudomonas aeruginosa $(4 \mathrm{mg} / \mathrm{l})$ bereits nach 56\% des Dosierungsintervalls unterschritten. Auch hier kam es gehäuft zu einem Therapieversagen ohne Entfieberung unter Meropenem (27\%). Im Rahmen der systemischen Reaktion bei Sepsis und septischem Schock entwickelten viele Intensivpatienten (60\%) eine Nierenfunktionseinschränkung. Diese Patienten zeigten unter der „Standarddosierung“ häufig eine Kumulation von Meropenem mit Minimumkonzentrationen $>10 \mathrm{mg} / \mathrm{l}$.

Auch die Intensivpatienten in unserer Studie könnten im Sinne der Therapiesicherheit von einer Dosisanpassung profitieren. Durch Messungen der Meropenem-Plasmakonzentrationen wären extreme Kumulation und insbesondere unzureichend niedrige Plasmakonzentrationen durch individualisierte Dosierung vermeidbar. 


\section{$6 \quad$ Literaturverzeichnis}

Ariano RE, Nyhlen A, Donnelly JP, Sitar DS, Harding GK, Zelenitsky SA (2005):

Pharmacokinetics and pharmacodynamics of meropenem in febrile neutropenic patients with bacteremia. Ann Pharmacother $\underline{39}, 32-38$

Bax RP, Bastain W, Featherstone A, Wilkinson DM, Hutchison M, Haworth SJ (1989): The pharmacokinetics of meropenem in volunteers. J Antimicrob Chemother 24, 311-320

Bedikian A, Okamoto MP, Nakahiro RK, Farino J, Heseltine PN, Appleman MD, Yellin AE, Berne TV, Gill MA (1994): Pharmacokinetics of meropenem in patients with intra-abdominal infections. Antimicrob Agents Chemother $\underline{38}$, 151-154

Behre G, Link H, Maschmeyer G, Meyer P, Paaz U, Wilhelm M, Hiddemann W (1998): Meropenem monotherapy versus combination therapy with ceftazidime and amikacin for empirical treatment of febrile neutropenic patients. Ann Hematol $\underline{76}, 73-80$

Blumer JL, Reed MD, Kearns GL, Jacobs RF, Gooch WM, III, Yogev R, Willims K, Ewing BJ (1995): Sequential, single-dose pharmacokinetic evaluation of meropenem in hospitalized infants and children. Antimicrob Agents Chemother 39, 1721-1725

Bodmann KF, Vogel F, et al (2001): Antimikrobielle Therapie der Sepsis. Chemother J 10(2), $43-55$

Bone RC, Balk RA, Cerra FB, Dellinger RP, Fein AM, Knaus WA, Schein RM, Sibbald WJ (1992): Definitions for sepsis and organ failure and guidelines for the use of innovative therapies in sepsis. The ACCP/SCCM Consensus Conference Committee. American College of Chest Physicians/Society of Critical Care Medicine. Chest 101, 1644-1655

Boucher BA, Wood GC, Swanson JM (2006): Pharmacokinetic changes in critical illness. Crit Care Clin 22, 255-71

Bowker KE, Holt HA, Reeves DS, MacGowan AP (1996): Bactericidal activity, post antibiotic effect and modified controlled effective regrowth time of meropenem at high concentrations. J Antimicrob Chemother $\underline{38}$, 1055-1060

Brismar B, Malmborg AS, Tunevall G, Lindgren V, Bergman L, Mentzing LO, Nystrom PO, Ansehn S, Backstrand B, Skau T, . (1995): Meropenem versus imipenem/cilastatin in the treatment of intra- abdominal infections. J Antimicrob Chemother $\underline{35}$, 139-148

Byl B, Jacobs F, Roucloux I, de Franquen P, Cappello M, Thys JP (1999): Penetration of meropenem in lung, bronchial mucosa, and pleural tissues. Antimicrob Agents Chemother 43 , $681-682$

Cars O (1981): Tissue distribution of beta-lactam antibiotics. Experimental studies in rabbits. Scand J Infect Dis $\underline{27}, 1-48$

Cars O (1997): Efficacy of beta-lactam antibiotics: integration of pharmacokinetics and pharmacodynamics. Diagn Microbiol Infect Dis 27, 29-33

Chimata M, Nagase M, Suzuki Y, Shimomura M, Kakuta S (1993): Pharmacokinetics of meropenem in patients with various degrees of renal function, including patients with endstage renal disease. Antimicrob Agents Chemother 37, 229-233 
Christensson BA, Nilsson-Ehle I, Hutchison M, Haworth SJ, Oqvist B, Norrby SR (1992): Pharmacokinetics of meropenem in subjects with various degrees of renal impairment. Antimicrob Agents Chemother $\underline{36}$, 1532-1537

Colardyn F, Faulkner KL (1996): Intravenous meropenem versus imipenem/cilastatin in the treatment of serious bacterial infections in hospitalized patients. Meropenem Serious Infection Study Group. J Antimicrob Chemother $\underline{38}$, 523-537

Cometta A, Glauser MP (1996): Empiric antibiotic monotherapy with carbapenems in febrile neutropenia: a review. J Chemother $\underline{8}, 375-381$

Cometta A, Calandra T, Gaya H, Zinner SH, de Bock R, Del Favero A, Bucaneve G, Crokaert F, Kern WV, Klastersky J, Langenaeken I, Micozzi A, Padmos A, Paesmans M, Viscoli C, Glauser MP (1996): Monotherapy with meropenem versus combination therapy with ceftazidime plus amikacin as empiric therapy for fever in granulocytopenic patients with cancer. The International Antimicrobial Therapy Cooperative Group of the European Organization for Research and Treatment of Cancer and the Gruppo Italiano Malattie Ematologiche Maligne dell'Adulto Infection Program. Antimicrob Agents Chemother $\underline{40}$, $1108-1115$

Craig WA (1998): Pharmacokinetic/pharmacodynamic parameters: rationale for antibacterial dosing of mice and men. Clin Infect Dis $\underline{26}, 1-10$

Daenen S, Erjavec Z, Uges DR, Vries-Hospers HG, De Jonge P, Halie MR (1995): Continuous infusion of ceftazidime in febrile neutropenic patients with acute myeloid leukemia. Eur J Clin Microbiol Infect Dis $\underline{14}$, 188-192

de la Camara R., Figuera A, Sureda A, Hermida G, Verge G, Olalla I, Fernandez Ranada JM, Domingo AA (1997): Meropenem versus ceftazidime plus amikacin in the treatment of febrile episodes in neutropenic patients: a randomized study. Haematologica $\underline{82,668-675}$

de Stoppelaar F, Stolk L, van Tiel F, Beysens A, van Der GS, de Leeuw P (2000): Meropenem pharmacokinetics and pharmacodynamics in patients with ventilator-associated pneumonia. J Antimicrob Chemother $\underline{46}, 150-151$

Dehne MG, Kroh UF (1995): Imipenem/cilastin dosage during acute renal failure and hemofiltration. Intensive Care Med 21, 863

Dreetz M, Hamacher J, Eller J, Borner K, Koeppe P, Schaberg T, Lode H (1996): Serum bactericidal activities and comparative pharmacokinetics of meropenem and imipenemcilastatin. Antimicrob Agents Chemother 40, 105-109

Drusano GL, Lode H, Edwards JR (2000): Meropenem: clinical response in relation to in vitro susceptibility. Clin Microbiol Infect $\underline{6}, 185-194$

Edwards JR (1995): Meropenem: a microbiological overview. J Antimicrob Chemother $\underline{36}$, 117

Feld R, DePauw B, Berman S, Keating A, Ho W (2000): Meropenem versus ceftazidime in the treatment of cancer patients with febrile neutropenia: a randomized, double-blind trial. J Clin Oncol 18, 3690-3698

Fish DN, Singletary TJ (1997): Meropenem, a new carbapenem antibiotic. Pharmacotherapy $\underline{17}, 644-669$ 
Forrest A, Nix DE, Ballow CH, Goss TF, Birmingham MC, Schentag JJ (1993):

Pharmacodynamics of intravenous ciprofloxacin in seriously ill patients. Antimicrob Agents Chemother 37, 1073-1081

Freeman CD, Nicolau DP, Belliveau PP, Nightingale CH (1997): Once-daily dosing of aminoglycosides: review and recommendations for clinical practice. J Antimicrob Chemother $\underline{39}, 677-686$

Fuentes F, Martin MM, Izquierdo J, Gomez-Lus ML, Prieto J (1995): In vivo and in vitro study of several pharmacodynamic effects of meropenem. Scand J Infect Dis 27 , 469-474

Giles LJ, Jennings AC, Thomson AH, Creed G, Beale RJ, McLuckie A (2000):

Pharmacokinetics of meropenem in intensive care unit patients receiving continuous venovenous hemofiltration or hemodiafiltration. Crit Care Med 28, 632-637

Gomez CM, Cordingly JJ, Palazzo MG (1999): Altered pharmacokinetics of ceftazidime in critically ill patients. Antimicrob Agents Chemother 43, 1798-1802

Goossens H (2000): MYSTIC (Meropenem Yearly Susceptibility Test Information Collection) results from Europe: comparison of antibiotic susceptibilities between countries and centre types. MYSTIC Study Group (European centres only). J Antimicrob Chemother $\underline{46}, 39-52$

Hanberger H, Svensson E, Nilsson LE, Nilsson M (1995): Control-related effective regrowth time and post-antibiotic effect of meropenem on gram-negative bacteria studied by bioluminescence and viable counts. J Antimicrob Chemother $\underline{35}, 585-592$

Hurst M, Lamb HM (2000): Meropenem: a review of its use in patients in intensive care. Drugs 59, 653-680

Hutchison M, Faulkner KL, Turner PJ, Haworth SJ, Sheikh W, Nadler H, Pitkin DH (1995): A compilation of meropenem tissue distribution data. J Antimicrob Chemother $\underline{36}$, 43-56

Hyatt JM, McKinnon PS, Zimmer GS, Schentag JJ (1995): The importance of pharmacokinetic/pharmacodynamic surrogate markers to outcome. Focus on antibacterial agents. Clin Pharmacokinet 28, 143-160

Jarvis WR, Edwards JR, Culver DH, Hughes JM, Horan T, Emori TG, Banerjee S, Tolson J, Henderson T, Gaynes RP (1991): Nosocomial infection rates in adult and pediatric intensive care units in the United States. National Nosocomial Infections Surveillance System. Am J Med 91, 185-191

Joukhadar C, Frossard M, Mayer BX, Brunner M, Klein N, Siostrzonek P, Eichler HG, Muller M (2001): Impaired target site penetration of beta-lactams may account for therapeutic failure in patients with septic shock. Crit Care Med 29, 385-391

Karjagin J, Lefeuvre S, Oselin K, Kipper K, Marchand S, Tikkerberi A, Starkopf J, Couet W, Sawchuk RJ (2008): Pharmacokinetics of meropenem determined by microdialysis in the peritoneal fluid of patients with severe peritonitis associated with septic shock. Clin Pharmacol Ther $\underline{83}, 452-459$

Kelly HC, Hutchison M, Haworth SJ (1995): A comparison of the pharmacokinetics of meropenem after administration by intravenous injection over 5 min and intravenous infusion over $30 \mathrm{~min}$. J Antimicrob Chemother $\underline{36}, 35-41$ 
Kitzes-Cohen R, Farin D, Piva G, Myttenaere-Bursztein SA (2002): Pharmacokinetics and pharmacodynamics of meropenem in critically ill patients. Int J Antimicrob Agents $\underline{19}, 105-$ 110

Klastersky J (1998): Science and pragmatism in the treatment and prevention of neutropenic infection. J Antimicrob Chemother $\underline{41}, 13-24$

Krueger WA, Bulitta J, Kinzig-Schippers M, Landersdorfer C, Holzgrabe U, Naber KG, Drusano GL, Sorgel F (2005): Evaluation by monte carlo simulation of the pharmacokinetics of two doses of meropenem administered intermittently or as a continuous infusion in healthy volunteers. Antimicrob Agents Chemother 49, 1881-1889

Lamoth F, Buclin T, Csajka C, Pascual A, Calandra T, Marchetti O (2009): Reassessment of recommended imipenem doses in febrile neutropenic patients with hematological malignancies. Antimicrob Agents Chemother $\underline{53}$, 785-787

Leroy A, Fillastre JP, Borsa-Lebas F, Etienne I, Humbert G (1992): Pharmacokinetics of meropenem (ICI 194,660) and its metabolite (ICI 213,689) in healthy subjects and in patients with renal impairment. Antimicrob Agents Chemother $\underline{36}$, 2794-2798

Li C, Kuti JL, Nightingale CH, Nicolau DP (2006): Population pharmacokinetic analysis and dosing regimen optimization of meropenem in adult patients. J Clin Pharmacol $\underline{46}, 1171-1178$

Link H, Bohme A, Cornely OA, Hoffken K, Kellner O, Kern WV, Mahlberg R, Maschmeyer G, Nowrousian MR, Ostermann H (2003): Antimicrobial therapy of unexplained fever in neutropenic patients-guidelines of the Infectious Diseases Working Party (AGIHO) of the German Society of Hematology and Oncology (DGHO), Study Group Interventional Therapy of Unexplained Fever, Arbeitsgemeinschaft Supportivmassnahmen in der Onkologie (ASO) of the Deutsche Krebsgesellschaft (DKG-German Cancer Society). Ann Hematol $\underline{82}$, $105-$ 117

Ljungberg B, Nilsson-Ehle I (1988): Comparative pharmacokinetics of ceftazidime in young, healthy and elderly, acutely ill males. Eur J Clin Pharmacol 34, 179-186

Mattoes HM, Kuti JL, Drusano GL, Nicolau DP (2004): Optimizing antimicrobial pharmacodynamics: dosage strategies for meropenem. Clin Ther $\underline{26}, 1187-1198$

Moellering RC, Jr., Eliopoulos GM, Sentochnik DE (1989): The carbapenems: new broad spectrum beta-lactam antibiotics. J Antimicrob Chemother 24, 1-7

Moon YS, Chung KC, Gill MA (1997): Pharmacokinetics of meropenem in animals, healthy volunteers, and patients. Clin Infect Dis 24, 249-255

Mouton JW, van den Anker JN (1995): Meropenem clinical pharmacokinetics. Clin Pharmacokinet $28,275-286$

Mouton JW, Vinks AA (1996): Is continuous infusion of beta-lactam antibiotics worthwhile?-efficacy and pharmacokinetic considerations. J Antimicrob Chemother $\underline{38}, 5-15$

Mouton JW, Vinks AA (2005): Relationship between minimum inhibitory concentration and stationary concentration revisited: growth rates and minimum bactericidal concentrations.

Clin Pharmacokinet $\underline{44}, 767-768$ 
Mueller BA, Scarim SK, Macias WL (1993): Comparison of imipenem pharmacokinetics in patients with acute or chronic renal failure treated with continuous hemofiltration. Am $\mathrm{J}$ Kidney Dis $\underline{21}, 172-179$

Nilsson-Ehle I, Hutchison M, Haworth SJ, Norrby SR (1991): Pharmacokinetics of meropenem compared to imipenem-cilastatin in young, healthy males. Eur J Clin Microbiol Infect Dis $\underline{10}, 85-88$

Nyhlen A, Ljungberg B, Nilsson-Ehle I (1997): Pharmacokinetics of meropenem in febrile neutropenic patients. Swedish study group. Eur J Clin Microbiol Infect Dis $\underline{16}$, 797-802

Ong CT, Tessier PR, Li C, Nightingale CH, Nicolau DP (2007): Comparative in vivo efficacy of meropenem, imipenem, and cefepime against Pseudomonas aeruginosa expressing MexAMexB-OprM efflux pumps. Diagn Microbiol Infect Dis 57, 153-161

Pea F, Furlanut M (2001): Pharmacokinetic aspects of treating infections in the intensive care unit: focus on drug interactions. Clin Pharmacokinet $\underline{40}$, 833-868

Pea F, Viale P (2006): The antimicrobial therapy puzzle: could pharmacokineticpharmacodynamic relationships be helpful in addressing the issue of appropriate pneumonia treatment in critically ill patients?. Clin Infect Dis $\underline{42}, 1764-1771$

Pea F, Viale P, Furlanut M (2005a): Antimicrobial therapy in critically ill patients: a review of pathophysiological conditions responsible for altered disposition and pharmacokinetic variability. Clin Pharmacokinet $\underline{44}$, 1009-1034

Pea F, Viale P, Damiani D, Pavan F, Cristini F, Fanin R, Furlanut M (2005b): Ceftazidime in acute myeloid leukemia patients with febrile neutropenia: helpfulness of continuous intravenous infusion in maximizing pharmacodynamic exposure. Antimicrob Agents Chemother $\underline{49}$, 3550-3553

Pfaller MA, Jones RN (1997): A review of the in vitro activity of meropenem and comparative antimicrobial agents tested against 30,254 aerobic and anaerobic pathogens isolated world wide. Diagn Microbiol Infect Dis $\underline{28}, 157-163$

Pinder M, Bellomo R, Lipman J (2002): Pharmacological principles of antibiotic prescription in the critically ill. Anaesth Intensive Care $\underline{30}, 134-144$

Richards MJ, Edwards JR, Culver DH, Gaynes RP (1999): Nosocomial infections in medical intensive care units in the United States. National Nosocomial Infections Surveillance System. Crit Care Med 27, 887-892

Robatel C, Decosterd LA, Biollaz J, Eckert P, Schaller MD, Buclin T (2003): Pharmacokinetics and dosage adaptation of meropenem during continuous venovenous hemodiafiltration in critically ill patients. J Clin Pharmacol 43, 1329-1340

Roberts JA, Kirkpatrick CM, Roberts MS, Robertson TA, Dalley AJ, Lipman J (2009): Meropenem dosing in critically ill patients with sepsis and without renal dysfunction: intermittent bolus versus continuous administration? Monte Carlo dosing simulations and subcutaneous tissue distribution. J Antimicrob Chemother 64, 142-150

Sauermann R, Müller M, Joukhadar C (2005): Penetration von Antibiotika in das schwer erreichbare Kompatiment. Chemother J $\underline{14}$, 74-78 
Schuster H-P, Werdan K: Intensivtherapie bei Sepsis und Multiorganversagen, 3. Auflage, Springer Verlag, Berlin 2000

Sieger B (1998): Meropenem monotherapy as empiric treatment of nosocomial lower respiratory tract infection. Crit Care Med 26, 1462-1463

Simon C, Stille W: Antibiotika-Therapie in Klinik und Praxis, 9. Auflage, Schattauer Verlag, Stuttgart 1997

Tegeder I, Neumann F, Bremer F, Brune K, Lotsch J, Geisslinger G (1999): Pharmacokinetics of meropenem in critically ill patients with acute renal failure undergoing continuous venovenous hemofiltration. Clin Pharmacol Ther $\underline{65}$, 50-57

Thalhammer F, Schenk P, Burgmann H, El M, I, Hollenstein UM, Rosenkranz AR, SunderPlassmann G, Breyer S, Ratheiser K (1998): Single-dose pharmacokinetics of meropenem during continuous venovenous hemofiltration. Antimicrob Agents Chemother $\underline{42}$, 2417-2420

Thalhammer F, Traunmuller F, El M, I, Frass M, Hollenstein UM, Locker GJ, Stoiser B, Staudinger T, Thalhammer-Scherrer R, Burgmann H (1999): Continuous infusion versus intermittent administration of meropenem in critically ill patients. J Antimicrob Chemother $\underline{43}, 523-527$

Thyrum PT, Yeh C, Birmingham B, Lasseter K (1997): Pharmacokinetics of meropenem in patients with liver disease. Clin Infect Dis $\underline{24}, 184-190$

Tomaselli F, Maier A, Matzi V, Smolle-Juttner FM, Dittrich P (2004): Penetration of meropenem into pneumonic human lung tissue as measured by in vivo microdialysis. Antimicrob Agents Chemother $\underline{48}, 2228-2232$

Turner PJ (2000): MYSTIC (Meropenem Yearly Susceptibility Test Information Collection): a global overview. J Antimicrob Chemother $\underline{46}$, 9-23

Turnidge JD (1998): The pharmacodynamics of beta-lactams. Clin Infect Dis $\underline{27}, 10-22$

Valtonen M, Tiula E, Backman JT, Neuvonen PJ (2000): Elimination of meropenem during continuous veno-venous haemofiltration and haemodiafiltration in patients with acute renal failure. J Antimicrob Chemother $\underline{45}$, 701-704

van den Anker JN, Drusano GL, Sorgel F: Dosage recommendations for meropenem in newborn infants. In: 20th International Congress of Chemotherapy, 20th International Congress of Chemotherapy, Sydney 1997, 210-211

Ververs TF, van Dijk A, Vinks SA, Blankestijn PJ, Savelkoul JF, Meulenbelt J, Boereboom FT (2000): Pharmacokinetics and dosing regimen of meropenem in critically ill patients receiving continuous venovenous hemofiltration. Crit Care Med 28, 3412-3416

Vincent JL, Bihari DJ, Suter PM, Bruining HA, White J, Nicolas-Chanoin MH, Wolff M, Spencer RC, Hemmer M (1995): The prevalence of nosocomial infection in intensive care units in Europe. Results of the European Prevalence of Infection in Intensive Care (EPIC) Study. EPIC International Advisory Committee. JAMA 274, 639-644

Vos MC, Vincent HH, Yzerman EP (1992): Clearance of imipenem/cilastatin in acute renal failure patients treated by continuous hemodiafiltration (CAVHD). Intensive Care Med $\underline{18}$, 282-285 
Wise R, Logan M, Cooper M, Ashby JP, Andrews JM (1990): Meropenem pharmacokinetics and penetration into an inflammatory exudate. Antimicrob Agents Chemother $\underline{34}, 1515-1517$

Wiseman LR, Wagstaff AJ, Brogden RN, Bryson HM (1995): Meropenem. A review of its antibacterial activity, pharmacokinetic properties and clinical efficacy. Drugs $\underline{50}, 73-101$ 


\section{$7 \quad$ Abkürzungsverzeichnis}

$\mathrm{AF}$

ANV

AUC

BAL

BK

$\mathrm{Cl}$

$\mathrm{Cl}_{\text {krea }}$

$\mathrm{Cl}_{\text {mer }}$

$\mathrm{C}_{\max }$

$\mathrm{C}_{\min }$

CVVHF

FUO

$\mathrm{HF}$

IBW

$\mathrm{k}_{\mathrm{el}}$

Krea

MIC

MRS

MRT

NP

Nicht-NP

PAE

SIRS

$\mathrm{T}_{1 / 2 \alpha}$

$\mathrm{T}_{1 / 2 \beta}$

$\mathrm{V}_{\mathrm{d}}$

$\mathrm{V}_{\mathrm{ss}}$
Atemfrequenz

akutes Nierenversagen

Area under the curve (Fläche unter der Zeit-Konzentrations-Kurve)

Bronchoalveoläre Lavage

Blutkultur

Clearance

Kreatinin-Clearance

Meropenem-Clearance

Maximumkonzentration

Minimumkonzentration

kontinuierliche veno-venöse Hämofiltration

Fever of unknown origin

Herzfrequenz

ideal body weight

Eliminationskonstante

Kreatinin

minimal inhibitory concentration (minimale Hemmkonzentration)

Methicillin-resistente Staphylokokken

Mean residence time

neutropenische Patienten

nicht-neutropenische Patienten

postantibiotischer Effekt

systemic inflammatory response syndrome

Halbwertszeit während der Verteilungsphase

Halbwertszeit während der Eliminationsphase

Verteilungsvolumen

Verteilungsvolumen im Steady state 


\begin{tabular}{|c|c|c|c|c|c|c|}
\hline Pat.Nr. & Alter & Geschlecht & $\begin{array}{l}\text { Größe } \\
(\mathrm{cm})\end{array}$ & $\begin{array}{l}\text { Gewicht } \\
\quad(\mathrm{kg})\end{array}$ & Diagnose & weitere relevante Erkrankungen \\
\hline 1 & 47 & $\mathrm{~W}$ & 160 & 65 & $\begin{array}{l}\text { niedrigmalignes T-Zell Non-Hodgkin-Lymphom; } \\
\text { Z.n. Hoch Dosis-Chemotherapie und } \\
\text { Stammzelltransplantation }\end{array}$ & Progression d. T-Zell-Lymphom, FUO \\
\hline 2 & 60 & $\mathrm{~W}$ & 165 & 65 & $\begin{array}{l}\text { AML (M4 nach FAB), akute Panzytopenie, } \\
\text { Z.n. Induktionschemotherapie nach HAM- } \\
\text { Schema }\end{array}$ & V.a. Pyelonephritis, Schüttelfrost, Rhinitis \\
\hline 3 & 40 & $\mathrm{~W}$ & 165 & 79 & $\begin{array}{l}\text { hochmalignes B-Non-Hodgkin-Lymphom, } \\
\text { Blastenschub, Z.n.1.Zyklus CHOEP und } \\
\text { 1.Zyklus DHAP }\end{array}$ & $\begin{array}{l}\text { Lymphome li. Axilla, supravesical u. intestinal } \\
\text { (Z.n. Dünndarmileus und gastrointestinale Ulcera) }\end{array}$ \\
\hline 4 & 60 & M & 166 & 67,7 & $\begin{array}{l}\text { IgG-Plasmozytom mit Bence-Jones-Proteinurie, } \\
\text { Z.n. HO-Chemotherapie }\end{array}$ & FUO, Z.n. rezidivierenden Pneumonien \\
\hline 5 & 75 & M & 172 & 75 & AML im Anfangsstadium & $\begin{array}{l}\text { Panzytopenie, Anämie, Leukozytopenie, art.Hypertonus, } \\
\text { Thrombophlebitis li Ellenbeuge }\end{array}$ \\
\hline 6 & 47 & M & 175 & 75 & Z.n. T-ALL, Z.n. KM-Transplantation (allogen) & HWI, V.a. CMV-Reaktivierung \\
\hline 7 & 57 & M & 170 & 80,2 & $\begin{array}{l}\text { AML (M4), } \\
\text { Z.n. Induktionschemotherapie }\end{array}$ & Bakteriämie, art. Hypertonus \\
\hline 8 & 53 & M & 175 & 85,1 & sek. AML & Bakteriämie, Panzytopenie \\
\hline 9 & 63 & $\mathrm{~W}$ & 170 & 75,3 & AML M5, Z.n. Polychemotherapie & $\begin{array}{l}\text { Pneumonie, inkomplette Blastenreduktion, } \\
\text { Blutungsneigung }\end{array}$ \\
\hline 10 & 35 & $\mathrm{~W}$ & 161 & 48 & $\begin{array}{l}\text { M.Hodgkin Rezidiv, } \\
\text { Therapiefortsetzung BEAD }\end{array}$ & Pneumonie \\
\hline $\begin{array}{l}\text { Median } \\
\text { SD }\end{array}$ & $\begin{aligned} & 55 \\
\pm & 11,8\end{aligned}$ & $\begin{array}{l}5 \mathrm{M} \\
5 \mathrm{~W}\end{array}$ & $\begin{array}{c}168 \\
\pm 5,3\end{array}$ & $\begin{aligned} & 75 \\
\pm & 10,6\end{aligned}$ & & \\
\hline
\end{tabular}

Tabelle 7: Diagnosen und weitere relevante Erkrankungen der hämatoonkologischen Patienten 


\begin{tabular}{|c|c|c|c|c|c|c|c|c|c|c|c|c|c|c|c|c|c|c|c|c|}
\hline \multirow[t]{2}{*}{ Pat.Nr. } & \multicolumn{3}{|c|}{ Elektrolyte } & \multirow{2}{*}{$\begin{array}{l}\text { Niere } \\
\mathrm{Krea} \\
{[\mathrm{mg} / \mathrm{dl}]}\end{array}$} & \multicolumn{2}{|c|}{ Protein } & \multicolumn{5}{|c|}{ Leber/Galle } & \multicolumn{4}{|c|}{ Gerinnung } & \multicolumn{5}{|c|}{ Blutbild } \\
\hline & $\mid \begin{array}{c}\mathrm{Na} \\
{[\mathrm{mmol} / \mathrm{l}]}\end{array}$ & $\begin{array}{c}K \\
{[\mathrm{mmol} / \mathrm{ll}]}\end{array}$ & $\begin{array}{c}\mathrm{Ca} \\
{[\mathrm{mmol} / \mathrm{l}]}\end{array}$ & & Prot & {$\left[\begin{array}{l}A l b \\
{[g / d l]}\end{array}\right.$} & $\begin{array}{l}A S T \\
{[U / l]}\end{array}$ & $\begin{array}{l}A L T \\
{[U / l]}\end{array}$ & $\begin{array}{l}y-G T \\
{[U / l]}\end{array}$ & {$\left[\begin{array}{c}A P \\
{[U / l]}\end{array}\right.$} & {$\left[\begin{array}{c}B i l i \\
{[m g / d l]}\end{array}\right]$} & $\begin{array}{l}\text { Quick } \\
{[\%]}\end{array}$ & $\begin{array}{l}a P T T \\
{[\text { sek] }}\end{array}$ & $\begin{array}{l}\text { Fibr } \\
{[m g / d l]}\end{array}$ & $\begin{array}{c}A T I I I \\
{[\%]}\end{array}$ & $\begin{array}{l}H b \\
{[g / d l]}\end{array}$ & $\begin{array}{l}H k \\
{[\%]}\end{array}$ & $\begin{array}{c}\text { Erys } \\
{\left[x 10^{6} / \mu l\right]}\end{array}$ & $\begin{array}{c}\text { Leukos } \\
{\left[x 10^{3} / \mu l\right]}\end{array}$ & $\begin{array}{l}\text { Thrombos } \\
{\left[\times 10^{3} / \mu l\right]}\end{array}$ \\
\hline $\begin{array}{l}\text { Referenz- } \\
\text { bereich }\end{array}$ & $136-145$ & $3,5-4,8$ & $2,2-2,65$ & $\begin{array}{ll}M & 0,72-1,18 \\
W & 0,55-1,02\end{array}$ & $8,6-$ & $\begin{array}{c}3,4- \\
4,8\end{array}$ & $<=15$ & $<=17$ & $<=19$ & $\begin{array}{l}60- \\
180\end{array}$ & $<=1,2$ & $70-130$ & $29-40$ & $170-400$ & $80-130$ & $11,5-15$ & $35-46$ & $3,9-5,1$ & $4,0-11,0$ & $150-300$ \\
\hline 1 & 137 & $3,4^{*}$ & 2,4 & $0,5^{*}$ & 7,1 & 3,7 & 15 & 15 & $142 *$ & $354 *$ & $1,8^{*}$ & 114 & 37 & $694 *$ & 88 & $10,0^{*}$ & $27,9 *$ & $3,4^{*}$ & $0,11^{*}$ & $15^{*}$ \\
\hline 2 & $132 *$ & $3,0^{*}$ & $1,9 *$ & 0,9 & $5,6^{*}$ & $2,8^{*}$ & $28 *$ & $51^{*}$ & $69^{*}$ & $203 *$ & 0,3 & 99 & $42 *$ & $437^{*}$ & $77^{*}$ & $9,7^{*}$ & $26,7^{*}$ & $3,4^{*}$ & $1,83^{*}$ & $17^{*}$ \\
\hline 3 & $131 *$ & 4,3 & $3,0^{*}$ & 0,6 & $5,7 *$ & $2,8^{*}$ & $25 *$ & 6 & 13 & 158 & 0,9 & 90 & 33 & $626^{*}$ & - & $8,3^{*}$ & $22,2 *$ & $2,8^{*}$ & $13,73 *$ & $316^{*}$ \\
\hline 4 & 141 & $3,3^{*}$ & $2,1^{*}$ & 1,1 & 6,8 & 3,4 & 10 & 5 & 11 & 126 & 0,5 & - & - & - & - & $10,1^{*}$ & $29,6^{*}$ & $3,8^{*}$ & 5,87 & $40 *$ \\
\hline 5 & 139 & 4,4 & 2,2 & 1,1 & 6,7 & 3,4 & 10 & 17 & 10 & 98 & 0,4 & 112 & 30 & $619 *$ & 91 & $9,0^{*}$ & $24,8^{*}$ & $2,8^{*}$ & $1,62 *$ & $64^{*}$ \\
\hline 6 & 137 & 4,4 & 2,2 & $1,5^{*}$ & $5,6^{*}$ & $3,0^{*}$ & $124 *$ & $217^{*}$ & $168^{*}$ & $347 *$ & 1 & 106 & 35 & $385^{*}$ & - & $10,7^{*}$ & $31,3 *$ & $3,6^{*}$ & $2,40^{*}$ & $76^{*}$ \\
\hline 7 & 139 & 3,9 & 2,3 & 1,1 & 7,0 & 3,4 & 10 & $31^{*}$ & 19 & 87 & 1 & 101 & 32 & $600 *$ & 90 & $8,8^{*}$ & $23,9^{*}$ & $2,9 *$ & $0,05^{*}$ & $29 *$ \\
\hline 8 & $135^{*}$ & 4,3 & 2,3 & 1,0 & 7,4 & $2,8^{*}$ & 6 & 9 & $30 *$ & 89 & $1,6^{*}$ & 95 & 35 & $688^{*}$ & $76^{*}$ & $9,2^{*}$ & $26,1^{*}$ & $2,7^{*}$ & $0,14^{*}$ & $13 *$ \\
\hline 9 & 140 & $3,4^{*}$ & $2,1^{*}$ & 0,8 & $6,2 *$ & 3,1 & & 4 & 15 & 89 & $1,3^{*}$ & - & - & - & - & $8,7^{*}$ & $25,3^{*}$ & $3,0^{*}$ & $0,30 *$ & $26^{*}$ \\
\hline 10 & - & $3,3^{*}$ & 2,4 & $0,5^{*}$ & 6,9 & 3,8 & 10 & 9 & - & $123 *$ & 1,2 & 103 & 37 & $976^{*}$ & 85 & $10,0^{*}$ & $29,1 *$ & $3,3^{*}$ & $0,13^{*}$ & $15^{*}$ \\
\hline
\end{tabular}

* Werte außerhalb des Referenzbereichs

Tabelle 8: Laborparameter der hämatoonkologischen Patienten 


\begin{tabular}{|c|c|c|c|c|c|c|c|c|c|c|}
\hline PatNr. & $\begin{array}{l}\text { Infekt- } \\
\text { nachweis }\end{array}$ & $\begin{array}{c}\text { Fieberursache/ } \\
\text { Rö-Thorax-Befund }\end{array}$ & Mikrobiologie & $\left.\begin{array}{l}\text { Leukos } \\
{[\mathrm{Tsd} / \mu \mathrm{l}]}\end{array}\right]$ & $\begin{array}{l}\text { Neutro- } \\
\text { penie }\end{array}$ & $\begin{array}{c}\mathrm{CRP} \\
{[\mathrm{mg} / \mathrm{l}]}\end{array}$ & $\begin{array}{l}\text { Temp } \\
{\left[{ }^{\circ} \mathrm{C}\right]}\end{array}$ & $\begin{array}{c}\mathrm{HF} \\
{\left[\mathrm{min}^{-1}\right]}\end{array}$ & $\left.\begin{array}{l}\text { RRsyst } \\
{[\mathrm{mmHg}]}\end{array}\right]$ & $\begin{array}{l}\text { Schwere- } \\
\text { grad der } \\
\text { Infektion* }\end{array}$ \\
\hline 1 & FUO & --- & --- & 0,11 & $\mathrm{Ja}$ & 78,0 & 38,4 & 120 & 150 & $\begin{array}{c}\text { wahrscheinliche } \\
\text { Sepsis }\end{array}$ \\
\hline 2 & CMDI & HWI, akute Nephritis & Mittelstrahlurin: Enterobacter spec. & 1,83 & Nein & 100,5 & 38,4 & 108 & 137 & $\begin{array}{l}\text { wahrscheinliche } \\
\text { Sepsis }\end{array}$ \\
\hline 3 & FUO & B-Symptomatik bei Blastenschub & -- & 13,73 & $\mathrm{Ja}$ & 175,0 & 38,6 & 132 & 115 & $\begin{array}{l}\text { wahrscheinliche } \\
\text { Sepsis }\end{array}$ \\
\hline 4 & FUO & --- & -- & 5,87 & Nein & 55,2 & 38,9 & 66 & --- & keine Sepsis \\
\hline 5 & $\mathrm{CDI}$ & Thrombophlebitis & -- & 1,62 & Nein & 43,7 & 35,6 & 80 & --- & keine Sepsis \\
\hline 6 & CMDI & HWI & Urin: E.faecalis, Staph.epidermidis & 2,40 & Nein & 46,1 & 38,2 & 96 & 105 & keine Sepsis \\
\hline 7 & CMDI & Bakteriämie mit unklaren Focus & Blutkultur: Enterobacter cloacae & 0,05 & $\mathrm{Ja}$ & 100,0 & 39,9 & 92 & 140 & Sepsis \\
\hline 8 & CMDI & Bakteriämie mit unklaren Focus & Blutkultur: Staph.epidermidis & 0,14 & $\mathrm{Ja}$ & 260,0 & 38,5 & 110 & 108 & Sepsis \\
\hline 9 & CMDI & Pneumonie & Bronchialsekret: koag.neg.Staph. & 0,30 & $\mathrm{Ja}$ & 110,9 & 38,0 & 104 & 135 & $\begin{array}{l}\text { wahrscheinliche } \\
\text { Sepsis }\end{array}$ \\
\hline 10 & CDI & Pneumonie & Blutkultur: koag. neg. Staphylokokken & 0,13 & $\mathrm{Ja}$ & 285,9 & 37,2 & 80 & 107 & Sepsis \\
\hline
\end{tabular}

* Kriterien siehe Text (Seite 30)

Tabelle 9: Infektionsparameter der hämatoonkologischen Patienten 


\begin{tabular}{|c|c|c|c|c|c|c|c|c|c|c|c|}
\hline Pat.Nr. & Geschlecht & $\begin{array}{l}\text { Gewicht } \\
\quad(\mathrm{kg})\end{array}$ & $\begin{array}{c}\text { Größe } \\
(\mathrm{cm})\end{array}$ & $\begin{array}{c}\text { Alter } \\
\text { (Jahre) }\end{array}$ & $\begin{array}{c}\text { Krea } \\
(\mathrm{mg} / \mathrm{dl})\end{array}$ & $\begin{array}{l}\text { IBW } \\
(\mathrm{kg})\end{array}$ & $\begin{array}{c}\mathrm{Cl}_{\text {krea }} \\
(\mathrm{ml} / \mathrm{min})\end{array}$ & $\begin{array}{r}\text { Dosis } \\
(\mathrm{mg})\end{array}$ & $\begin{array}{l}\text { Intervall- } \\
\text { dauer (h) }\end{array}$ & $\begin{array}{l}\text { Infusions- } \\
\text { intervall (n) }\end{array}$ & $\begin{array}{l}\text { Infusions- } \\
\text { dauer (h) }\end{array}$ \\
\hline 1 & $\mathrm{~W}$ & 65,0 & 160 & 47 & 0,5 & 57,7 & 126,7 & 1000 & 8 & 1 & 0,47 \\
\hline 2 & W & 65,0 & 165 & 60 & 0,7 & 62,4 & 84,2 & 1000 & 8 & 7 & 0,48 \\
\hline 3 & w & 77,9 & 165 & 40 & 0,6 & 62,4 & 122,8 & 1000 & 8 & 2 & 0,53 \\
\hline 4 & $\mathrm{~m}$ & 67,7 & 166 & 60 & 1,0 & 62,3 & 69,2 & 1000 & 8 & 4 & 0,30 \\
\hline 5 & $\mathrm{~m}$ & 75,0 & 172 & 75 & 1,1 & 67,7 & 55,6 & 1000 & 8 & 3 & 0,20 \\
\hline 6 & $\mathrm{~m}$ & 75,0 & 175 & 47 & 1,4 & 70,5 & 65,0 & 1000 & 8 & 2 & 0,17 \\
\hline 7 & $\mathrm{~m}$ & 80,2 & 170 & 57 & 1,0 & 65,9 & 76,0 & 1000 & 8 & 2 & 0,45 \\
\hline 8 & $\mathrm{~m}$ & 85,1 & 175 & 53 & 0,9 & 70,5 & 94,6 & 1000 & 8 & 2 & 0,20 \\
\hline 9 & w & 75,3 & 170 & 63 & 0,8 & 67,1 & 76,2 & 1000 & 8 & 3 & 0,42 \\
\hline 10 & $\mathrm{w}$ & 48,0 & 161 & 35 & 0,5 & 58,6 & 120,3 & 1000 & 8 & 6 & 0,32 \\
\hline Mittelwert & & 71,4 & 168 & 54 & 0,9 & 64,5 & 89,1 & 1000 & 8 & 3,2 & 0,35 \\
\hline Median & & 75,0 & 168 & 55 & 0,9 & 64,2 & 80,2 & 1000 & 8 & 2,5 & 0,37 \\
\hline SD & & 10,5 & 5 & 12 & 0,3 & 4,5 & 25,9 & 0 & 0 & 1,9 & 0,13 \\
\hline Min & & 48,0 & 160 & 35 & 0,5 & 57,7 & 55,6 & 1000 & 8 & 1,0 & 0,17 \\
\hline Max & & 85,1 & 175 & 75 & 1,4 & 70,5 & 126,7 & 1000 & 8 & 7,0 & 0,53 \\
\hline
\end{tabular}

Tabelle 10: Pharmakokinetische Parameter der hämatoonkologischen Patienten Teil 1 


\begin{tabular}{|c|c|c|c|c|c|c|c|c|c|c|c|c|c|c|}
\hline Pat.Nr. & $\begin{array}{l}\mathrm{Cmax} \\
(\mathrm{mg} / \mathrm{l})\end{array}$ & $\begin{array}{l}\mathrm{Cmin} \\
(\mathrm{mg} / \mathrm{l})\end{array}$ & $\begin{array}{c}\mathrm{Cl}_{\text {mer }} \\
(\mathrm{ml} / \mathrm{min})\end{array}$ & $\begin{array}{l}\mathrm{V}_{\mathrm{ss}} \\
\text { (l) }\end{array}$ & $\begin{array}{c}\mathrm{V}_{\mathrm{ss}} \\
(1 / \mathrm{kg})\end{array}$ & $\begin{array}{l}\text { MRT } \\
(\min )\end{array}$ & $\begin{array}{c}\mathrm{k}_{\mathrm{el}} \\
(1 / \mathrm{h})\end{array}$ & $\begin{array}{c}T_{1 / 2 \alpha} \\
(\mathrm{h})\end{array}$ & $\begin{array}{c}T_{1 / 2 \beta} \\
(\mathrm{h})\end{array}$ & $\begin{array}{c}\operatorname{AUC}(0-\infty) \\
(\mathrm{mg} * \mathrm{~h} / \mathrm{l})\end{array}$ & $\begin{array}{c}\mathrm{T}>2 \mathrm{mg} / \mathrm{l} \\
\text { (h) }\end{array}$ & $\begin{array}{c}\mathrm{T}>4 \mathrm{mg} / \mathrm{l} \\
\text { (h) }\end{array}$ & $\begin{array}{c}\mathrm{T}>2 \mathrm{mg} / \mathrm{l} \\
(\%)\end{array}$ & $\begin{array}{c}\mathrm{T}>4 \mathrm{mg} / \mathrm{l} \\
(\%)\end{array}$ \\
\hline 1 & 52,93 & 0,00 & 345,34 & 14,95 & 0,23 & 43,3 & 1,39 & 0,34 & 0,75 & 48,26 & 2,79 & 2,19 & 34,82 & 27,42 \\
\hline 2 & 48,30 & 2,82 & 169,41 & 34,54 & 0,53 & 203,4 & 0,29 & 0,37 & 3,10 & 98,38 & 9,33 & 6,73 & 100,00 & 84,12 \\
\hline 3 & 38,22 & 0,44 & 290,6 & 27,32 & 0,35 & 94,0 & 0,638 & 0,39 & 1,43 & 57,34 & 5,26 & 3,77 & 65,81 & 47,16 \\
\hline 4 & 69,26 & 1,55 & 158,71 & 21,12 & 0,31 & 132,3 & 0,45 & 0,62 & 2,89 & 105,02 & 7,91 & 4,17 & 98,90 & 52,14 \\
\hline 5 & 52,44 & 1,80 & 178,36 & 37,0 & 0,49 & 206,0 & 0,29 & 0,74 & 4,77 & 93,44 & 4,65 & 3,73 & 58,11 & 46,59 \\
\hline 6 & 47,77 & 2,83 & 138,55 & 24,45 & 0,33 & 178,6 & 0,34 & 0,33 & 2,14 & 120,30 & 9,43 & 7,14 & 100,00 & 89,25 \\
\hline 7 & 38,46 & 0,48 & 254,22 & 25,31 & 0,32 & 99,6 & 0,60 & 0,57 & 1,38 & 65,56 & 5,60 & 4,31 & 70,06 & 53,92 \\
\hline 8 & 69,90 & 0,08 & 251,93 & 15,30 & 0,18 & 60,8 & 0,99 & 0,36 & 0,93 & 66,16 & 6,56 & 4,45 & 81,95 & 55,67 \\
\hline 9 & 72,73 & 0,66 & 174,44 & 16,19 & 0,22 & 92,8 & 0,65 & 0,23 & 1,34 & 95,54 & 6,29 & 4,82 & 78,59 & 60,24 \\
\hline 10 & 64,93 & 0,21 & 215,47 & 16,54 & 0,34 & 76,9 & 0,78 & 0,21 & 1,01 & 77,35 & 5,03 & 4,03 & 62,90 & 50,32 \\
\hline Mittelwert & 55,49 & 1,09 & 217,70 & 23,27 & 0,33 & 118,77 & 0,64 & 0,42 & 1,97 & 82,74 & 6,29 & 4,53 & 75,11 & 56,68 \\
\hline Median & 52,69 & 0,57 & 196,92 & 22,79 & 0,33 & 96,80 & 0,62 & 0,37 & 1,41 & 85,40 & 5,95 & 4,24 & 74,33 & 53,03 \\
\hline SD & 12,91 & 1,09 & 66,34 & 7,96 & 0,11 & 58,69 & 0,35 & 0,17 & 1,27 & 23,25 & 2,10 & 1,45 & 21,18 & 18,01 \\
\hline Min & 38,22 & 0,00 & 138,55 & 14,95 & 0,18 & 43,30 & 0,29 & 0,21 & 0,75 & 48,26 & 2,79 & 2,19 & 34,82 & 27,42 \\
\hline $\operatorname{Max}$ & 72,73 & 2,83 & 345,34 & 37,00 & 0,53 & 206,00 & 1,39 & 0,74 & 4,77 & 120,30 & 9,43 & 7,14 & 100,00 & 89,25 \\
\hline
\end{tabular}

Tabelle 11: Pharmakokinetische Parameter der hämatoonkologischen Patienten Teil 2 


\begin{tabular}{|c|c|c|c|c|c|c|c|c|c|c|c|}
\hline Pat.Nr. & Geschlecht & $\begin{array}{l}\text { Gewicht } \\
(\mathrm{kg})\end{array}$ & $\begin{array}{c}\text { Größe } \\
(\mathrm{cm})\end{array}$ & $\begin{array}{c}\text { Alter } \\
\text { (Jahre) }\end{array}$ & $\begin{array}{c}\text { Krea } \\
(\mathrm{mg} / \mathrm{dl})\end{array}$ & $\begin{array}{l}\text { IBW } \\
(\mathrm{kg})\end{array}$ & $\begin{array}{c}\mathrm{Cl}_{\text {krea }} \\
(\mathrm{ml} / \mathrm{min})\end{array}$ & $\begin{array}{l}\text { Dosis } \\
(\mathrm{mg})\end{array}$ & $\begin{array}{l}\text { Intervall- } \\
\text { dauer (h) }\end{array}$ & $\begin{array}{l}\text { Infusions- } \\
\text { Intervall (n) }\end{array}$ & $\begin{array}{c}\text { Infusions- } \\
\text { dauer (h) }\end{array}$ \\
\hline \multicolumn{12}{|c|}{ Nicht-Neutropeniker } \\
\hline 2 & $\mathrm{~W}$ & 65,0 & 165 & 60 & 0,7 & 62,4 & 84,2 & 1000 & 8 & 7 & 0,48 \\
\hline 4 & M & 67,7 & 166 & 60 & 1,0 & 62,3 & 69,2 & 1000 & 8 & 4 & 0,30 \\
\hline 5 & M & 75,0 & 172 & 75 & 1,1 & 67,7 & 55,6 & 1000 & 8 & 3 & 0,20 \\
\hline 6 & M & 75,0 & 175 & 47 & 1,4 & 70,5 & 65,0 & 1000 & 8 & 2 & 0,17 \\
\hline Mittelwert & & 70,68 & 169,5 & 60,5 & 1,05 & 65,7 & 68,50 & 1000 & 8 & 4 & 0,29 \\
\hline Median & & 71,35 & 169 & 60 & 1,05 & 65,1 & 67,10 & 1000 & 8 & 3,50 & 0,25 \\
\hline $\mathrm{SD}$ & & 5,11 & 4,80 & 11,45 & 0,29 & 4,06 & 11,91 & 0 & 0 & 2,16 & 0,14 \\
\hline Min & & 65 & 165 & 47 & 0,7 & 62,3 & 55,60 & 1000 & 8 & 2 & 0,17 \\
\hline Max & & 75 & 175 & 75 & 1,4 & 70,5 & 84,20 & 1000 & 8 & 7 & 0,48 \\
\hline \multicolumn{12}{|c|}{ Neutropeniker } \\
\hline 1 & W & 65,0 & 160 & 47 & 0,5 & 57,7 & 126,7 & 1000 & 8 & 1 & 0,47 \\
\hline 3 & W & 77,9 & 165 & 40 & 0,6 & 62,4 & 122,8 & 1000 & 8 & 2 & 0,53 \\
\hline 7 & M & 80,2 & 170 & 57 & 1,0 & 65,9 & 76,0 & 1000 & 8 & 2 & 0,45 \\
\hline 8 & M & 85,1 & 175 & 53 & 0,9 & 70,5 & 94,6 & 1000 & 8 & 2 & 0,20 \\
\hline 9 & W & 75,3 & 170 & 63 & 0,8 & 67,1 & 76,2 & 1000 & 8 & 3 & 0,42 \\
\hline 10 & W & 48,0 & 161 & 35 & 0,5 & 58,6 & 120,3 & 1000 & 8 & 6 & 0,32 \\
\hline Mittelwert & & 72 & 167 & 49 & 0,72 & 63,70 & 102,77 & 1000 & 8 & 2,67 & 0,40 \\
\hline Median & & 76,6 & 167,5 & 50 & 0,70 & 64,15 & 107,45 & 1000 & 8 & 2 & 0,44 \\
\hline $\mathrm{SD}$ & & 13,49 & 5,85 & 10,55 & 0,21 & 5,03 & 23,54 & 0 & 0 & 1,75 & 0,12 \\
\hline Min & & 48 & 160 & 35 & 0,5 & 57,7 & 76,0 & 1000 & 8 & 1 & 0,20 \\
\hline Max & & 85 & 175 & 63 & 1,0 & 70,5 & 126,7 & 1000 & 8 & 6 & 0,53 \\
\hline $\mathrm{P}$ & & 0,867 & 0,472 & 0,458 & 0,068 & 0,522 & $\begin{array}{ll}0,029 \\
\end{array}$ & - & - & 0,312 & 0,245 \\
\hline
\end{tabular}

Tabelle 12: Pharmakokinetische Parameter der hämatoonkologischen Patienten unterteilt in Neutropeniker und Nicht-Neutropeniker Teil 1 


\begin{tabular}{|c|c|c|c|c|c|c|c|c|c|c|c|c|}
\hline Pat.Nr. & $\begin{array}{c}\mathrm{C}_{\max } \\
(\mathrm{mg} / \mathrm{l})\end{array}$ & $\begin{array}{c}\mathrm{C}_{\min } \\
(\mathrm{mg} / \mathrm{l})\end{array}$ & $\begin{array}{c}\mathrm{Cl} \\
(\mathrm{ml} / \mathrm{min})\end{array}$ & $\begin{array}{l}V_{\mathrm{ss}} \\
\text { (1) }\end{array}$ & $\begin{array}{c}\mathrm{V}_{\mathrm{ss}} \\
(\mathrm{l} / \mathrm{kg})\end{array}$ & $\begin{array}{l}\mathrm{T}_{1 / 2 \alpha} \\
\text { (h) }\end{array}$ & $\begin{array}{l}\mathrm{T}_{1 / 2 \beta} \\
(\mathrm{h})\end{array}$ & $\begin{array}{l}\mathrm{AUC}_{0-\infty} \\
\left(\mathrm{mg}{ }^{*} \mathrm{~h} / \mathrm{l}\right)\end{array}$ & $\begin{array}{c}\mathrm{T}>2 \mathrm{mg} / \mathrm{l} \\
(\mathrm{h})\end{array}$ & $\begin{array}{c}\mathrm{T}>4 \mathrm{mg} / 1 \\
\quad(\mathrm{~h})\end{array}$ & $\begin{array}{c}\mathrm{T}>2 \mathrm{mg} / \mathrm{l} \\
(\%)\end{array}$ & $\begin{array}{c}\mathrm{T}>4 \mathrm{mg} / 1 \\
(\%)\end{array}$ \\
\hline \multicolumn{13}{|c|}{ Nicht-Neutropeniker } \\
\hline 2 & 48,3 & 2,82 & 169,41 & 34,54 & 0,53 & 0,37 & 3,1 & 98,38 & 9,33 & 6,73 & 100 & 84,12 \\
\hline 4 & 69,26 & 1,55 & 158,71 & 21,12 & 0,31 & 0,62 & 2,89 & 105,02 & 7,91 & 4,17 & 98,9 & 52,14 \\
\hline 5 & 52,44 & 1,8 & 178,36 & 37 & 0,49 & 0,74 & 4,77 & 93,44 & 4,65 & 3,73 & 58,11 & 46,59 \\
\hline 6 & 47,77 & 2,83 & 138,55 & 24,45 & 0,33 & 0,33 & 2,14 & 120,3 & 9,43 & 7,14 & 100 & 89,25 \\
\hline Mittelwert & 54,44 & 2,25 & 161,26 & 29,28 & 0,42 & 0,52 & 3,23 & 104,29 & 7,83 & 5,44 & 89,25 & 68,03 \\
\hline Median & 50,37 & 2,31 & 164,06 & 29,50 & 0,41 & 0,50 & 3,00 & 101,70 & 8,62 & 5,45 & 99,45 & 68,13 \\
\hline SD & 10,10 & 0,67 & 17,14 & 7,69 & 0,11 & 0,20 & 1,11 & 11,68 & 2,23 & 1,74 & 20,77 & 21,77 \\
\hline Min & 47,77 & 1,55 & 138,55 & 21,12 & 0,31 & 0,33 & 2,14 & 93,44 & 4,65 & 3,73 & 58,11 & 46,59 \\
\hline Max & 69,26 & 2,83 & 178,36 & 37,00 & 0,53 & 0,74 & 4,77 & 120,30 & 9,43 & 7,14 & 100,00 & 89,25 \\
\hline \multicolumn{13}{|c|}{ Neutropeniker } \\
\hline 1 & 52,93 & 0 & 345,34 & 14,95 & 0,23 & 0,34 & 0,75 & 48,26 & 2,79 & 2,19 & 34,82 & 27,42 \\
\hline 3 & 38,22 & 0,44 & 290,6 & 27,32 & 0,35 & 0,39 & 1,43 & 57,34 & 5,26 & 3,77 & 65,81 & 47,16 \\
\hline 7 & 38,46 & 0,48 & 254,22 & 25,31 & 0,32 & 0,57 & 1,38 & 65,56 & 5,6 & 4,31 & 70,06 & 53,92 \\
\hline 8 & 69,9 & 0,08 & 251,93 & 15,3 & 0,18 & 0,36 & 0,93 & 66,16 & 6,56 & 4,45 & 81,95 & 55,67 \\
\hline 9 & 72,73 & 0,66 & 174,44 & 16,19 & 0,22 & 0,23 & 1,34 & 95,54 & 6,29 & 4,82 & 78,59 & 60,24 \\
\hline 10 & 64,93 & 0,21 & 215,47 & 16,54 & 0,34 & 0,21 & 1,01 & 77,35 & 5,03 & 4,03 & 62,9 & 50,32 \\
\hline Mittelwert & 56,20 & 0,31 & 255,33 & 19,27 & 0,27 & 0,35 & 1,14 & 68,37 & 5,26 & 3,93 & 65,69 & 49,12 \\
\hline Median & 58,93 & 0,33 & 253,08 & 16,37 & 0,28 & 0,35 & 1,18 & 65,86 & 5,43 & 4,17 & 67,94 & 52,12 \\
\hline $\mathrm{SD}$ & 15,40 & 0,26 & 59,14 & 5,53 & 0,07 & 0,13 & 0,28 & 16,48 & 1,34 & 0,92 & 16,80 & 11,54 \\
\hline Min & 38,22 & 0,00 & 174,44 & 14,95 & 0,18 & 0,21 & 0,75 & 48,26 & 2,79 & 2,19 & 34,82 & 27,42 \\
\hline Max & 72,73 & 0,66 & 345,34 & 27,32 & 0,35 & 0,57 & 1,43 & 95,54 & 6,56 & 4,82 & 81,95 & 60,24 \\
\hline $\mathrm{p}$ & 0,8474 & 0,0002 & 0,0160 & 0,0422 & 0,0386 & 0,1456 & 0,0020 & 0,0057 & 0,0500 & 0,1071 & 0,0824 & 0,1074 \\
\hline
\end{tabular}

Tabelle 13: Pharmakokinetische Parameter der hämatoonkologischen Patienten unterteilt in Neutropeniker und Nicht-Neutropeniker Teil 2 


\begin{tabular}{|c|c|c|c|c|c|c|}
\hline Pat.Nr. & $\begin{array}{c}\text { Alter } \\
\text { (Jahre) }\end{array}$ & Geschlecht & $\begin{array}{l}\text { Größe } \\
(\mathrm{cm})\end{array}$ & $\begin{array}{l}\text { Gewicht } \\
(\mathrm{kg})\end{array}$ & Diagnose & weitere relevante Erkrankungen \\
\hline 11 & 51 & M & 182 & 128,5 & $\begin{array}{l}\text { kardiale Dekompensation mit Lungenödem bei V.a. } \\
\text { dilatative CMP ( nutritiv-tox.), Z.n. VWI, } \\
\text { katecholaminpflich. Kreislaufschock }\end{array}$ & Stauungspneumonie mit Sepsis \\
\hline 12 & 74 & M & 165 & 91 & Coma hepaticum, obere GI-Blutung, Kardia-Ulkus & $\begin{array}{l}\text { Leberinsuff., Ösophagusvarizen Grad 1-2; Verschlussikterus, } \\
\text { Cholecystolithiasis, art.Hypertonus }\end{array}$ \\
\hline 13 & 59 & $\mathrm{~W}$ & 160 & 72 & $\begin{array}{l}\text { kardiogener Schock bei V.a. dilat.CMP; ANV und } \\
\text { Leberfunktionseinschränkung }\end{array}$ & untere GI-Blutung; V.a.Leberzirrhose \\
\hline 14 & 42 & $\mathrm{~W}$ & 172 & 73 & $\begin{array}{l}\text { Toxic Shock bei nekrotisierender Fasciitis Mamma, } \\
\text { beginnende Verbrauchskoagulopathie, Thrombopenie, } \\
\text { Leukopenie, Z.n. Nekrosektomie }\end{array}$ & HIT Typ II \\
\hline 15 & 45 & M & & & $\begin{array}{l}\text { V.a. Leberzirrhose bei C2-Abusus } \\
\text { Ösophagusvarizenblutung bei akuter } \\
\text { Pfortaderthrombose }\end{array}$ & $\begin{array}{l}\text { septische Kreislaufsituation; V.a. atypische Pneumonie bds.; } \\
\text { Thrombozytopenie; Refluxösophagitis; erosive Gastritis/ } \\
\text { Bulbitis; chron. Pankreatitis }\end{array}$ \\
\hline 16 & 56 & M & 170 & 90 & $\begin{array}{l}\text { 3-Gefäß-KHK, } \\
\text { Z.n. Reanimation bei Kammerflimmern }\end{array}$ & $\begin{array}{l}\text { art.Hypertonus, IDDM mit Spätsyndrom (Polyneuropathie, } \\
\text { Retinopathie) }\end{array}$ \\
\hline 17 & 51 & M & 175 & 90 & Panzytopenie; septische Kreislauflage & $\begin{array}{l}\text { Z.n. megaloblastärer Anämie mit autoimmunologischer } \\
\text { Komponente }\end{array}$ \\
\hline 18 & 70 & $\mathrm{M}$ & 170 & 85 & $\begin{array}{l}\text { VWI bei 2-Gefäß-KHK, Z.n. Reanimation bei } \\
\text { Kammerflimmern, Lungenödem, rezid. Ventrikuläre } \\
\text { Tachykardie }\end{array}$ & Sepsis bei Aspirationspneumonie, ANV \\
\hline
\end{tabular}

Tabelle 14: Diagnosen und weitere relevante Erkrankungen der Intensivpatienten Teil 1 


\begin{tabular}{|c|c|c|c|c|c|c|}
\hline Pat.Nr. & $\begin{array}{c}\text { Alter } \\
\text { (Jahre) }\end{array}$ & Geschlecht & $\begin{array}{c}\text { Größe } \\
(\mathrm{cm})\end{array}$ & $\begin{array}{c}\text { Gewicht } \\
(\mathrm{kg})\end{array}$ & Diagnose & weitere relevante Erkrankungen \\
\hline 19 & 85 & M & 175 & 80 & $\begin{array}{l}\text { pulmonale Raumforderung (DD: atypische } \\
\text { Pneumonie, M.Wegener, Alveolarzell-CA) }\end{array}$ & $\begin{array}{l}\text { resp. Insuffizienz, ANV, intermitt. Arrhythmia absoluta, } \\
\text { intermitt. AV-Block Grad I-II }\end{array}$ \\
\hline 20 & 33 & M & 182 & 76 & AML-Rezidiv, Blastenschub & $\begin{array}{l}\text { Z.n. Polychemotherapie und KM-Transplantation, } \\
\text { ANV }\end{array}$ \\
\hline 17 & 51 & M & 175 & 90 & Panzytopenie; septische Kreislauflage & Z.n. megaloblastärer Anämie mit autoimmun. Komponente \\
\hline 21 & 72 & $\mathrm{~W}$ & - & - & $\begin{array}{l}\text { LV-Dekompensation bei TAA, ANV, respirst. } \\
\text { Insuff., Lungenödem }\end{array}$ & V.a. COPD bei Nikotinabusus \\
\hline 22 & 53 & M & 172 & 95 & $\begin{array}{l}\text { dilat. Kardiomyopathie, Pneumonie, Sepsis, } \\
\text { resp.Insuffizienz }\end{array}$ & ANV; Z.n.AICD-Implantation; art.Hypertonus \\
\hline 23 & 58 & $\mathrm{~W}$ & 155 & 45 & $\mathrm{CO}_{2}$-Narkose bei infektexazerbierter COPD & $\begin{array}{l}\text { Asthma bronchiale; pulm. Hypertonie; 2-Gefäß KHK; Z.n. } \\
\text { RCA-PTCA; kompensierte Niereninsuffizienz; Sprue }\end{array}$ \\
\hline 24 & 61 & $\mathrm{M}$ & 180 & 80 & $\begin{array}{l}\text { ischämische Kardiomyopathie; } \\
\text { TAA bei VHF u. LSB }\end{array}$ & $\begin{array}{l}\text { kardiog. Schock mit Lungenödem; ANV; Z.n. protrahierter } \\
\text { kardiopulmonaler Reanimation; KHK; V.a. Sepsis; instabile } \\
\text { AP-Symptomatik; Bronchitis }\end{array}$ \\
\hline 25 & 50 & W & 164 & 79 & $\begin{array}{l}\text { metabolisch toxischer Leberparenchymschaden mit } \\
\text { zirrhotischem Umbau und Fettleberhepatitis CHILD- } \\
\mathrm{c} \text { terminales hepatorenales Syndrom }\end{array}$ & $\begin{array}{l}\text { hepatische Enzephalopathie; Ikterus; unklarer Leberrundherd; } \\
\text { Hyperkalzämie; DM; Adipositas; Hypothyreose }\end{array}$ \\
\hline $\begin{array}{l}\text { Median } \\
\text { SD }\end{array}$ & $\begin{array}{c}56 \\
13,5\end{array}$ & $\begin{array}{l}10 \mathrm{M} \\
5 \mathrm{~W}\end{array}$ & $\begin{array}{l}172 \\
7,9\end{array}$ & $\begin{array}{c}80 \\
17,5\end{array}$ & & \\
\hline
\end{tabular}

Tabelle 15: Diagnosen und weitere relevante Erkrankungen der Intensivpatienten Teil 2 


\begin{tabular}{|c|c|c|c|c|c|c|c|c|c|c|c|c|c|c|c|c|c|c|c|c|}
\hline \multirow[t]{2}{*}{ Pat.Nr. } & \multicolumn{3}{|c|}{ Elektrolyte } & \multirow{2}{*}{\begin{tabular}{|c} 
Niere \\
Krea \\
{$[\mathrm{mg} / \mathrm{dl}]$}
\end{tabular}} & \multicolumn{2}{|c|}{ Protein } & \multicolumn{5}{|c|}{ Leberwerte } & \multicolumn{4}{|c|}{ Gerinnung } & \multicolumn{5}{|l|}{ Blutbild } \\
\hline & $\mid \begin{array}{c}N a \\
{[\mathrm{mg} / \mathrm{dl}]}\end{array}$ & $\begin{array}{c}K \\
{[m g / d l]}\end{array}$ & {$\left[\begin{array}{c}\mathrm{Ca} \\
{[\mathrm{mmol} / \mathrm{ll}]}\end{array}\right.$} & & $\begin{array}{l}\text { Prot } \\
{[\mathrm{g} / \mathrm{dl}]}\end{array}$ & $\begin{array}{c}A l b \\
{[g / d l]}\end{array}$ & $\begin{array}{l}A S T \\
{[U / l]}\end{array}$ & $\begin{array}{l}A L T \\
{[U / l]}\end{array}$ & $\begin{array}{l}y-G T \\
{[U / l]}\end{array}$ & $\begin{array}{c}A P \\
{[U / l]}\end{array}$ & {$\left[\begin{array}{c}\text { Bili } \\
{[\mathrm{mg} / \mathrm{dl}]}\end{array}\right.$} & $\begin{array}{c}\text { Quick } \\
{[\%]}\end{array}$ & $\begin{array}{l}\text { PTT } \\
\text { [sek] }\end{array}$ & $\begin{array}{l}\mathrm{Fibr} \\
{[\mathrm{mg} / \mathrm{dl}]}\end{array}$ & $\begin{array}{c}A T \text { III } \\
{[\%]}\end{array}$ & $\begin{array}{l}H b \\
{[\mathrm{~g} / \mathrm{dl}]}\end{array}$ & $\begin{array}{l}H k \\
{[\%]}\end{array}$ & {$\left[\begin{array}{c}\text { Erys } \\
{\left[x 10^{6} / \mu l\right]}\end{array}\right.$} & $\begin{array}{l}\text { Leukos } \\
{\left[x 10^{3} / \mu l\right]}\end{array}$ & $\begin{array}{l}\text { Thrombos } \\
{\left[x 10^{3} / \mu l\right]}\end{array}$ \\
\hline 11 & 150 & 4,1 & 2,4 & 1,3 & 7,3 & 3,3 & 14 & 14 & 30 & 98 & 0,8 & 95 & 28 & 964 & - & 14,4 & 43,5 & 4,35 & 10,8 & 184 \\
\hline 12 & 136 & 4,4 & 2,4 & 1 & 5,4 & 3,2 & 49 & 40 & 11 & 190 & 30,2 & 67 & 49 & 175 & 27 & 9,9 & 28,7 & 2,98 & 10 & 96 \\
\hline 13 & 149 & 4,2 & 1,9 & 0,7 & 5,1 & 2,1 & 18 & 18 & 96 & 278 & 0,4 & 94 & 29 & 428 & 71 & 9,2 & 28,2 & 3,03 & 10,6 & 224 \\
\hline 14 & 137 & 3,7 & 2,3 & 0,8 & 4,2 & 1,8 & 8 & 8 & 9 & 111 & 0,5 & 80 & 73 & 587 & 87 & 11,1 & 33,4 & 3,68 & 9,3 & 15 \\
\hline 15 & 144 & 3,6 & 1,9 & 1,3 & 5,3 & 2 & 64 & 22 & 26 & 143 & 11,1 & 47 & 60 & 483 & 51 & 10 & 29,9 & 3,24 & 39,2 & 111 \\
\hline 16 & - & 4,1 & 2,0 & 1,3 & 6,8 & 2,5 & 312 & 205 & 65 & 123 & 6,9 & 40 & 45 & 939 & 62 & 10,2 & 34,1 & - & 11,2 & 164 \\
\hline 17 & 152 & 5,1 & 1,9 & 1,3 & 5,9 & 2,8 & 31 & 26 & 121 & 681 & 1,9 & 88 & 39 & 502 & 80 & 9,1 & 27,6 & 3,13 & 0,6 & 61 \\
\hline 18 & 149 & 3,6 & 2,1 & 1,5 & 7,1 & 2,8 & 20 & 44 & 31 & 127 & 0,2 & 97 & 51 & 955 & - & 10,4 & 33,2 & 3,42 & 13,5 & 150 \\
\hline 19 & 142 & 4 & 1,7 & 5,6 & 5,8 & 2,7 & 11 & 13 & 67 & 284 & 0,9 & 93 & 32 & 209 & 100 & 11,6 & 33,8 & 3,73 & 6,1 & 17 \\
\hline 20 & 151 & 4,4 & 2,2 & 1,2 & 5,7 & 2,4 & 508 & 443 & 138 & 333 & 2,6 & 64 & 41 & 592 & - & 9,7 & 29,1 & 2,93 & 45,9 & 44 \\
\hline 21 & 138 & 3,5 & 2,3 & 0,6 & 5,1 & 2 & 15 & 9 & 42 & 225 & 2,9 & 93 & 46 & 580 & 51 & 8,7 & 26,1 & 3,05 & 16 & 148 \\
\hline 22 & 140 & 4,4 & 2,0 & 1,4 & 7,2 & 1,6 & 16 & 13 & 285 & 519 & 1,6 & 92 & 43 & 726 & 88 & 10,4 & 32,5 & 3,65 & 14,2 & 188 \\
\hline 23 & 140 & 5,1 & - & 1,6 & 5,1 & 2,2 & 64 & 174 & 348 & 271 & 2,5 & 140 & 26 & 777 & 142 & 9,4 & 27,7 & 2,94 & 24,5 & 59 \\
\hline 24 & 139 & 5,4 & 2,1 & 1,4 & 4,9 & 2 & 680 & 658 & 16 & 88 & 3,1 & 60 & 48 & 677 & 45 & 12 & 35 & 3,42 & 16,5 & 123 \\
\hline 25 & 139 & 3,8 & 2,9 & 3,6 & 6,1 & 2,8 & 16 & 8 & 21 & 221 & 25,1 & 41 & 75 & 171 & 19 & 8,3 & 24 & 2,66 & 21,5 & 39 \\
\hline
\end{tabular}

Tabelle 16: Laborparameter der Intensivpatienten 


\begin{tabular}{|c|c|c|c|c|c|c|c|c|c|c|c|}
\hline Pat.Nr. & $\begin{array}{l}\text { Infekt- } \\
\text { nachweis }\end{array}$ & Fieberursache & Mikrobiologie & $\begin{array}{l}\text { Positive } \\
\text { BK/BAL }\end{array}$ & $\begin{array}{c}\text { Pulmonale-/ } \\
\text { sonstige } \\
\text { Infektion } \\
\end{array}$ & $\begin{array}{l}\text { Leukos } \\
{[\mathrm{Tsd} / \mu \mathrm{l}]}\end{array}$ & $\begin{array}{l}\mathrm{CRP} \\
{[\mathrm{mg} / \mathrm{l}]}\end{array}$ & $\begin{array}{l}\text { Temp } \\
{\left[{ }^{\circ} \mathrm{C}\right]}\end{array}$ & $\begin{array}{c}\mathrm{HF} \\
{\left[\mathrm{min}^{-1}\right]}\end{array}$ & $\begin{array}{c}\mathrm{AF} \\
{\left[\mathrm{min}^{-1}\right]}\end{array}$ & $\begin{array}{l}\text { RRsyst } \\
{[\mathrm{mmHg}]}\end{array}$ \\
\hline 11 & CMDI & $\begin{array}{l}\text { Stauungspneumonie } \\
\text { mit Sepsis }\end{array}$ & Bronchialsekret: Staph.aureus & - & $\mathrm{Ja}$ & 10,8 & 230,2 & 39,0 & 90 & BIPAP & 110 \\
\hline 12 & $\mathrm{CDI}$ & $\begin{array}{l}\text { V.a.Cholangitis mit system. } \\
\text { Reaktion }\end{array}$ & & - & - & 10,0 & 31,8 & 38,7 & 95 & BIPAP & - \\
\hline 13 & CMDI & $\begin{array}{l}\text { Infizierte Laparotomie- } \\
\text { wunde, HWI }\end{array}$ & $\begin{array}{l}\text { Urin-Kultur: Enterokokkus Gr.D; } \\
\text { Wundabstrich: Enterobacter spec., } \\
\text { E.faecalis }\end{array}$ & - & - & 15,7 & 111,0 & 38,6 & 100 & - & 150 \\
\hline 14 & CMDI & $\begin{array}{l}\text { Toxic shock bei } \\
\text { nekrotisierender Fasciitis }\end{array}$ & $\begin{array}{l}\text { Biopsat re Mamma: Streptokokkus } \\
\text { pyogenes Gr. A }\end{array}$ & - & $\mathrm{Ja}$ & 9,3 & 218,1 & 37,0 & 80 & 16 & 120 \\
\hline 15 & CMDI & Sepsis bei Pneumonie & Bronchialsekret: Enterococcus spec. & $\mathrm{Ja}$ & $\mathrm{Ja}$ & 39,2 & 253,8 & 38,7 & 103 & BIPAP & 100 \\
\hline 16 & CMDI & Sepsis & Blutkultur: gram.pos.Kokken & $\mathrm{Ja}$ & $\mathrm{Ja}$ & 11,2 & 254,0 & 39,7 & 79 & BIPAP & 127 \\
\hline 17 & CDI & $\begin{array}{l}\text { Sepsis unklarer Genese; } \\
\text { Panzytopenie }\end{array}$ & & - & $\mathrm{Ja}$ & 0,9 & 126,6 & 39,2 & 90 & BIPAP & 90 \\
\hline 18 & CMDI & $\begin{array}{l}\text { Sepsis bei Aspirations- } \\
\text { pneumonie }\end{array}$ & $\begin{array}{l}\text { ZVK-Spitze: P.aeruginosa } \\
\text { Bronchialsekret: P.aeruginosa }\end{array}$ & $\mathrm{Ja}$ & - & 16,9 & 99,4 & 38,8 & 100 & BIPAP & 110 \\
\hline
\end{tabular}

Tabelle 17: Infektionsparameter der Intensivpatienten Teil 1 


\begin{tabular}{|c|c|c|c|c|c|c|c|c|c|c|c|}
\hline Pat.Nr. & $\begin{array}{l}\text { Infekt- } \\
\text { nachweis }\end{array}$ & Fieberursache & Mikrobiologie & $\begin{array}{l}\text { Positive } \\
\text { BK/BAL }\end{array}$ & $\begin{array}{l}\text { Pulmonale- } \\
\text { / sonstige } \\
\text { Infektion }\end{array}$ & $\begin{array}{l}\text { Leukos } \\
{[\mathrm{Tsd} / \mu \mathrm{l}]}\end{array}$ & $\begin{array}{c}\mathrm{CRP} \\
{[\mathrm{mg} / \mathrm{l}]}\end{array}$ & $\begin{array}{l}\text { Temp } \\
{\left[{ }^{\circ} \mathrm{C}\right]}\end{array}$ & $\begin{array}{c}\mathrm{HF} \\
{\left[\mathrm{min}^{-1}\right]}\end{array}$ & $\mathrm{AF}$ & $\begin{array}{l}\text { RRsyst } \\
{[\mathrm{mmHg}]}\end{array}$ \\
\hline 18 & CMDI & $\begin{array}{l}\text { Sepsis bei Aspirations- } \\
\text { pneumonie }\end{array}$ & $\begin{array}{l}\text { ZVK-Spitze: P.aeruginosa } \\
\text { Bronchialsekret: P.aeruginosa }\end{array}$ & $\mathrm{Ja}$ & - & 16,9 & 99,4 & 38,8 & 100 & BIPAP & 110 \\
\hline 19 & CMDI & Stauungspneumonie & $\begin{array}{l}\text { Bronchialsekret: Cand.albicans, } \\
\text { E.faecalis; BAL: Cand.albicans, } \\
\text { Staph.epidermidis }\end{array}$ & $\mathrm{Ja}$ & $\mathrm{Ja}$ & 6,0 & 23,4 & 38,8 & 100 & BIPAP & 110 \\
\hline 20 & CDI & $\begin{array}{l}\text { Sepsis bei Blastenschub einer } \\
\text { AML }\end{array}$ & & - & - & 45,9 & 463,5 & 39,8 & 100 & 20 & 90 \\
\hline 21 & CMDI & Sepsis & Blutkultur: Staph.epid. & $\mathrm{Ja}$ & $\mathrm{Ja}$ & 16,0 & 216,0 & 39,2 & 90 & BIPAP & 120 \\
\hline 22 & CMDI & Sepsis bei Pneumonie & $\begin{array}{l}\text { Katheterspitze: koag.neg.Staph, } \\
\text { Bronchiallavage: Hefen, Enterococcus, } \\
\text { Staph aureus, Blutkultur:koag.neg. } \\
\text { Staph., Sheldonspitze: koag.neg.Staph }\end{array}$ & $\mathrm{Ja}$ & $\mathrm{Ja}$ & 14,2 & 186,4 & 39,1 & 130 & BIPAP & 100 \\
\hline 23 & CMDI & Sepsis bei Pneumonie & $\begin{array}{l}\text { Bronchialsekret: Hefen, E.faecalis, } \\
\text { Sheldonspitze: E. faecalis }\end{array}$ & $\mathrm{Ja}$ & $\mathrm{Ja}$ & 24,5 & 70,0 & 37,9 & 100 & 15 & 160 \\
\hline 24 & $\mathrm{CDI}$ & V.a. Sepsis & & - & $\mathrm{Ja}$ & 16,5 & 288,1 & 38,4 & 90 & BIPAP & 100 \\
\hline 25 & CMDI & Sepsis bei Wundinfektion & $\begin{array}{l}\text { Sheldon-Spitze: E.faecalis; Abstrich- } \\
\text { Decubitus: Staph.epidermidids, Hefen, } \\
\text { E.faecalis }\end{array}$ & $\mathrm{Ja}$ & - & 21,5 & 55,9 & 35,2 & 100 & BIPAP & 100 \\
\hline
\end{tabular}

Tabelle 18: Infektionsparameter der Intensivpatienten Teil 2 


\begin{tabular}{|c|c|c|c|c|c|c|c|c|}
\hline Pat.Nr. & SIRS & $\begin{array}{l}\text { Sepsis- } \\
\text { kriterien }\end{array}$ & Organdysfunktion & Hypoperfusion & Hypotension & & & $\begin{array}{l}\text { Schweregrad der } \\
\text { Infektion }\end{array}$ \\
\hline & & & $\begin{array}{l}\text { Schocklunge, ANV, } \\
\text { Multiorganversagen }\end{array}$ & $\begin{array}{l}\text { Lactatacidose, } \\
\text { oder Abfall des } \\
\text { Status }\end{array}$ & $\begin{array}{l}\text { RR-Abfall }<90 \mathrm{mmHg} \\
\text { oder um mehr als } \\
40 \mathrm{mmHg}\end{array}$ & $\begin{array}{l}\text { Notwendigkeit positiv } \\
\text { inotroper Substanzen }\end{array}$ & $\begin{array}{l}\text { Notwendigkeit } \\
\text { forcierter } \\
\text { Flüssigkeitszufuhr }\end{array}$ & \\
\hline 11 & 3 & $\mathrm{Ja}$ & nein & Nein & nein & ja (bei akuter HI) & Nein & Sepsis \\
\hline 12 & 4 & Nein & nein & Nein & nein & Nein & Nein & V.a. Sepsis \\
\hline 13 & 4 & $\mathrm{Ja}$ & nein & Nein & nein & nein & Nein & Sepsis \\
\hline 14 & 0 & Nein & nein & Nein & nein & nein & Nein & keine Sepsis \\
\hline 15 & 3 & $\mathrm{Ja}$ & $\mathrm{Ja}$ & Ja (Lactatacidose) & ja & ja & $\mathrm{Ja}$ & septischer Schock \\
\hline 16 & 2 & $\mathrm{Ja}$ & $\mathrm{Ja}$ & $\mathrm{Ja}$ (Lactatacidose) & nein & ja & $\mathrm{Ja}$ & septischer Schock \\
\hline 17 & 2 & $\mathrm{Ja}$ & $\mathrm{Ja}$ & $\mathrm{Ja}$ (Oligurie) & ja & ja & $\mathrm{Ja}$ & septischer Schock \\
\hline 18 & 4 & $\mathrm{Ja}$ & $\mathrm{Ja}$ & Ja (Oligurie) & nein & nein & Nein & Sepsis \\
\hline 19 & 2 & $\mathrm{Ja}$ & $\mathrm{Ja}(\mathrm{ANV})$ & Ja (Oligurie) & nein & ja & $\mathrm{Ja}$ & septischer Schock \\
\hline 20 & 4 & $\mathrm{Ja}$ & $\mathrm{Ja}(\mathrm{ANV})$ & Ja (Lactatacidose) & ja & nein & $\mathrm{Ja}$ & septischer Schock \\
\hline 21 & 3 & $\mathrm{Ja}$ & $\mathrm{Ja}(\mathrm{ANV})$ & Ja (Z.n.ANV) & nein & ja & $\mathrm{Ja}$ & septischer Schock \\
\hline 22 & 2 & $\mathrm{Ja}$ & $\mathrm{Ja}(\mathrm{ANV})$ & Ja (Oligurie) & ja & ja (bei akuter HI) & Nein & septischer Schock \\
\hline 23 & 3 & $\mathrm{Ja}$ & $\mathrm{Ja}(\mathrm{ANV})$ & Ja (Oligurie) & nein & nein & Nein & Sepsis \\
\hline 24 & 2 & $\mathrm{Ja}$ & $\mathrm{Ja}(\mathrm{ANV})$ & Ja (Oligurie) & nein & ja (bei akuter HI) & Nein & Sepsis \\
\hline 25 & 3 & $\mathrm{Ja}$ & $\mathrm{Ja}(\mathrm{ANV})$ & $\mathrm{Ja}$ (Lactatacidose) & nein & ja & Nein & septischer Schock \\
\hline
\end{tabular}

Tabelle 19: Schweregrad der Infektion der Intensivpatienten 


\begin{tabular}{|c|c|c|c|c|c|c|c|c|c|c|c|c|c|}
\hline Pat.Nr. & Geschlecht & $\begin{array}{c}\text { Gewicht } \\
(\mathrm{kg})\end{array}$ & $\begin{array}{c}\text { Größe } \\
(\mathrm{cm})\end{array}$ & $\begin{array}{c}\text { Alter } \\
\text { (Jahre) }\end{array}$ & $\begin{array}{c}\text { Krea } \\
(\mathrm{mg} / \mathrm{dl})\end{array}$ & $\begin{array}{l}\text { IBW } \\
(\mathrm{kg})\end{array}$ & $\begin{array}{c}\mathrm{Cl}_{\text {krea }} \\
(\mathrm{ml} / \mathrm{min})\end{array}$ & $\begin{array}{r}\text { Dosis } \\
(\mathrm{mg})\end{array}$ & $\begin{array}{l}\text { Intervall- } \\
\text { dauer (h) }\end{array}$ & $\begin{array}{l}\text { Infusions- } \\
\text { intervall (n) }\end{array}$ & $\begin{array}{c}\text { Infusions- } \\
\text { dauer (h) }\end{array}$ & $\begin{array}{c}\mathrm{C}_{\max } \\
(\mathrm{mg} / \mathrm{l})\end{array}$ & $\begin{array}{c}\mathrm{C}_{\min } \\
(\mathrm{mg} / \mathrm{l})\end{array}$ \\
\hline 11 & $\mathrm{M}$ & 128,5 & 182 & 51 & 1,3 & 82,86 & 78,79 & 500 & 8 & 4 & 0,22 & 24,14 & 0,02 \\
\hline 12 & M & 91 & 165 & 74 & 1,0 & 66,90 & 43,8 & 500 & 8 & 2 & 0,55 & 9,60 & 2,40 \\
\hline 13 & $\mathrm{~W}$ & 72 & 160 & 59 & 0,7 & 57,70 & 78,83 & 1000 & 8 & 4 & 0,33 & 44,69 & 0,62 \\
\hline 14 & $\mathrm{~W}$ & 73 & 172 & 42 & 0,8 & 68,97 & 113,99 & 1000 & 8 & 8 & 0,25 & 39,49 & 2,85 \\
\hline 15 & M & 80 & 175 & 45 & 1,3 & 76,29 & 77,43 & 1000 & 8 & 2 & 0,50 & 45,28 & 5,78 \\
\hline 16 & M & 90 & 170 & 56 & 1,3 & 71,59 & 55,68 & 500 & 8 & 1 & 0,17 & 19,82 & 2,47 \\
\hline 17 & M & 90 & 175 & 51 & 1,3 & 76,29 & 72,54 & 500 & 12 & 23 & 0,33 & 13,46 & $0,70 / 0,42$ \\
\hline 18 & M & 85 & 170 & 70 & 1,5 & 71,59 & 63,28 & 500 & 8 & 12 & 0,42 & 27,24 & 0,95 \\
\hline 19 & $\mathrm{M}$ & 80 & 175 & 85 & 5,6 & 76,29 & 10,41 & 500 & 8 & 2 & 0,70 & 41,33 & 11,20 \\
\hline 20 & M & 76 & 182 & 33 & 1,2 & 82,86 & Z.n.CVVHF & 500 & 8 & 10 & 1,32 & 10,75 & 2,30 \\
\hline 21 & $\mathrm{~W}$ & 70 & 165 & 72 & 0,6 & 62,40 & Z.n.CVVHF & 1000 & 8 & 12 & 0,17 & 50,75 & 13,70 \\
\hline 22 & M & 95 & 172 & 53 & 1,4 & 73,47 & CVVHF & 1000 & 8 & 4 & 0,217 & 47,56 & 7,03 \\
\hline 23 & W & 45 & 155 & 58 & 1,6 & 53,01 & CVVHF & 1000 & 12 & 1 & 0,167 & 70,54 & 14,20 \\
\hline 24 & M & 80 & 180 & 61 & 1,4 & 80,98 & CVVHF & 1000 & 12 & 1 & 0,167 & 66,84 & 5,04 \\
\hline 25 & $\mathrm{~W}$ & 79 & 164 & 50 & 3,6 & 61,46 & CVVHF & 500 & 8 & 5 & 0,533 & 15,80 & 3,68 \\
\hline Mittelwert & & 82,3 & 170,8 & 57,3 & 1,6 & 70,84 & 66,08 & 733,3 & 8,8 & 6,1 & 0,40 & 35,15 & 5,16 \\
\hline Median & & 80,0 & 172,0 & 56,0 & 1,3 & 71,59 & 72,54 & 500,0 & 8,0 & 4,0 & 0,33 & 39,49 & 3,27 \\
\hline SD & & 17,5 & 7,9 & 13,5 & 1,3 & 9,09 & 28,51 & 258,2 & 1,7 & 6,1 & 0,30 & 19,67 & 4,73 \\
\hline Min & & 45,0 & 155,0 & 33,0 & 0,6 & 53,01 & 10,41 & 500,0 & 8,0 & 1,0 & 0,17 & 9,60 & 0,02 \\
\hline Max & & 128,5 & 182,0 & 85,0 & 5,6 & 82,86 & 113,99 & 1000,0 & 12,0 & 23,0 & 1,32 & 70,54 & 14,20 \\
\hline
\end{tabular}

Tabelle 20: Pharmakokinetische Parameter der Intensivpatienten Teil 1 


\begin{tabular}{|c|c|c|c|c|c|c|c|c|c|c|c|c|}
\hline Pat.Nr. & $\begin{array}{c}\mathrm{Cl}_{\text {mer }} \\
(\mathrm{ml} / \mathrm{min})\end{array}$ & $\begin{array}{l}\mathrm{V}_{\mathrm{ss}} \\
\text { (1) }\end{array}$ & $\begin{array}{c}\mathrm{V}_{\mathrm{ss}} \\
(1 / \mathrm{kg})\end{array}$ & $\begin{array}{l}\text { MRT } \\
(\min )\end{array}$ & $\begin{array}{c}\mathrm{k}_{\mathrm{el}} \\
(1 / \mathrm{h})\end{array}$ & $\begin{array}{c}\mathrm{T}_{1 / 2 \alpha} \\
\text { (h) }\end{array}$ & $\begin{array}{c}\mathrm{T}_{1 / 2 \beta} \\
(\mathrm{h})\end{array}$ & $\begin{array}{l}\mathrm{AUC}_{0-\infty} \\
\left(\mathrm{mg}^{*} \mathrm{~h} / \mathrm{l}\right)\end{array}$ & $\begin{array}{c}\mathrm{T}>2 \mathrm{mg} / \mathrm{l} \\
(\mathrm{h})\end{array}$ & $\begin{array}{c}\mathrm{T}>4 \mathrm{mg} / 1 \\
\text { (h) }\end{array}$ & $\begin{array}{c}\mathrm{T}>2 \mathrm{mg} / \mathrm{l} \\
(\%)\end{array}$ & $\begin{array}{c}\mathrm{T}>4 \mathrm{mg} / 1 \\
(\%)\end{array}$ \\
\hline 11 & 311,3 & 19,51 & 0,15 & 62,7 & 0,96 & 0,45 & 0,80 & 24,010 & 3,37 & 2,40 & 42,07 & 30,01 \\
\hline 12 & 157,3 & 52,21 & 0,57 & 331,9 & 0,18 & 0,38 & 3,95 & 49,97 & 9,53 & 5,45 & 100,00 & 68,08 \\
\hline 13 & 245,6 & 28,61 & 0,40 & 116,5 & 0,52 & 0,31 & 1,65 & 59,928 & 6,63 & 3,92 & 82,87 & 49,02 \\
\hline 14 & 162,2 & 36,11 & 0,49 & 222,6 & 0,27 & 0,43 & 2,93 & 97,623 & 9,72 & 7,17 & 100,00 & 89,59 \\
\hline 15 & 99,1 & 25,45 & 0,32 & 256,9 & 0,23 & 0,52 & 3,19 & 156,376 & 18,91 & 13,35 & 100,00 & 100,00 \\
\hline 16 & 124,0 & 48,09 & 0,53 & 388,0 & 0,15 & 0,46 & 5,68 & 67,23 & 8,29 & 4,8 & 100 & 60,04 \\
\hline 17 & 398,6 & 69,42 & 0,77 & 174,2 & 0,34 & 0,12 & 2,37 & 18,241 & 3,73 & 1,41 & 31,09 & 11,75 \\
\hline 18 & 137,0 & 22,57 & 0,27 & 164,8 & 0,36 & 0,69 & 2,51 & 54,833 & 6,77 & 4,67 & 84,66 & 58,38 \\
\hline 19 & 30,4 & 17,50 & 0,22 & 575,8 & 0,10 & 0,54 & 7,21 & 258,24 & 23,06 & 17,26 & 100,00 & 100,00 \\
\hline 20 & 147,6 & 44,32 & 0,58 & 300,4 & 0,20 & 0,96 & 3,69 & 48,92 & 8,62 & 6,32 & 100,00 & 78,96 \\
\hline 21 & 56,6 & 25,19 & 0,36 & 445,0 & 0,13 & 0,62 & 5,38 & 290,04 & 20,23 & 15,56 & 100,00 & 100,00 \\
\hline 22 & 98,4 & 32,022 & 0,34 & 325,55 & 0,18 & 0,344 & 4,05 & 169,44 & 14,09 & 10,54 & 100 & 100 \\
\hline 23 & 50,9 & 21,773 & 0,48 & 428,18 & 0,14 & 0,184 & 5,27 & 327,76 & 25,9 & 19,72 & 100 & 100 \\
\hline 24 & 121,1 & 30,92 & 0,39 & 255,28 & 0,24 & 0,172 & 3,28 & 137,60 & 11,39 & 8,42 & 94,93 & 70,15 \\
\hline 25 & 87,1 & 44,111 & 0,56 & 506,20 & 0,12 & 0,723 & 6,37 & 95,63 & 16,17 & 8,86 & 100 & 100 \\
\hline Mittelwert & 148,48 & 34,52 & 0,43 & 303,6 & 0,27 & 0,46 & 3,89 & 123,72 & 12,43 & 8,66 & 89,04 & 74,40 \\
\hline Median & 124,00 & 30,92 & 0,40 & 300,4 & 0,20 & 0,45 & 3,69 & 95,63 & 9,72 & 7,17 & 100,00 & 78,96 \\
\hline SD & 100,31 & 14,51 & 0,16 & 146,2 & 0,22 & 0,23 & 1,80 & 98,68 & 7,00 & 5,56 & 22,13 & 28,34 \\
\hline Min & 30,40 & 17,50 & 0,15 & 62,7 & 0,10 & 0,12 & 0,80 & 18,24 & 3,37 & 1,41 & 31,09 & 11,75 \\
\hline Max & 398,60 & 69,42 & 0,77 & 575,8 & 0,96 & 0,96 & 7,21 & 327,76 & 25,90 & 19,72 & 100,00 & 100,00 \\
\hline
\end{tabular}

Tabelle 21: Pharmakokinetische Parameter der Intensivpatienten Teil 2 


\begin{tabular}{|c|c|c|c|c|c|c|c|c|c|c|c|c|c|}
\hline Pat.Nr. & Geschlecht & $\begin{array}{l}\text { Gewicht } \\
(\mathrm{kg})\end{array}$ & $\begin{array}{l}\text { Größe } \\
(\mathrm{cm})\end{array}$ & $\begin{array}{c}\text { Alter } \\
\text { (Jahre) }\end{array}$ & $\begin{array}{c}\text { Krea } \\
(\mathrm{mg} / \mathrm{dl})\end{array}$ & $\begin{array}{l}\text { IBW } \\
(\mathrm{kg})\end{array}$ & $\begin{array}{c}\mathrm{Cl}_{\text {krea }} \\
(\mathrm{ml} / \mathrm{min})\end{array}$ & $\begin{array}{r}\text { Dosis } \\
(\mathrm{mg})\end{array}$ & $\begin{array}{l}\text { Intervall- } \\
\text { dauer (h) }\end{array}$ & $\begin{array}{l}\text { Infusions- } \\
\text { intervall (n) }\end{array}$ & $\begin{array}{l}\text { Infusions- } \\
\text { dauer (h) }\end{array}$ & $\begin{array}{c}\mathrm{C}_{\max } \\
(\mathrm{mg} / \mathrm{l})\end{array}$ & $\begin{array}{l}\mathrm{C}_{\min } \\
(\mathrm{mg} / \mathrm{l})\end{array}$ \\
\hline 11 & M & 128,5 & 182 & 51 & 1,3 & 82,86 & 78,79 & 500 & 8 & 4 & 0,22 & 24,14 & 0,02 \\
\hline 13 & W & 72 & 160 & 59 & 0,7 & 57,70 & 78,83 & 1000 & 8 & 4 & 0,33 & 44,69 & 0,62 \\
\hline 14 & W & 73 & 172 & 42 & 0,8 & 68,97 & 113,99 & 1000 & 8 & 8 & 0,25 & 39,49 & 2,85 \\
\hline 15 & M & 80 & 175 & 45 & 1,3 & 76,29 & 77,43 & 1000 & 8 & 2 & 0,50 & 45,28 & 5,78 \\
\hline 17 & M & 90 & 175 & 51 & 1,3 & 76,29 & 72,54 & 500 & 12 & 23 & 0,33 & 13,46 & $0,70 / 0,42$ \\
\hline 18 & M & 85 & 170 & 70 & 1,5 & 71,59 & 63,28 & 500 & 8 & 12 & 0,42 & 27,24 & 0,95 \\
\hline Mittelwert & & 88,1 & 172,3 & 53,0 & 1,2 & 72,28 & 80,81 & 750 & 8,7 & 8,8 & 0,34 & 32,38 & 2,04 \\
\hline Median & & 82,5 & 173,5 & 51,0 & 1,3 & 73,94 & 78,11 & 750 & 8,0 & 6,0 & 0,33 & 33,37 & 0,95 \\
\hline SD & & 21,0 & 7,3 & 10,2 & 0,3 & 8,58 & 17,30 & 274 & 1,6 & 7,8 & 0,10 & 12,81 & 2,34 \\
\hline Min & & 72,0 & 160,0 & 42,0 & 0,7 & 57,70 & 63,28 & 500 & 8,0 & 2,0 & 0,22 & 13,46 & 0,02 \\
\hline Max & & 128,5 & 182,0 & 70,0 & 1,5 & 82,86 & 113,99 & 1000 & 12,0 & 23,0 & 0,50 & 45,28 & 5,78 \\
\hline
\end{tabular}

Tabelle 22: Pharmakokinetische Parameter der Intensivpatienten ohne Nierenfunktionseinschränkungen Teil 1 


\begin{tabular}{|c|c|c|c|c|c|c|c|c|c|c|c|c|}
\hline Pat.Nr. & $\begin{array}{c}\mathrm{Cl}_{\text {mer }} \\
(\mathrm{ml} / \mathrm{min})\end{array}$ & $\begin{array}{l}\mathrm{V}_{\mathrm{ss}} \\
\text { (l) }\end{array}$ & $\begin{array}{c}\mathrm{V}_{\mathrm{ss}} \\
(1 / \mathrm{kg})\end{array}$ & $\begin{array}{l}\text { MRT } \\
(\min )\end{array}$ & $\begin{array}{c}\mathrm{k}_{\mathrm{el}} \\
(1 / \mathrm{h})\end{array}$ & $\begin{array}{c}\mathrm{T}_{1 / 2 \alpha} \\
(\mathrm{h})\end{array}$ & $\begin{array}{c}\mathrm{T}_{1 / 2 \beta} \\
\text { (h) }\end{array}$ & $\begin{array}{c}\mathrm{AUC}(0- \\
\infty) \\
\left(\mathrm{mg}^{*} \mathrm{~h} / 1\right)\end{array}$ & $\begin{array}{c}\mathrm{T}>2 \mathrm{mg} / 1 \\
(\mathrm{~h})\end{array}$ & $\begin{array}{c}\mathrm{T}>4 \mathrm{mg} / 1 \\
(\mathrm{~h})\end{array}$ & $\begin{array}{c}\mathrm{T}>2 \mathrm{mg} / 1 \\
(\%)\end{array}$ & $\begin{array}{c}\mathrm{T}>4 \mathrm{mg} / 1 \\
(\%)\end{array}$ \\
\hline 11 & 311,3 & 19,51 & 0,15 & 62,7 & 0,96 & 0,45 & 0,80 & 24,010 & 3,37 & 2,40 & 42,07 & 30,01 \\
\hline 13 & 245,6 & 28,61 & 0,40 & 116,5 & 0,52 & 0,31 & 1,65 & 59,928 & 6,63 & 3,92 & 82,87 & 49,02 \\
\hline 14 & 162,2 & 36,11 & 0,49 & 222,6 & 0,27 & 0,43 & 2,93 & 97,623 & 9,72 & 7,17 & 100,00 & 89,59 \\
\hline 15 & 99,1 & 25,45 & 0,32 & 256,9 & 0,23 & 0,52 & 3,19 & 156,376 & 18,91 & 13,35 & 100,00 & 100,00 \\
\hline 17 & 398,6 & 69,42 & 0,77 & 174,2 & 0,34 & 0,12 & 2,37 & 18,241 & 3,73 & 1,41 & 31,09 & 11,75 \\
\hline 18 & 137,0 & 22,57 & 0,27 & 164,8 & 0,36 & 0,69 & 2,51 & 54,833 & 6,77 & 4,67 & 84,66 & 58,38 \\
\hline Mittelwert & 225,6 & 33,61 & 0,40 & 166,3 & 0,45 & 0,42 & 2,24 & 68,50 & 8,19 & 5,49 & 73,45 & 56,46 \\
\hline Median & 203,9 & 27,03 & 0,36 & 169,5 & 0,35 & 0,44 & 2,44 & 57,38 & 6,70 & 4,30 & 83,77 & 53,70 \\
\hline SD & 114,5 & 18,44 & 0,21 & 70,2 & 0,27 & 0,19 & 0,88 & 51,64 & 5,74 & 4,33 & 29,68 & 33,91 \\
\hline Min & 99,1 & 19,51 & 0,15 & 62,7 & 0,23 & 0,12 & 0,80 & 18,24 & 3,37 & 1,41 & 31,09 & 11,75 \\
\hline Max & 398,6 & 69,42 & 0,77 & 256,9 & 0,96 & 0,69 & 3,19 & 156,38 & 18,91 & 13,35 & 100,00 & 100,00 \\
\hline
\end{tabular}

Tabelle 23: Pharmakokinetische Parameter der Intensivpatienten ohne Nierenfunktionseinschränkungen Teil 2 


\begin{tabular}{|c|c|c|c|c|c|c|c|c|c|c|c|c|c|}
\hline Pat.Nr. & Geschlecht & $\begin{array}{c}\text { Gewicht } \\
(\mathrm{kg})\end{array}$ & $\begin{array}{c}\text { Größe } \\
(\mathrm{cm})\end{array}$ & $\begin{array}{c}\text { Alter } \\
\text { (Jahre) } \\
\end{array}$ & $\begin{array}{c}\text { Krea } \\
(\mathrm{mg} / \mathrm{dl}) \\
\end{array}$ & $\begin{array}{l}\text { IBW } \\
(\mathrm{kg}) \\
\end{array}$ & $\begin{array}{c}\mathrm{Cl}_{\text {krea }} \\
(\mathrm{ml} / \mathrm{min}) \\
\end{array}$ & $\begin{array}{r}\text { Dosis } \\
(\mathrm{mg}) \\
\end{array}$ & $\begin{array}{l}\text { Intervall- } \\
\text { dauer (h) }\end{array}$ & $\begin{array}{c}\text { Infusions- } \\
\text { intervall (n) }\end{array}$ & \begin{tabular}{|c|} 
Infusions- \\
dauer (h)
\end{tabular} & $\begin{array}{c}\mathrm{C}_{\max } \\
(\mathrm{mg} / \mathrm{l}) \\
\end{array}$ & $\begin{array}{c}\mathrm{C}_{\min } \\
(\mathrm{mg} / \mathrm{l}) \\
\end{array}$ \\
\hline 12 & M & 91 & 165 & 74 & 1,0 & 66,90 & 43,8 & 500 & 8 & 2 & 0,55 & 9,60 & 2,40 \\
\hline 16 & M & 90 & 170 & 56 & 1,3 & 71,59 & 55,68 & 500 & 8 & 1 & 0,17 & 19,82 & 2,47 \\
\hline 19 & M & 80 & 175 & 85 & 5,6 & 76,29 & 10,41 & 500 & 8 & 2 & 0,70 & 41,33 & 11,20 \\
\hline 20 & M & 76 & 182 & 33 & 1,2 & 82,86 & Z.n.CVVHF & 500 & 8 & 10 & 1,32 & 10,75 & 2,30 \\
\hline 21 & $\mathrm{~W}$ & 70 & 165 & 72 & 0,6 & 62,40 & Z.n.CVVHF & 1000 & 8 & 12 & 0,17 & 50,75 & 13,70 \\
\hline 22 & $\mathrm{M}$ & 95 & 172 & 53 & 1,4 & 73,47 & CVVHF & 1000 & 8 & 4 & 0,217 & 47,56 & 7,03 \\
\hline 23 & $\mathrm{~W}$ & 45 & 155 & 58 & 1,6 & 53,01 & CVVHF & 1000 & 12 & 1 & 0,167 & 70,54 & 14,20 \\
\hline 24 & M & 80 & 180 & 61 & 1,4 & 80,98 & CVVHF & 1000 & 12 & 1 & 0,167 & 66,84 & 5,04 \\
\hline 25 & W & 79 & 164 & 50 & 3,6 & 61,46 & CVVHF & 500 & 8 & 5 & 0,533 & 15,80 & 3,68 \\
\hline \begin{tabular}{|c|} 
Mittelwer \\
$\mathrm{t}$
\end{tabular} & & 78,4 & 169,8 & 60,2 & 2,0 & 69,88 & 36,63 & 722,2 & 8,9 & 4,2 & 0,44 & 37,00 & 6,89 \\
\hline Median & & 80,0 & 170,0 & 58,0 & 1,4 & 71,59 & 43,80 & 500,0 & 8,0 & 2,0 & 0,22 & 41,33 & 5,04 \\
\hline SD & & 14,9 & 8,5 & 15,3 & 1,6 & 9,80 & 23,47 & 263,5 & 1,8 & 4,1 & 0,39 & 23,77 & 4,91 \\
\hline Min & & 45,0 & 155,0 & 33,0 & 0,6 & 53,01 & 10,41 & 500,0 & 8,0 & 1,0 & 0,17 & 9,60 & 2,30 \\
\hline Max & & 95,0 & 182,0 & 85,0 & 5,6 & 82,86 & 55,68 & 1000,0 & 12,0 & 12,0 & 1,32 & 70,54 & 14,20 \\
\hline
\end{tabular}

Tabelle 24: Pharmakokinetische Parameter der Intensivpatienten mit Nierenfunktionseinschränkung Teil 1 


\begin{tabular}{|c|c|c|c|c|c|c|c|c|c|c|c|c|}
\hline Pat.Nr. & $\underset{(\mathrm{ml} / \mathrm{min})}{\mathrm{Cl}_{\text {mer }}}$ & $\begin{array}{l}\mathrm{V}_{\mathrm{ss}} \\
\text { (l) }\end{array}$ & $\begin{array}{c}\mathrm{V}_{\mathrm{ss}} \\
(1 / \mathrm{kg})\end{array}$ & $\begin{array}{l}\text { MRT } \\
(\min )\end{array}$ & $\begin{array}{l}\mathrm{k}_{\mathrm{el}} \\
(1 / \mathrm{h})\end{array}$ & $\begin{array}{l}\mathrm{T}_{1 / 2 \alpha} \\
(\mathrm{h})\end{array}$ & $\begin{array}{c}\mathrm{T}_{1 / 2 \beta} \\
\text { (h) }\end{array}$ & $\begin{array}{c}\operatorname{AUC}(0-\infty) \\
(\mathrm{mg} \cdot \mathrm{h} / \mathrm{l})\end{array}$ & $\begin{array}{c}\mathrm{T}>2 \mathrm{mg} / \mathrm{l} \\
\text { (h) }\end{array}$ & $\begin{array}{c}\mathrm{T}>4 \mathrm{mg} / \mathrm{l} \\
\text { (h) }\end{array}$ & $\begin{array}{c}\mathrm{T}>2 \mathrm{mg} / \mathrm{l} \\
(\%)\end{array}$ & $\begin{array}{c}\mathrm{T}>4 \mathrm{mg} / \mathrm{l} \\
(\%)\end{array}$ \\
\hline 12 & 157,3 & 52,21 & 0,57 & 331,9 & 0,18 & 0,38 & 3,95 & 49,97 & 9,53 & 5,45 & 100,00 & 68,08 \\
\hline 16 & 124,0 & 48,09 & 0,53 & 388,0 & 0,15 & 0,46 & 5,68 & 67,23 & 8,29 & 4,8 & 100 & 60,04 \\
\hline 19 & 30,4 & 17,50 & 0,22 & 575,8 & 0,10 & 0,54 & 7,21 & 258,24 & 23,06 & 17,26 & 100,00 & 100,00 \\
\hline 20 & 147,6 & 44,32 & 0,58 & 300,4 & 0,20 & 0,96 & 3,69 & 48,92 & 8,62 & 6,32 & 100,00 & 78,96 \\
\hline 21 & 56,6 & 25,19 & 0,36 & 445,0 & 0,13 & 0,62 & 5,38 & 290,04 & 20,23 & 15,56 & 100,00 & 100,00 \\
\hline 22 & 98,4 & 32,022 & 0,34 & 325,55 & 0,18 & 0,344 & 4,05 & 169,44 & 14,09 & 10,54 & 100 & 100 \\
\hline 23 & 50,9 & 21,773 & 0,48 & 428,18 & 0,14 & 0,184 & 5,27 & 327,76 & 25,9 & 19,72 & 100 & 100 \\
\hline 24 & 121,1 & 30,92 & 0,39 & 255,28 & 0,24 & 0,172 & 3,28 & 137,60 & 11,39 & 8,42 & 94,93 & 70,15 \\
\hline 25 & 87,1 & 44,111 & 0,56 & 506,20 & 0,12 & 0,723 & 6,37 & 95,63 & 16,17 & 8,86 & 100 & 100 \\
\hline Mittelwert & 97,0 & 35,13 & 0,45 & 395,15 & 0,16 & 0,49 & 4,99 & 160,54 & 15,25 & 10,77 & 99,44 & 86,36 \\
\hline Median & 98,4 & 32,02 & 0,48 & 388,00 & 0,15 & 0,46 & 5,27 & 137,60 & 14,09 & 8,86 & 100,00 & 100,00 \\
\hline SD & 44,4 & 12,45 & 0,13 & 103,67 & 0,04 & 0,26 & 1,33 & 107,52 & 6,53 & 5,46 & 1,69 & 16,86 \\
\hline Min & 30,4 & 17,50 & 0,22 & 255,28 & 0,10 & 0,17 & 3,28 & 48,92 & 8,29 & 4,80 & 94,93 & 60,04 \\
\hline Max & 157,3 & 52,21 & 0,58 & 575,80 & 0,24 & 0,96 & 7,21 & 327,76 & 25,90 & 19,72 & 100,00 & 100,00 \\
\hline
\end{tabular}

Tabelle 25: Pharmakokinetische Parameter der Intensivpatienten mit Nierenfunktionseinschränkung Teil 2 


\section{Danksagung}

Besonders danken möchte ich Dr. Lutz Binder für die Bereitstellung des interessanten Themas und die exzellente, unkomplizierte und freundschaftliche Betreuung zu jedem Zeitpunkt dieser Arbeit.

Ich danke Prof. Dr. Dr. Michael Oellerich für die Möglichkeit, in seiner Abteilung in ansprechender und professioneller Umgebung zu promovieren und für die konstruktive und schnelle Korrektur meines Manuskriptes.

Ich bedanke mich bei Annett Beckmann und Hagen Grüning für die gute Zusammenarbeit, sowie bei Dr. Miriam Puls für die Aufstellung der HPLC-Methode.

Weiterhin danke ich den Mitarbeitern der Abteilung der klinischen Chemie, sowie den Mitarbeitern der Stationen 0123 und 1026, die zum Gelingen dieser Arbeit beigetragen haben.

Ich danke meiner Frau Anika Hoppe für die moralische Unterstützung und das gewissenhafte Korrekturlesen meines Manuskriptes. 
